\title{
Lie Algebra Cohomology and the Fusion Rules
}

\author{
Constantin Teleman \\ Department of Mathematics, Harvard University, Cambridge, MA 02138, USA. \\ E-mail: teleman@zariski.harvard.edu
}

Received: 8 July 1994

\begin{abstract}
We prove a vanishing theorem for Lie algebra cohomology which constitutes a loop group analogue of Kostant's Lie algebra version of the Borel-Weil-Bott theorem. Consider a complex semi-simple Lie algebra $\mathfrak{g}_{\mathbb{C}}$ and an integrable, irreducible, negative energy representation $\mathscr{H}$ of $L \mathfrak{g}_{\mathbb{C}}$. Given $n$ distinct points $z_{k}$ in $\mathbb{C}$, with a finite-dimensional irreducible representation $V_{k}$ of $\mathfrak{g}_{\mathbb{C}}$ assigned to each, the Lie algebra $\mathfrak{g}_{\mathbb{C}[z]}$ of $\mathfrak{g}_{\mathbb{C}}$-valued polynomials acts on each $V_{k}$, via evaluation at $z_{k}$. Then, the relative Lie algebra cohomology $H^{*}\left(\mathfrak{g}_{\mathbb{C}[z]}, \mathfrak{g}_{\mathbb{C}} ; \mathscr{H} \otimes V_{1}\left(z_{1}\right) \otimes \cdots \otimes V_{n}\left(z_{n}\right)\right)$ is concentrated in one degree. As an application, based on an idea of G. Segal's, we prove that a certain "homolorphic induction" map from representations of $G$ to representations of $L G$ at a given level takes the ordinary tensor product into the fusion product. This result had been conjectured by $\mathrm{R}$. Bott.
\end{abstract}

\section{Contents}

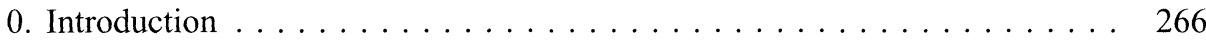

1. Preliminaries. . . . . . . . . . . . . . . . . . . . . . . . . . . 269

Sect. 1.1. The Borel-Weil-Bott Theorem. . . . . . . . . . . . . . . . 269

Sect. 1.2. Generalized BWB: The Case of Maximal Parabolic

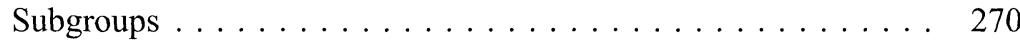

Sect. 1.3. Loop Groups . . . . . . . . . . . . . . . . . . . . . . . 271

Sect. 1.4. The Lie Algebra Statement . . . . . . . . . . . . . . . . . 272

Sect. 1.5. Fusion as Holomorphic Induction . . . . . . . . . . . . . 273

Sect. 1.6. Outline of the Proof of Theorem $0 \ldots \ldots \ldots \ldots 275$

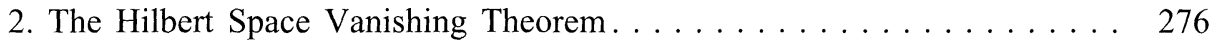

Sect. 2.0. Geometric Motivation . . . . . . . . . . . . . . . . 276

Sect. 2.1. The Setup . . . . . . . . . . . . . . . . . . . . . 277

Sect. 2.2. Statement of the Vanishing Theorem. . . . . . . . . . . . . 278

Sect. 2.3. Elementary Hilbert Space Properties . . . . . . . . . . . . . . . 279

Sect. 2.4. Nakano's Identity . . . . . . . . . . . . . . . . . . . . . . . 280

Sect. 2.5. Nakano Positivity . . . . . . . . . . . . . . . . . . . . . . 282

3. Lie Algebra Cohomology . . . . . . . . . . . . . . . . . . . . 284 
Sect. 3.1. The Formally Completed Representation . . . . . . . . . . . . . 284

Sect. 3.2. A Generalized BGG Resolution . . . . . . . . . . . . . . . . . 286

Sect. 3.3. Hilbert Space Representative . . . . . . . . . . . . . . . . . . 288

Sect. 3.4. The Knizhnik-Zamolodchikov Connection . . . . . . . . . . . . 291

Sect. 3.5. Bott Reflection, and End of Proof of Theorem 0 . . . . . . . . 293

Sect. 3.6. Application: The Fusion Rules . . . . . . . . . . . . . . . . 296 Appendixes

A. Lie Algebra Cohomology and the Hochschild- Serre Spectral Sequence. . . . . . . . . . . . . . . . . . . . . . . . . . 298

B. Two Useful Identities . . . . . . . . . . . . . . . . . . . . . . . . 300

C. Two Useful Inequalities. . . . . . . . . . . . . . . . . . . . . 308

References ......................... 310

\section{Introduction}

Let $G_{\mathbb{C}}$ be a complex semi-simple Lie group with compact form $G$, and let $B \subset G_{\mathbb{C}}$ be a Borel subgroup. The cohomology of $G_{\mathbb{C}}$-equivariant holomorphic line bundles over the flag manifold $G_{\mathbb{C}} / B$ of $G$ is given by the Borel-Weil-Bott theorem (BWB). Loosely speaking, the theorem asserts that, for most of these line bundles, the cohomology is concentrated in one degree, where it forms an irreducible representation of $G_{\mathbb{C}}$, whereas for a special class of line bundles it vanishes altogether. This statement extends to certain $G_{\mathbb{C}}$-equivariant holomorphic vector bundles over the generalized flag manifolds $G_{\mathbb{C}} / P$, corresponding to the parabolic subgroups $B \subset P \subset G_{\mathbb{C}}$. All such vector bundles are of the form $G_{\mathbb{C}} \times_{P} E$, for some holomorphic representation $E$ of $P$, and when $E$ is irreducible, the generalized version of BWB says, again, that the cohomology of this vector bundle lives (at most) in one degree and forms an irreducible representation of $G_{\mathbb{C}}$.

This paper is concerned with the problem of extending the BWB theorem to certain loop groups-specifically, the groups $L G$ and $L G_{\mathbb{C}}$ of smooth maps from the circle to a compact semi-simple ${ }^{1}$ Lie group $G$ and to its complexification $G_{\mathbb{C}}$. I must mention at the outset, however, that a proper construction of the cohomology of vector bundles over infinite-dimensional manifolds, such as are relevant to this problem, has been elusive so far, and in this direction, the present paper offers no contribution. Instead, the main result (Sect. 1.4. Theorem 0) is simply a statement about Lie algebra cohomology, an extension of Kostant's Lie algebra version [K] of the BWB theorem to the Lie algebra $L g_{\mathbb{C}}$ of $L G_{\mathbb{C}}$. Interestingly, this less ambitious theorem is powerful enough to solve one of the problems that motivated the whole enterprise: it determines the fusion rules for the WZW model for $G$, in a form that was conjectured by Bott. More precisely, we can show, following an argument of G. Segal's, that a naturally defined "holomorphic induction" map from the representations of $G$ to representations of $L G$ takes the ordinary tensor product into the fusion product.

Let us now return to the story. A BWB theory for loop groups was first proposed by $\mathrm{G}$. Segal $[\mathrm{P}-\mathrm{S}]$, in connection with their positive energy representations (PERs). Regarding the circle as the boundary of the unit disk $\Delta \subset \mathbb{C}$, the parabolic subgroup relevant here is the group $G_{\mathbb{C}}^{\Delta}$ of boundary-values of holomorphic maps from $\Delta$ to $G_{\mathbb{C}}$. (A Borel subgroup, which we shall make passing use of, is the

${ }^{1}$ I shall only treat the simply connected case. 
subgroup of loops in $G_{\mathbb{C}}^{A}$ mapping 0 into the Borel subgroup $B$ of $G_{\mathbb{C}}$.) We obtain a homomorphism from $G_{\mathbb{C}}^{\Delta}$ to $G_{\mathbb{C}}$ by evaluating holomorphic loops at the origin. Every $G_{\mathbb{C}}$-representation $E$ becomes in this way a representation of $G_{\mathbb{C}}^{4}$, and we can consider the associated vector bundle $\mathbf{E}:=L G \times_{G_{\mathbb{C}}^{A}} E$ over the flag manifold $X=L G_{\mathbb{C}} / G_{\mathbb{C}}^{\Delta}$. This vector bundle has no non-zero holomorphic sections, unless $E$ happens to contain the trivial representation, but if we twist $\mathbf{E}$ by sufficiently large power $\mathscr{L}^{\otimes h}$ of the fundamental line bundle ${ }^{2} . \mathscr{L}$ of $L G$, the resulting space of section is an irreducible PER $\mathscr{H}_{E, h}$ of $L G$ at level $h$ [P-S], whose space of lowest-energy states is a $G$-representation isomorphic to $E$. (We shall say that $\mathscr{H}_{E, h}$ is induced from $E$ at level $h$.) Moreover, we can obtain in this way all PERs of $L G$. This is a Borel-Weil theorem for $L G$. The "Bott part" of the theorem would additionally say that, in general, the cohomology $H^{*}\left(X ; \mathbf{E} \otimes \mathscr{L}^{\otimes h}\right)$ lives in at most one degree ${ }^{3} ;$ but while its moral truth is not in doubt, we cannot properly state it, for want of a good definition of higher cohomology. One must mention in this context that, if one is willing to replace $X$ by the thinner (but dense) algebraic variety $X^{\text {alg }}$, the union of the Schubert cells in $X$, the full BWB theorem has been proved by $\mathrm{Kumar}^{4}[\mathrm{Ku}]$.

This, however, is not the whole story, because the group $G_{\mathbb{C}}^{A}$ has some additional irreducible finite-dimensional representations. Choosing $n$ distinct points $\left\{z_{1}, \ldots, z_{n}\right\}$ inside $\Delta$, and assigning to each an irreducible representation $V_{k}$ of $G_{\mathbb{C}}$ leads to an irreducible representation of $G_{\mathbb{C}}^{\Delta}$ on $V_{1} \otimes \cdots \otimes V_{n}$, if we let a holomorphic loop act on each factor $V_{k}$ by means of its value at $z_{k}$. Denoting this representation by $V_{1}\left(z_{1}\right) \otimes \cdots \otimes V_{n}\left(z_{n}\right)$, or $\vec{V}(\vec{z})$, one may enquire about the space of holomorphic sections of the vector bundle $\overrightarrow{\mathbf{V}}(\vec{z}) \otimes \mathscr{L}^{\otimes h}$ over $X$.

The relevance of the space $H^{0}\left(X ; \overrightarrow{\mathbf{V}}(\vec{z}) \otimes \mathscr{L}^{\otimes h}\right)$, in connection with the Conformal Field theory notion of fusion, was pointed out by Segal. He observed that, if the representations $V_{k}$ are small enough to individually induce representations $\mathscr{H}_{k}:=\mathscr{H}_{V_{k}\left(z_{k}\right), h}$ of the loop group at level $h$, then the multiplicity of a given PER $\mathscr{H}$ in $H^{0}$ is naturally equal ${ }^{5}$ to its multiplicity in the fusion product of the $\mathscr{H}_{k}$ 's. Based on this, he conjectured that

(a) the space $H^{0}$ itself is the fusion product of the $\mathscr{H}_{k}$ 's. In particular, it is a PER, and a finite sum of irreducibles. By analogy with the BWB theorem, he also predicted that

(b) the higher cohomology $H^{q}\left(X ; \overrightarrow{\mathbf{V}}(\vec{z}) \otimes \mathscr{L}^{\otimes h}\right)$ vanishes.

In general, without any assumption on the $V_{k}$ 's, $H^{q}$ should vanish, if not altogether, in all but one degree.

As Segal observed, these two statements, if true, completely determine the fusion product. Let us namely consider, with Bott, the holomorphic induction map $i_{h}$ from $G_{\mathbb{C}}^{\Delta}$ to $L G_{\mathbb{C}}$ at level $h$. We shall postpone its actual definition until Sect. 1.5,

${ }^{2}$ Assuming that $G$ is simple. In this case, the condition is $\lambda_{E} \cdot \alpha_{\max } \leqq h$, where $\lambda_{E}$ is the highest weight of $E$ and $\alpha_{\max }$ the the highest root of $\mathfrak{g}$. In general, one must twist by powers of the basic line bundles corresponding to the simple factors of $G$.

3 The degree can be specified in terms of $h$ and the highest weight $\lambda_{E}$ of $E$; in particular, it is zero if $h$ is large enough.

${ }^{4}$ Kumar's version of BWB is not suited to deal with the issue of main concern in this paper, namely fusion. This is because on the algebraic flag variety $X^{a l y}$, there is no analogue of the vector bundles $\left(V_{1}\left(z_{1}\right) \otimes \cdots \otimes V_{n}\left(z_{n}\right)\right.$ we shall consider next.

5 The identity comes from a natural isomorphism of vector spaces. One of them is $\operatorname{Hom}_{L G}\left(\mathscr{H} ; H^{0}\right)$, but the other has not yet been defined (cf. Sect. 1.5 and Sect. 3.6). This leaves us with the rather dim-witted expression "naturally equal." 
but let us mention that $i_{h}$ extends the correspondence $E \rightarrow \mathscr{H}_{E, h}$, described earlier, assigning to any $G_{\mathbb{C}}$-representation $E$ the (expected) holomorphic Euler characteristic $\chi\left(X ; \mathbf{E} \otimes \mathscr{L}^{\otimes h}\right)$ of the bundle $\mathbf{E} \otimes \mathscr{L}^{\otimes h}$ - a virtual representation of $L G$ at level $h$. (By Kumar's previously quoted results, $i_{h}(E)$ is the Euler characteristic $\chi\left(X^{\text {alg }} ; \mathbf{E} \otimes \mathscr{L}^{\otimes h}\right)$. At any rate, when $E$ is irreducible, $i_{h}(E)$ is either a signed irreducible representation of $L G$ at level $h$, or 0 .) Based on Verlinde's prediction of the fusion coefficients [V], Bott conjectured that the map $i_{h}$ should take the ordinary tensor product of $G$-representations to the fusion product of their images.

Now if Segal's conjectures hold, then the fusion product $\mathscr{H}_{1} \otimes \cdots \otimes \mathscr{H}_{n}$ is the Euler characteristic $\chi\left(X ; \overrightarrow{\mathbf{V}}(\vec{z}) \otimes \mathscr{L}^{\otimes h}\right)$. We expect that

(c) unlike the cohomology groups, the Euler characteristic $\chi\left(X ; \overrightarrow{\mathbf{V}}(\vec{z}) \otimes \mathscr{L}^{\otimes h}\right)$ does not change when the points $z_{k}$ are all moved to the origin.

After all, we are continuously deforming the holomorphic structure of the bundle. But $\chi\left(X ; \overrightarrow{\mathbf{V}}(\overrightarrow{0}) \otimes \mathscr{L}^{\otimes h}\right)$ is what we previously called $\chi\left(X ; \mathbf{V}_{1} \otimes \cdots \otimes \mathbf{V}_{n} \otimes \mathscr{L}^{\otimes h}\right)$, which is by "definition" $i_{h}\left(V_{1} \otimes \cdots \otimes V_{n}\right)$. Hence the conclusion

(d) the fusion product $\mathscr{H}_{1} \otimes \cdots \otimes \mathscr{H}_{n}$ is $i_{h}\left(V_{1} \otimes \cdots \otimes V_{n}\right)$.

In this form, Segal's conjectures are still open, and I shall not address them here. Instead, this paper is based on the simple observation (Sect. 1.5, Sect. 3.6) that Segal's argument for determining fusion can be rewritten purely in terms of Lie algebra cohomology. This is somewhat analogous to the fact that the usual BWB theorem is equivalent to Kostant's Lie algebra cohomology reformulation. Let us be more precise, and consider the space $\Gamma_{h}$ of smooth sections of $\mathscr{L}^{\otimes h}$ over $L G$, on which $\mathfrak{g}_{\mathbb{C}}^{\Delta}$ acts by right translation. The Dolbeault complex for the holomorphic vector bundle $\overrightarrow{\mathbf{V}}(\vec{z}) \otimes \mathscr{L}^{\otimes h}$ over $X=L G_{\mathbb{C}} / G_{\mathbb{C}}^{4}$ (which, as a smooth manifold, is isomorphic to $L G / G$ ) is naturally isomorphic to the standard complex of relative Lie algebra cohomology of the pair $\left(\mathfrak{g}_{\mathbb{C}}^{4}, \mathfrak{g}_{\mathbb{C}}\right)$ with coefficients in $\Gamma_{h} \otimes \vec{V}(\vec{z})$

$$
\mathscr{C}^{*}\left(\mathfrak{g}_{\mathbb{C}}^{\Delta}, \mathfrak{g}_{\mathbb{C}} ; \Gamma_{h} \otimes \vec{V}(\vec{z})\right):=\operatorname{Hom}_{\mathfrak{g}_{\mathbb{C}}}\left(\Lambda^{*}\left(\mathfrak{g}_{\mathbb{C}}^{\Delta} / \mathfrak{g}_{\mathbb{C}}\right) ; \Gamma_{h} \otimes \vec{V}(\vec{z})\right) .
$$

If we had a "Peter-Weyl" theorem for $L G$ and could factor the $L G \times L G$-space $\Gamma_{h}$ as (a completion of) $\bigoplus_{\mathscr{H}} \mathscr{H} \otimes \mathscr{H}^{t}$, with the sum ranging over all PERs $\mathscr{H}$ at level $h$, it would follow that

$$
H^{q}\left(X ; \vec{V}(\vec{z}) \otimes \mathscr{L}^{\otimes h}\right)=\bigoplus_{\mathscr{H}} \mathscr{H} \otimes H^{q}\left(\mathfrak{g}_{\mathbb{C}}^{4}, \mathfrak{g}_{\mathbb{C}} ; \vec{V}(\vec{z}) \otimes \mathscr{H}^{t}\right)
$$

This would prove conjecture (a). Unfortunately, no applicable version of the PeterWeyl statement seems to be true, and the above identity remains conjectural. However, we can prove the following:

(1) If one replaces $H^{q}\left(X ; \vec{V}(\vec{z}) \otimes \mathscr{L}^{\otimes h}\right)$ with the space $\oplus \mathscr{H} \mathscr{H} \otimes H^{q}$ $\left(\mathfrak{g}_{\mathbb{C}}^{\Delta}, \mathfrak{g}_{\mathbb{C}} ; \vec{V}(\vec{z}) \otimes \mathscr{H}^{t}\right)$, Segal's conjectures are true.

(2) This is sufficient to prove that the map $i_{h}$ takes tensor products to fusion products.

The idea of applying Lie algebra cohomology techniques to the study of fusion is not new; such methods have been successfully used by Feigin and Fuchs, who in [F-F] determined the fusion rules for $\mathfrak{g}=\mathfrak{s l}_{2}$. But while their methods are, generally speaking, more powerful than our custom-made approach, the form of their output is less convenient, and it is unclear whether the higher-rank case could be handled with comparable ease.

Shortly after this paper was completed, an article of G. Faltings appeared [F2], in which the fusion rules for all classical groups and for $G_{2}$ were determined by 
a somewhat bare-handed approach (but using results of Tsuchiya and Kanie [T-K], and a "factorization theorem," needed to prove associativity of the fusion product). The method of this paper is preferable, in the author's opinion, not only because it works for all groups, but also because no calculation is needed to find the answer (as can be seen from the five-line proof of Theorem 1 in Sect. 3.6). Associativity of fusion also follows without any appeal to the "factorization theorem." True, the technicalities needed to justify the steps corresponding to (a)-(d) are less pleasant than the more elementary calculations of [F2], but they are related to the difficulties of analysis on infinite-dimensional manifolds, and as such they are of some interest in their own right. It should be mentioned, as this may not be evident, that in the background of the proof is the geometry and analysis of the flag manifold $L G / G$.

I could not close this introduction without gratefully acknowledging some of the numerous people I have fallen indebted to, mathematically or otherwise. Topping the list are my co-advisors, Professor Graeme Segal and Professor Raoul Bott; this work would not have come about without their support and mathematical insight. It is fair to say that most things worth remembering in the present paper are due to them. Any reader familiar with Segal's ideas on CFT will recognize his handprint in the entire setup. Next in line is Professor Cliff Taubes, whose thoughts about the geometry and analysis on loop groups gave me the first incentives to study the topic. Numerous helpful suggestions were made by Dr. Antony Wassermann (Cambridge). I must also thank Edward Frenkel for providing helpful answers and references, Andras Szenes and Richard Wentworth for some helpful discussions, and Sheldon Chang for raising a few important points.

This paper is essentially my Ph.D. thesis (Harvard, 1994). A portion of it (essentially, Sect. 2) was written as an Master's thesis at Cambridge University. This was supported by a Herschel Smith Fellowship and an ORS award.

\section{Preliminaries}

1.1. The Borel-Weil-Bott Theorem. Let $\mathfrak{g}$ be the Lie algebra of the compact, simple, simply connected Lie group $G$ and let $\mathrm{t} \subset \mathfrak{g}$ be a Cartan subalgebra. Choose a positive root system $\Phi$, let $n \subset \mathfrak{g}_{\mathbb{C}}$ be the maximal nilpotent subalgebra spanned by the root vectors $\left\{e_{-\alpha} \mid \alpha \in \Phi\right\}$ and $B \subset G_{\mathbb{C}}$ the Borel subgroup with Lie algebra $\mathfrak{b}=\mathfrak{n} \tilde{\oplus} \mathrm{t}$. We shall make use of the basic inner product on $\mathfrak{g}$, the unique adinvariant inner product in which the highest root $\alpha_{\max }$ has square-length $2 ;\left\{\xi_{a}\right\}$ will denote an orthonormal basis of $\mathfrak{g}$ in this inner product. Recall that a weight of $\mathfrak{g}$-a character of the maximal torus $T$-is called singular if it is orthogonal to one of the positive roots, regular otherwise, dominant if all inner products with the positive roots are non-negative. A distinguished dominant regular weight is $\rho$, the half-sum of the positive roots. Holomorphic $G_{\mathbb{C}}$-equivariant line bundles over the flag variety $Y:=G_{\mathbb{C}} / B$ are all of the form $L_{\lambda}=G_{\mathbb{C}} \times_{B} \mathbb{C}_{\lambda}$, corresponding to holomorphic characters $\lambda$ of $B$, thus to weights of $\mathfrak{g}$. For instance, the canonical bundle $K_{Y}$ corresponds to $(-2 \rho)$. Denote by $\ell(\lambda)$ the number of positive roots having a negative inner product with the regular weight $\lambda^{6}{ }^{6}$

\footnotetext{
$6 \ell(\lambda)$ is also the length of the unique Weyl group element taking $\lambda$ to the positive Weyl chamber. Recall that the simple Weyl reflections $s_{k}$-reflections about the hyperplanes orthogonal to the simple roots $\alpha_{k}$-generate the action of the Weyl group of $G$ on $\mathrm{t}$, and that the Weyl transforms of the positive chamber are disjoint and cover all the regular weights. The length of a Weyl group element is the length of its shortest expression as a product of simple reflections.
} 
The cohomology of the $L_{\lambda}$ 's is given by the Borel-Weil-Bott theorem:

\section{Theorem. ([B])}

(a) If the shifted weight $(\lambda+\rho)$ is regular, then $H^{q}\left(Y ; L_{\lambda}\right)=0$, except for $q=\ell(\lambda+\rho)$. In that case, $H^{q}$ is the irreducible representation of $G$ whose $\rho$ shifted highest weight is in the Weyl orbit of $(\lambda+\rho)$;

(b) If $(\lambda+\rho)$ is singular, then $H^{q}\left(Y ; L_{\lambda}\right)=0$ for all $q$.

1.2. Generalized BWB: The Case of Maximal Parabolic Subgroups. Our approach to the loop group theory is modeled on the BWB theorem for the generalized flag manifolds $Y_{P}=G_{\mathbb{C}} / P$ corresponding to parabolic subgroups $P$ of $G_{\mathbb{C}}$. Recall that the generalized BWB theorem asserts that the cohomology $H^{*}\left(Y_{P} ; \mathbf{E}\right)$ lives in only one degree, if the holomorphic bundle $\mathbf{E}$ comes from an irreducible representation $E$ of $P$. Of special interest for loop groups is the case of maximal parabolic subgroups ${ }^{7}$ and vanishing higher cohomology, which we now take a closer look at.

A parabolic subgroup $P$ factors as a semidirect product $R_{\mathbb{C}} \tilde{\times} U$ of a complex reductive subgroup $R_{\mathbb{C}}$ and a unipotent $U$. If $E$ is irreducible, $U$ acts trivially, and so $E$ is an irreducible representation of $R_{\mathbb{C}}$. It can, therefore, be realized as the space of holomorphic sections of an equivariant line bundle $L_{\lambda}$ over the flag manifold $R_{\mathbb{C}} /\left(B \cap R_{\mathbb{C}}\right) \cong P / B$ of the semi-simple part of $R_{\mathbb{C}}$, for some dominant character $\lambda$ of $B$. The higher cohomology of $L_{\lambda}$ over $P / B$ vanishes, by the usual BWB theorem. By considering the holomorphic fibration

$$
\begin{aligned}
P / B \rightarrow G_{\mathbb{C}} / B & =Y \\
\downarrow & \\
G_{\mathbb{C}} / P & =Y_{P}
\end{aligned}
$$

we can see that $H^{*}\left(Y_{P} ; \mathbf{E}\right) \cong H^{*}\left(\Upsilon ; L_{\lambda}\right)$. The BWB theorem for $Y$ gives

Proposition 1.2.1. The bundle $\mathbf{E}$ has holomorphic sections if and only if $\lambda$ is dominant.

Its higher cohomology vanishes if and only if $(\lambda+\rho)$ is dominant.

Splitting the Lie algebra $\mathfrak{r}=\mathfrak{g}^{\prime} \oplus \mathfrak{I}$ of $R$ into the semi-simple and abelian parts decomposes the weight $\lambda$ as $\lambda^{\prime}+\lambda^{\perp}$, where $\lambda^{\prime}$ is the highest weight of $E$ for $\mathrm{g}^{\prime}$, and $\lambda^{\perp}$ is the character by which $\mathbb{I}$ acts. When $P$ is maximal parabolic, $\mathbb{I}$ is 1 -dimensional and $\mathfrak{g}^{\prime}$ has rank exactly one less than $\mathfrak{g}$. More precisely, the simple roots $\alpha_{1}, \ldots, \alpha_{\ell-1}$ of $\mathfrak{g}^{\prime}$ are all the simple roots of $\mathfrak{g}$, save one. The missing root $\alpha_{f}$ does not lie in $\mathfrak{l}$, except in the uninteresting case when $\mathfrak{g}^{\prime}$ is a summand in $\mathfrak{g}$, but rather projects to a non-zero antidominant weight $(-\mu)$ of $\mathfrak{g}^{\prime}$ (the inner products $\alpha_{k} \cdot \alpha_{f}$ are non-positive).

Let $\beta \in \mathbb{I}$ be the element for which $\beta \cdot \alpha_{f}=1$ in the basic inner product; it is the smallest integral character of $\mathfrak{I} \subset \mathrm{t}$. Call the integer $h=\lambda^{\perp} / \beta$ the level of $E$. We want to express, in terms of $\lambda^{\prime}$ and $h$, the condition that $(\lambda+\rho)$ is dominant. Note first that

$$
\lambda \text { is dominant } \Leftrightarrow \lambda^{\prime} \text { is dominant, and } \lambda^{\prime} \cdot \mu \leqq h \text {. }
$$

This translates the conditions $\lambda \cdot \alpha_{k} \geqq 0$ and $\lambda \cdot \alpha_{\ell} \geqq 0$. Further, let $\rho^{\prime}$ be the halfsum of the positive roots of $\mathfrak{g}^{\prime}$. Interestingly enough, $\rho^{\prime}$ is the projection of $\rho$ on

\footnotetext{
${ }^{7}$ Note that $G_{\mathbb{C}}^{\Delta}$ is a maximal parabolic subgroup of $L G_{\mathbb{C}}$.
} 
$\mathrm{g}^{\prime}$, and so we may set $\rho-\rho^{\prime}=c \cdot \beta$ for some number $c$. We can reformulate $(1.2 .1)$ as

Proposition 1.2.2. The bundle $\mathbf{E}$ has holomorphic sections iff $\lambda^{\prime} \cdot \mu \leqq h$. Its higher cohomology vanishes iff $\left(\lambda^{\prime}+\rho^{\prime}\right) \cdot \mu \leqq h+c$.

It follows in particular, since $\mu$ is dominant, that only finitely many E's will have holomorphic sections at a given level $h$.

1.3. Loop Groups. A representation of $L G$ is of positive energy if it admits a continuous action of $S^{1}$, intertwining with the rotation of the loops, which decomposes it into finite-dimensional eigenspaces; we require that only positive characters occur. The associated infinitesimal action is called the energy. One should recall [P-S] that all non-trivial PERs of a loop group are projective, that is, representations of a central extension of the loop group. They are also completely reducible, and the irreducibles among them are representations of smooth $S^{1}$-extensions of $L G$-respectively, holomorphic $\mathbb{C}^{\times}$-extensions of $L G_{\mathbb{C}}$. If $G$ is simply connected, as we shall henceforth assume, $L G$ and $L G_{\mathbb{C}}$ admit universal smooth (resp. holomorphic) central extensions $\tilde{L} G$ and $\tilde{L} G_{\mathbb{C}}$ by a (complex) torus whose rank is equal to the number of simple factors in $G$. In particular, when $G$ is simple ${ }^{8}$, the projective cocycle of an irreducible PER is determined by its level, an integral character $h \in \mathbf{Z}$ of the centre $\mathbb{C}^{\times}$of $\tilde{L} G_{\mathbb{C}}$. In addition, for one of the choices of the universal extension, only positive values of $h$ can occur. The smooth (resp. holomorphic) line bundle $\mathscr{L}$ over $L G$ (resp. $L G_{\mathbb{C}}$ ) associated to this universal central extension is called the fundamental line bundle.

Definitions and Notation. Write $c$ for the dual Coxeter number $\left(\rho \cdot \alpha_{\max }+1\right)$ of $\mathfrak{g}$. Having fixed the level $h$, there is an " $(h+c)$-action" of the affine Weyl group $W_{\text {aff }}$ on the Cartan subalgebra $\mathrm{t} \subset \mathrm{g}$. (We expect from the finite dimensional case that a shifted action of $W_{\text {aff }}$ should be more relevant than the natural one, and this is indeed the case. To avoid cumbersome wording later, we shall incorporate the shifts in the present definitions.) This action is generated by the simple Weyl reflections $s_{1}, \ldots, s_{\text {, }}$ about the hyperplanes orthogonal to the simple roots, and by the reflection $s_{t+1}$ about the affine hyperplane $\left\{\tau \in \mathrm{t} \mid \alpha_{\max }(\tau)=h+c\right\}$. These $\ell+1$ hyperplanes bound a simplex in $\mathrm{t}$, the positive Weyl alcove, whose $W_{\text {aff }}$-transforms, the Weyl alcoves, form a tiling of t. $W_{\text {aff }}$ acts simply transitively on the set of alcoves.

We shall call an irreducible representation $V$ of $G$

- regular, if its $\rho$-shifted highest weight $\left(\lambda_{V}+\rho\right)$ falls inside some Weyl alcove,

- singular otherwise;

- antidominant, if $\left(\lambda_{V}+\rho\right)$ falls within, or on the boundary of the positive Weyl alcove.

The length $\ell(V)$ of $V$ is the least number of reflections needed to bring the $\rho$ shifted highest weight $\left(\lambda_{V}+\rho\right)$ in the closed positive Weyl alcove. Thus $\ell(V)=0$ if $V$ is antidominant ${ }^{9}$. Two representations $V$ and $W$ shall be called affine-conjugate at level $h$ if their $\rho$-shifted highest weights are in the same orbit of the $(h+c)$-action of $W_{\text {aff. }}$.

\footnotetext{
8 The latter is not a restriction, since a simply connected semi-simple group is a product of simple factors. The non-simply connected case requires more discussion, though.

9 The length is truly relevant only if $V$ is regular; in that case, $\ell(V)$ is also the length of the unique affine Weyl group element bringing $(\lambda+\rho)$ within the positive alcove.
} 
Remark. The length of a representation and its affine-conjugacy class do not depend on the choice of the positive root system.

1.4. The Lie Algebra Statement. Let $\mathfrak{g}_{\mathbb{C}[z]}$ be the Lie algebra of $\mathfrak{g}_{\mathbb{C}}$-valued polynomials. Given $n$ points $z_{1}, \ldots, z_{n} \in \mathbb{C}$, with a representation $V_{k}$ of $G$ assigned to each, $\mathfrak{g}_{\mathbb{C}[z]}$ acts on the tensor product $V_{1} \otimes \cdots \otimes V_{n}$ by means of the evaluations at the points $z_{k}$. As before, we denote the resulting representation by $V_{1}\left(z_{1}\right) \otimes \cdots \otimes V_{n}\left(z_{n}\right)$, or $\vec{V}(\vec{z})$. If the factors $V_{k}$ are irreducible, then so is $\vec{V}(\vec{z})$.

Pick an irreducible negative energy representation $\mathscr{H}$ of $L \mathfrak{g}$ at level $(-h)$, and let $\hat{\mathscr{H}}$ denote its formal completion with respect to the energy grading (the direct product of the energy eigenspaces). The central extension of $L \mathfrak{g}$ can be uniquely trivialized over $\mathfrak{g}_{\mathbb{C}[z]}$, and thus $\hat{\mathscr{H}}$ is a genuine representation of $\mathfrak{g}_{\mathfrak{C}[z]}$ (though not of its "thicker" version $\mathfrak{g}_{\mathbb{C}}^{A}=\operatorname{Lie}\left(G_{\mathbb{C}}^{\Delta}\right)$ ). It has the important property that it splits as a direct product of its $\mathfrak{g}$-isotypical components, all of which are of finite-dimensional type (cf. Sect. 3.1), and one can easily see that this property is shared by $\hat{\mathscr{H}} \otimes \vec{V}(\vec{z})$. Denote by $\mathscr{C}\left(\mathfrak{g}_{\mathbb{C}[z]}, G\right)$ the category of complex representations of $\mathfrak{g}_{\mathbb{C}[z]}$ satisfying this condition. It is an abelian category with enough injectives, and the functor of $\mathfrak{g}_{\mathbb{C}[z]}$-invariants is left exact on it (cf. Appendix A). The corresponding right derived functors, applied to an object $M$, are the relative Lie algebra cohomology groups $H^{*}\left(\mathfrak{g}_{\mathbb{C}[z]}, \mathfrak{g} ; M\right)$ of the pair $\left(\mathfrak{g}_{\mathbb{C}[z]}, \mathfrak{g}\right)$ with coefficients in $M$. The main theorem of this paper determines this cohomology for $M=\hat{\mathscr{H}} \otimes \vec{V}(\vec{z})$, after one final bit of notation. Set

$$
h^{q}(M):=\operatorname{dim} H^{q}(\ldots), \quad \chi(M)=\sum_{q}(-1)^{q} \cdot h^{q}(M) .
$$

The characteristic $\chi$ is defined only if the $h^{q}$ are finite and vanish for large $q$. Finally, let $E^{\mathrm{t}}$ be the $G$-representation dual to the space of highest-energy states in $\mathscr{H}$.

Theorem 0. Assume that the points $z_{k}$ are distinct and the representations $V_{k}$ are irreducible. Then, the relative Lie algebra cohomology $H^{*}\left(\mathfrak{g}_{\mathbb{C}[z]}, \mathfrak{g} ; \hat{\mathscr{H}} \otimes\right.$. $\vec{V}(\vec{z}))$ is always finite-dimensional, and lives at most in one degree. Specifically,

(a) If $n=1$, then $h^{q}=0$ for all $q$, unless $V_{1}$ and $E^{t}$ are affine-conjugate at level $h$. In that case,

$$
h^{q}\left(\hat{\mathscr{H}} \otimes V_{1}\left(z_{1}\right)\right)=\left\{\begin{array}{ll}
1, & \text { for } q=\ell\left(V_{1}\right) \\
0 & \text { otherwise. }
\end{array} .\right.
$$

(Note that this can only happen if $V_{1}$ is regular.)

(b) If all the $V_{k}^{\prime} s$ are regular at level $h$, then $h^{q}(\hat{\mathscr{H}} \otimes \vec{V}(\vec{z}))=0$ for all $q$, except possibly for $q=\sum_{k} \ell\left(V_{k}\right)$. It follows, for that value of $q$, that $h^{q}(\hat{\mathscr{H}} \otimes \vec{V}(\vec{z}))=(-1)^{q} \cdot \chi(\hat{\mathscr{H}} \otimes \vec{V}(\vec{z}))$;

$\left(\mathrm{b}^{\prime}\right)$ The Euler characteristic remains unchanged when all the points coalesce: $\chi(\hat{\mathscr{H}} \otimes \vec{V}(\vec{z}))=\chi(\hat{\mathscr{H}} \otimes \vec{V}(\overrightarrow{0}))$. The latter can be computed from (a).

(c) In particular, when all the $V_{k}$ 's are antidominant regular at level $h$, then

$$
\begin{aligned}
H^{q}(\hat{\mathscr{H}} \otimes \vec{V}(\vec{z})) & =0 \text { for } q>0, \text { and } \operatorname{dim}(\hat{\mathscr{H}} \otimes \vec{V}(\vec{z}))^{\mathfrak{g}_{\mathbb{C}[z]}} \\
& =\chi\left(\hat{\mathscr{H}} \otimes\left(V_{1} \otimes \cdots \otimes V_{n}\right)(0)\right) .
\end{aligned}
$$

(d) Finally, when one of the $V_{k}$ 's is singular, then $h^{q}=0$ for all $q$. 
The proof of Theorem 0 occupies most of this paper. Its relevance lies in the connection between the space $H^{0}$ and the fusion operation, motivated by Conformal Field Theory, which we now define.

1.5. Fusion as Holomorphic induction. Let $\Delta_{1}, \ldots, \Delta_{n}$ be a collection of nonoverlapping disks inside $\Delta$, centered at the points $z_{k}$, with parametrised boundaries $S_{1}, \ldots, S_{n}$. Let $\Sigma=\Delta \backslash\left(\Delta_{1} \cup \cdots \cup \Delta_{n}\right)$, and $G_{\mathbb{C}}^{\Sigma}$ be the group of holomorphic maps from $\Sigma$ to $G_{\mathbb{C}}$, with smooth boundary-values. To each $S_{k}$, we assign a PER $\mathscr{H}_{k}$ of the loop group $L_{k} G:=\operatorname{Map}\left(S_{k} ; G\right)$. ( $L G$ will continue to denote the group $\operatorname{Map}(S ; G)$.) The tensor product $\left(\mathscr{H}_{1}\right)^{t} \otimes \cdots \otimes\left(\mathscr{H}_{n}\right)^{t}$ of their duals is a projective representation of $G_{\mathbb{C}}^{\Sigma}$. Given a PER $\mathscr{H}$ of $L G$, we are interested in the space of $G_{\mathbb{C}}^{\Sigma}$-equivariant homomorphisms

$$
M\left(\mathscr{H} \mid \mathscr{H}_{1}, \ldots, \mathscr{H}_{n}\right):=\operatorname{Hom}_{G_{\mathbb{C}}^{\Sigma}}\left(\left(\mathscr{H}_{1}\right)^{t} \otimes \cdots \otimes\left(\mathscr{H}_{n}\right)^{t} ; \mathscr{H}^{t}\right) .
$$

The question makes sense, because $\operatorname{Hom}(\cdots)$ is a genuine representation of $G_{\mathbb{C}}^{\Sigma}$. Indeed, at the Lie algebra level, the projective cocycle can be written as the boundary integral

$$
h \cdot \omega(\xi, \eta)=\frac{h}{2 \pi} \cdot \int_{\partial \Delta} \xi \cdot d \eta+\sum_{k} \frac{h}{2 \pi} \cdot \int_{S_{k}} \xi \cdot d \eta=\frac{h}{2 \pi} \cdot \int_{\partial \Sigma} \xi \cdot d \eta,
$$

and if $\xi$ and $\eta$ are holomorphic, this vanishes by Stokes' theorem. For the precise definition of $M(\cdot \mid \cdot)$, the topologies on the $\mathscr{H}_{k}$ 's must be specified. Several natural choices lead to the same spaces $M$, and we shall settle in the end for the algebraic definition (Sect. 3.6), commonly used for its simplicity; but for the moment, we make no restrictions.

The spaces (1.5.1) are the conformal blocks of the field theory associated to the loop group $L G$ at level $h$. Their knowledge can be used to predict the "physical" evolution along the space-time $\Sigma$, in which the $n$ worlds $S_{1}, \ldots, S_{n}$ "fuse together" to the space $S$. This motivates the following definition of the fusion of the representations $\mathscr{H}_{k}$ along $\Sigma$ :

$$
\Sigma\left(\mathscr{H}_{1}, \ldots, \mathscr{H}_{n}\right)=\bigoplus_{\mathscr{H}} \mathscr{H} \otimes \operatorname{Hom}_{G_{\mathbb{C}}^{\Sigma}}\left(\left(\mathscr{H}_{1}\right)^{t} \otimes \cdots \otimes\left(\mathscr{H}_{n}\right)^{t} ; \mathscr{H}^{t}\right) .
$$

The sum is taken over all irreducible PERs $\mathscr{H}$ of $L G$ at level $h$. Note that

(i) $\Sigma\left(\mathscr{H}_{1}, \ldots, \mathscr{H}_{n}\right)$ is a PER of $L G$, and that

(ii) there exists a $G_{\mathbb{C}}^{\Sigma}$-equivariant map from $\Sigma\left(\mathscr{H}_{1}, \ldots, \mathscr{H}_{n}\right)$ to the completed tensor product $\mathscr{H}_{1} \hat{\otimes} \cdots \hat{\otimes} \mathscr{H}_{n}$. (Just take the trace over $\mathscr{H}$.)

Moreover, $\Sigma(\ldots)$ is universal with these properties. In a sense, we could say that it is the positive energy part of $\mathscr{H}_{1} \hat{\otimes} \cdots \hat{\otimes} \mathscr{H}_{n}$.

The spaces $M(\cdot \mid \cdot)$ have a very rich structure [S], but we shall only concentrate on a very crude invariant, namely their dimensions ${ }^{10}$. Accordingly, let $A_{h}$ be the free abelian group based on the set $B_{h}$ of PERs of $L G$ at level $h$. The $n$-ary fusion product is the $n$-linear map from $A_{h} \times \cdots \times A_{h}$ to $A_{h}$ defined by

$$
\circledast\left(\mathscr{H}_{1}, \ldots, \mathscr{H}_{n}\right):=\bigoplus_{\mathscr{H} \in B_{h}} \mathscr{H} \cdot \operatorname{dim} M\left(\mathscr{H} \mid \mathscr{H}_{1}, \ldots, \mathscr{H}_{n}\right) .
$$

10 They turn out to be finite-dimensional 
The idea of encoding the dimensions of the $M$ 's into this multiplicative structure is due to E. Verlinde [V], and $A_{k}$, endowed with the binary fusion product, is also known as the Verlinde algebra. It turns out that the multiplication is easily described. Following Bott [B2], define the "holomorphic induction" map from representations of $G$ to PERs of $L G$ at level $h$ by

$$
\begin{aligned}
i_{h}(V) & =(-1)^{\ell(W)} \mathscr{H}_{W} & & \text { if } V \text { is regular } \\
& =0 & & \text { if } V \text { is singular, }
\end{aligned}
$$

where, in the regular case, $W$ is the unique antidominant affine-conjugate of $V$. As an application of Theorem 0 , we shall prove

Theorem 1. Bott's holomorphic induction map takes the tensor product in $R(G)$ into the fusion product on $A_{h}$ :

$$
\circledast\left(i_{h}\left(V_{1}\right), \ldots, i_{h}\left(V_{n}\right)\right)=i_{h}\left(V_{1} \otimes \cdots \otimes V_{n}\right) .
$$

The proof is based on an argument of G. Segal's and the complete account is given in Sect. 3.6. However, before closing this section, we would like to mention the motivation for realising of fusion as a space of holomorphic sections (Conjecture (a) of the Introduction).

Segal's basic assumption is that, in the above universal property-definition of the fusion product, the positive energy condition (i) is superfluous ${ }^{11}$. In other words, $\Sigma\left(\mathscr{H}_{1}, \ldots, \mathscr{H}_{n}\right)$ can be simply defined as the $L G$-representation satisfying, for any representation $\mathscr{H}$ of $L G$, not necessarily of positive energy,

$$
\operatorname{Hom}_{L G_{\mathbb{C}}}\left(\mathscr{H} ; \Sigma\left(\mathscr{H}_{1}, \ldots, \mathscr{H}_{n}\right)\right) \cong \operatorname{Hom}_{G_{\mathbb{C}}^{\Sigma}}\left(\mathscr{H} ; \mathscr{H}_{1} \hat{\otimes} \cdots \hat{\otimes} \mathscr{H}_{n}\right)
$$

Now the spaces $\mathscr{H}_{k}$ can be individually realised as spaces of holomorphic sections of $\mathscr{L}^{\otimes h} \otimes \mathbf{V}_{k}\left(z_{k}\right)$ over the flag varieties $L_{k} G_{\mathbb{C}} / G_{\mathbb{C}}^{\Delta_{k}}$. In somewhat dishonest, but self-explanatory notation, we shall write this as

$$
\mathscr{H}_{k}=\operatorname{Ind}_{G_{\mathbb{C}}^{u_{k}}}^{L_{k} G_{\mathbb{C}}}\left(V_{k}\left(z_{k}\right)\right) .
$$

Correspondingly, the completed tensor product is realised as

$$
\mathscr{H}_{1} \hat{\otimes} \cdots \hat{\otimes} \mathscr{H}_{n}=\operatorname{Ind}_{G_{\mathbb{C}}^{\Delta_{1}} \times \cdots \times G_{\mathbb{C}}^{\Delta_{n}}}^{L_{L_{1}} G_{\mathbb{C}} \times \cdots \times L_{n} G_{\mathbb{C}}}\left(V_{1}\left(z_{1}\right) \otimes \cdots \otimes V_{n}\left(z_{n}\right)\right) .
$$

Segal observed (cf. 3.6.8) that the inclusion $G_{\mathbb{C}}^{\Sigma} / G_{\mathbb{C}}^{\Delta} \subset \prod_{k} L_{k} G_{\mathbb{C}} / G_{\mathbb{C}}^{\Delta_{k}}$ of $G_{\mathbb{C}^{-}}^{\Sigma}$ homogeneous spaces is really an isomorphism. Because of this, (1.5.6) becomes

$$
\mathscr{H}_{1} \hat{\otimes} \cdots \hat{\otimes} \mathscr{H}_{n}=\operatorname{Ind}_{G_{\mathbb{C}}^{\Delta}}^{G^{\Sigma}}\left(V_{1}\left(z_{1}\right) \otimes \cdots \otimes V_{n}\left(z_{n}\right)\right)
$$

\footnotetext{
11 Dropping assumption (i) is closely related to associativity of fusion. If positivity needs not be verified, an abstract nonsense argument allows one to factor the fusion product through any closed loop between $S$ and some of the $S_{k}$ 's.
} 
and by Frobenius reciprocity,

$$
\begin{aligned}
\operatorname{Hom}_{G_{\mathbb{C}}^{\Sigma}}\left(\mathscr{H} ; \mathscr{H}_{1} \hat{\otimes} \cdots \hat{\otimes} \mathscr{H}_{n}\right) & \cong \operatorname{Hom}_{G_{\mathbb{C}}^{\Sigma}}\left(\mathscr{H} ; \operatorname{Ind}_{G_{\mathbb{C}}^{\Delta}}^{G_{\mathbb{C}}^{\Sigma}}\left(V_{1}\left(z_{1}\right) \otimes \cdots \otimes V_{n}\left(z_{n}\right)\right)\right) \\
& \cong \operatorname{Hom}_{G_{\mathbb{C}}^{\Delta}}\left(\mathscr{H} ; V_{1}\left(z_{1}\right) \otimes \cdots \otimes V_{n}\left(z_{n}\right)\right) .
\end{aligned}
$$

But by Frobenius reciprocity again

$$
\begin{aligned}
& \operatorname{Hom}_{G_{\mathbb{C}}^{\Sigma}}\left(\mathscr{H} ; V_{1}\left(z_{1}\right) \otimes \cdots \otimes V_{n}\left(z_{n}\right)\right) \\
& \quad \cong \operatorname{Hom}_{L G_{\mathbb{C}}}\left(\mathscr{H} ; \operatorname{Ind}_{G_{\mathbb{C}}^{\Delta}}^{L G_{\mathbb{C}}}\left(V_{1}\left(z_{1}\right) \otimes \cdots \otimes V_{n}\left(z_{n}\right)\right)\right),
\end{aligned}
$$

whence by definition, using our earlier notation, we would obtain

$$
\Sigma\left(\mathscr{H}_{1}, \ldots, \mathscr{H}_{n}\right) \cong H^{0}\left(X ; \mathscr{L}^{\otimes h} \otimes \overrightarrow{\mathbf{V}}(\vec{z})\right)
$$

The expendability of the positive energy condition (i) in this approach to fusion is an open question. But what we can and will extract from this is Eq. (1.5.8), which will allow us in Sect. 3.6 to find fusion as a space of $\mathfrak{g}_{\mathbb{C}}^{A}$-invariants.

1.6. Outline of the Proof of Theorem 0. Even though the strategy is simple, the detailed proof of Theorem 0 is quite long, and a brief outline containing the noteworthy ideas should be welcome. The reader willing to believe all of the statements in this section should be cautioned that the remainder of the paper, with the likely exception of Sect. 3.6, where fusion is discussed, will be of little benefit.

The argument roughly follows the original proof of BWB. First, we establish the antidominant case (c), using a positivity argument in a Hilbert space version of the Lie algebra cohomology complex. The general case is then deduced by means of Bott's Reflection Lemma (Sect. 3.5). This last argument is quite standard; the technical tool used is the Serre-Hochschild spectral sequence (see App. A).

Section 2 presents the Hilbert space argument. We define in Sect. 2.1 a distinguished hermitian structure on the Lie algebra cohomology complex $\operatorname{Hom}_{\mathfrak{g}_{\mathbb{C}}}\left(\Lambda^{*}\left(\mathfrak{g}_{\mathbb{C}[z]} / \mathfrak{g}_{\mathbb{C}}\right) ; \mathscr{H} \otimes \vec{V}(\vec{z})\right)$ (cf. App. A), related to the Kähler metric on the flag variety $X$, determined by the hermitian curvature of $\mathscr{L}$. We then establish on this Hilbert space complex an identity of geometric origin, due to Nakano in the context of vector bundles over Kähler manifolds, where it relates the $(1,0)$ and $(0,1)$ Laplacians on $\Omega^{p, q}$. It reads, for us

$$
\bar{\partial} \bar{\partial}^{*}+\bar{\partial}^{*} \bar{\partial}=\square+(h+2 c) \cdot \operatorname{deg}-\sum_{a ; k} \theta_{k}\left(\xi_{a}\right) \otimes \operatorname{ad}_{\zeta_{a}(0)} \cdot H_{z_{k}}^{-1},
$$

where $\square$ is a non-negative operator, "deg" is the degree on $\Lambda^{*}(\ldots), \theta_{k}$ is the action of $\mathfrak{g}$ on $V_{k}$ and ad the action on $A^{*}(\ldots)$, while the $H_{z_{k}}$ are the infinitesimal generators of the $S L_{2}(\mathbb{R})$-rotations of the unit disk about the points $z_{k}$. When the $V_{k}$ 's are antidominant, we show that $\sum_{a} \theta_{k}\left(\xi_{a}\right) \otimes \operatorname{ad}_{\zeta_{a}(0)} \leqq(h+2 c-1)$. If, additionally, the points $z_{k}$ are inside the unit disk and near the boundary, $\sum_{k} H_{z_{k}}^{-1}<1+\varepsilon$ (cf. Appendix C). These two inequalities prove the positivity of the $\bar{\partial}$-Laplacian on higher degree forms (Theorem 2.2.3).

Section 3 handles the "honest" Lie algebra cohomology with coefficients in the formally completed representation $\hat{\mathscr{H}} \otimes \vec{V}(\vec{z})$. The connection with Sect. 2 is made 
in Sect. 3.3, where we prove that every cohomology class in the formally completed Lie algebra cohomology complex $\hat{\mathscr{C}}^{*}$ has a representative in the Hilbert space domain of the $\bar{\partial}$-operator. In other words, the obvious map from the cohomology of the Hilbert space complex to that of $\hat{\mathscr{C}}^{*}$ is surjective. Under the extra assumption, mentioned above, on the points $z_{k}$, case (c) of Theorem 0 now follows from the Hilbert space vanishing theorem.

The extra restriction on the position of the points $z_{k}$ makes little sense in the algebraic setting, and indeed we remove it in Sect. 3.4. The basic tool is the action of $\hat{\mathscr{H}}$ of the Virasoro algebra, specifically of the parabolic subalgebra of polynomial vector fields on $\mathbb{C}$, which allows us to move the points $z_{k}$ infinitesimally. To summarize the argument, with a fashionable degree of overkill: the spaces $H^{*}\left(\mathfrak{g}_{\mathbb{C}[z]}, \mathfrak{g} ; \hat{\mathscr{H}} \otimes \vec{V}(\vec{z})\right)$ admit a finite-dimensional presentation $(3.2 .7)$, be-

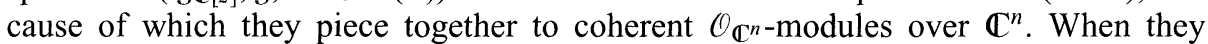
are restricted to the complement $Z(n)$ of all the diagonals, the Virasoro action can be used to give these $\mathscr{O}_{Z(n)}$-modules a $\mathscr{D}_{Z(n)}$-module structure. By a well-known theorem, they then form a vector bundle with a flat connection (the "KnizhnikZamolodchikov connection"). In particular, their dimension is constant (and finite), as long as the points $z_{k}$ are distinct.

Finally, the same finite-dimensional presentation shows that the Euler characteristic $\chi(\hat{\mathscr{H}} \otimes \vec{V}(\vec{z}))$ is well defined and stays constant, even when some of the points coalesce. This ends the proof of Theorem 0 .

\section{The Hilbert Space Vanishing Theorem}

We now prove that, in a Hilbert space version of the relative Lie algebra cohomology complex $\mathscr{C}^{*}\left(\mathfrak{g}_{\mathbb{C}[z]}, \mathfrak{g}_{\mathbb{C}} ; \mathscr{H} \otimes \vec{V}(\vec{z})\right)$, the $\bar{\partial}$-Laplacian is strictly positive on positive degree forms, if the representations $V_{k}$ are antidominant, and the points $z_{k}$ are near the boundary of the unit disk (Theorem 2.2.3).

2.0. Geometric Motivation: Nakano's Vanishing Theorem ${ }^{12}$. If $\mathbf{L}$ is a holomorphic line bundle over the compact complex mainfold $X$, then, under the assumption that $\mathbf{L}$ is positive, a well-known theorem of Kodaira guarantees the vanishing of higher cohomology of the twist $\mathbf{L} \otimes \mathbf{K}_{X}$ of $\mathbf{L}$ by the canonical bundle $\mathbf{K}_{X}$ of $X$. The proof is surprisingly simple. Let, in the Kähler metric determined by the curvature of L, $\square$ and $\bar{\square}$ denote the Laplacians of the $D$ - and $\bar{\partial}$-operators (coming from the hermitian connection $\nabla=D+\bar{\partial})$ on the $(p, q)$ complex $\Omega^{p, q}(X ; \mathbf{L})$ of $\mathbf{L}$-valued forms. They are related [S-S] by Nakano's identity $\bar{\square}=\square+(p+q)-\operatorname{dim}(X)$. In particular, restricting to the spaces $\Omega^{t o p, *}(X ; \mathbf{L})$ of top holomorphic degree forms, we have $\bar{\square}=\square+$ deg, with "deg" denoting the antiholomorphic degree ${ }^{13}$. There are thus no $\mathbf{L} \otimes \mathbf{K}_{X}$-valued harmonic $(0, q)$ forms in positive degree.

Kodaira's theorem was extended by Nakano to a class of hermitian holomorphic vector bundles $\mathbf{F}$ satisfying a (rather strong) positivity condition. The hermitian curvature $\Theta_{\mathbf{F}} \in \operatorname{End}(\mathbf{F}) \otimes \Lambda^{1,1}\left(\mathbf{T}_{X}\right)$ of $\mathbf{F}$ defines a hermitian form $T_{\mathbf{F}}$ on the bundle $\mathbf{F} \otimes \mathbf{T}_{X}$, by means of $T_{\mathbf{F}}(e \otimes \sigma, f \otimes \tau)=\left\langle e\left|\Theta_{\mathbf{F}}(\tau, \bar{\sigma})\right| f\right\rangle$.

\footnotetext{
12 A good reference for this material is the book by [S-S].

13 Caution: $\Omega^{t i p, *}(X ; \mathbf{L}) \cong \Omega^{0, *}\left(X ; \mathbf{L} \otimes \mathbf{K}_{X}\right)$ as $\bar{\partial}$-complexes and as hermitian spaces, but the $\square$-Laplacians on them, defined from the $D$-operators on the corresponding $(p, q)$-complexes, are not the same. We do require the operator $\square=D D^{*}$ on $\Omega^{(t), * *}(X ; \mathbf{L})$.
} 
Definition 2.0.1. $\mathbf{F}$ is called Nakano-positive if $T_{\mathbf{F}}$ is positive-definite.

This generalizes the usual notion of positivity for line bundles.

Using a Kähler metric on $X$ and the isomorphism $\mathbf{T}_{X} \cong\left(\overline{\mathbf{T}}_{X}\right)^{t}$ it produces, we may view $T_{\mathbf{F}}$ as a hermitian operator on $\mathbf{F} \otimes\left(\overline{\mathbf{T}}_{X}\right)^{t}$. It has a natural multilinear extension $T_{\mathbf{F}}^{\Lambda}$ to $\Omega^{t o p, *}(X ; \mathbf{F})$ by

$$
\begin{aligned}
T_{\mathbf{F}}^{\Lambda}\left(\kappa \cdot f \otimes \tau^{1} \wedge \cdots \wedge \tau^{q}\right)= & \kappa \cdot T_{\mathbf{F}}\left(f \otimes \tau^{1}\right) \wedge \cdots \wedge \tau^{q}+\cdots \\
& +\kappa \cdot \tau^{1} \wedge \cdots \wedge T_{\mathbf{F}}\left(f \otimes \tau^{q}\right),
\end{aligned}
$$

where $\kappa$ is a section of $\mathbf{K}_{X}, f$ one of $\mathbf{F}$, and the $\tau^{k}$ are $(0,1)$-forms. The $(1,0)$ and $(0,1)$ Laplacians $\square$ and $\bar{\square}$ of the hermitian connection, restricted to the complex $\Omega^{t o p, *}(X ; \mathbf{F})$, are related by Nakano's identity

$$
\bar{\square}=\square+T_{\mathbf{F}}^{\Lambda} \text {. }
$$

When $\mathbf{F}$ is Nakano-positive, it easily follows that $T_{\mathbf{F}}^{\Lambda}$ is a positive operator on positive-degree forms (cf. Remark 2.4.5). Because of Nakano's identity, there will be no harmonic forms, and all higher cohomology of $\mathbf{F} \otimes \mathbf{K}_{X}$ must vanish.

The relevance of these ideas lies in the fact that, as we shall prove below, on the flag variety $X=L G_{\mathbb{C}} / G_{\mathbb{C}}^{\Delta}$, the vector bundle $\mathbf{F}=\mathscr{L}^{\otimes(h+2 c)} \otimes \overrightarrow{\mathbf{V}}(\vec{z})$ is Nakanopositive when the $V_{k}$ 's are antidominant and the points $z_{k}$, inside the unit disk, are sufficiently far apart ${ }^{14}$. Further, the canonical bundle $\mathbf{K}_{X}$ turns out to be $\mathscr{L}^{\otimes(-2 c)}$, in a very concrete sense: Nakano's identity (2.4.7) on a representation of level $h$ involves the curvature of $\mathscr{L}^{\otimes(h+2 c)}$ (one should interpret this as $\mathscr{L}^{\otimes h}=\mathscr{L}^{\otimes(h+2 c)} \otimes$ $\left.\mathbf{K}_{X}\right)$. We "conclude" from here the vanishing of $H^{q}\left(X ; \mathscr{L}^{\otimes h} \otimes \overrightarrow{\mathbf{V}}(\vec{z})\right)$ for $q>0$. What we shall really deduce is the Hilbert space vanishing theorem 2.2 .3 , and this chapter is devoted to its proof.

2.1. The Setup. If $h$ is a positive integer, there is a natural Hilbert space structure on an irreducible negative energy representation $\mathscr{H}$ of $L G$ at level $(-h)$ [P-S, Ch. 11]. The space $\vec{V}(\vec{z}) \cong V_{1} \otimes \cdots \otimes V_{n}$ also has a hermitian structure, coming from the $G$-invariant inner products on the factors. We define an inner product on the Lie subalgebra $\mathfrak{g}_{+}:=\bigoplus_{n>0} \mathfrak{g}_{\mathbb{C}}(n)$ of positive Fourier modes in $L \mathfrak{g}_{\mathbb{C}}$ by the formula

$$
\left\langle\xi_{a}(m) \mid \xi_{b}(n)\right\rangle:=n \cdot \delta_{m, n} \cdot \delta_{a, b}
$$

Under the isomorphism $\mathfrak{g}_{+} \cong \mathfrak{g}_{\mathbb{C}[z]} / \mathfrak{g}_{\mathbb{C}}$, this corresponds to

$$
\langle\xi \mid \eta\rangle=\frac{1}{2 \pi \mathrm{i}} \int_{\Delta} \overline{\partial \xi} \wedge \partial \eta \quad \text { for } \xi, \eta \in \mathfrak{g}_{\mathbb{C}} .
$$

We note in particular the invariance of this inner product under the geometric action of $S L_{2}(\mathbb{R})$ on the space of holomorphic functions on the unit disk $\Delta$.

Remark. If $\mathfrak{g}_{+}$, suitably completed, is regarded as the antiholomorphic tangent space to the flag manifold $L G_{\mathbb{C}} / G_{\mathbb{C}}^{\Delta}$ at the identity, $(2.1 .1)$ is the Kähler metric determined by the hermitian curvature of $\mathscr{L}$.

\footnotetext{
14 In the hyperbnolic metric. One can thus accommodate any number of points; but the minimum hyperbolic distance between them increases with their number.
} 
Define now the Hilbert space cohomology complex, mentioned in Sect. 1.6, as

$$
\begin{aligned}
\mathscr{C}_{\mathrm{Hilb}}^{*}(\mathscr{H} \otimes \vec{V}(\vec{z})):= & \{\text { Hilbert-Schmidt operators in } \\
& \left.\operatorname{Hom}_{\mathfrak{g}_{\mathbb{C}}}\left(\Lambda^{*}\left(\mathfrak{g}_{\mathbb{C}[z]} / \mathfrak{g}_{\mathbb{C}}\right) ; \mathscr{H} \otimes \vec{V}(\vec{z})\right)\right\},
\end{aligned}
$$

the subspace of Hilbert-Schmidt operators in the standard complex of relative Lie algebra cohomology of the pair $\left(\mathfrak{g}_{\mathbb{C}[z]} / \mathfrak{g}_{\mathbb{C}}\right)$ with coefficients in $\hat{\mathscr{H}} \otimes \vec{V}(\vec{z})$. The spaces (2.1.2) are independent of $\vec{z}$, but there is an explicit $\vec{z}$-dependence in the coboundary operator:

$$
\bar{\partial}_{\bar{z}}=\sum_{m=1}^{\infty} \varepsilon^{a}(-m) \cdot\left(R\left(\xi_{a}(m)\right)+\frac{1}{2} \operatorname{ad}_{\xi_{a}(m)}^{t}\right)+\sum_{m=1}^{\infty} \varepsilon^{a}(-m) \cdot \theta_{\bar{z}}\left(\xi_{a}(m)\right) .
$$

Here, $\varepsilon^{a}(-m)$ is the operator of exterior multiplication by $\xi^{a}(-m) \in\left(\mathfrak{g}_{+}\right)^{t}, R\left(\xi_{a}(m)\right)$ stands for the action on the factor $\mathscr{H}, \mathrm{ad}_{*}^{t}$ denotes the co-adjoint action of $\mathfrak{g}_{+}$on $\left(\mathfrak{g}_{+}\right)^{t}$-or, rather, its multilinear extension to $\operatorname{Hom}_{\mathfrak{g}_{\mathbb{C}}}\left(\Lambda^{*}\left(\mathfrak{g}_{+}\right) ; \ldots\right)$; while in the second term,

$$
\theta_{\bar{z}}\left(\xi_{a}(m)\right):=\sum_{k} \theta_{k}\left(\xi_{a}\right) \cdot z_{k}^{m}
$$

is the action of the loop $\xi_{a}(m) \in \mathfrak{g}_{+}$on $V_{1}\left(z_{1}\right) \otimes \cdots \otimes V_{n}\left(z_{n}\right) ; \theta_{k}$ denotes the action of $\mathfrak{g}_{\mathbb{C}}$ on the factor $V_{k}$ alone. For $\theta$ to be a sensible operator, the points must be inside the unit disk.

2.2. Statement of the Vanishing Theorem. We now list the main properties of the operator $\bar{\partial}_{\bar{z}}$, including the vanishing theorem 2.2 .3 , the purpose of the present chapter. The remaining sections, and some of the appendices, are devoted to the proofs. We shall write $\bar{\partial}$ for $\bar{\partial}_{\bar{z}}$ in this section.

Proposition 2.2.1. If the points $z_{k}$ are inside the unit disk,

(i) $\bar{\partial}$ is a densely defined, closable operator on $\mathscr{C}_{\mathrm{Hilb}}^{*}$, with adjoint $\bar{\partial}^{*}$, and $\bar{\partial}^{2}=$ $\bar{\partial}^{*}=0$;

(ii) $\bar{\partial}+\bar{\partial}^{*}$ is a self-adjoint operator with domain $\operatorname{Dom}(\bar{\partial}) \cap \operatorname{Dom}\left(\bar{\partial}^{*}\right)$;

(iii) $\operatorname{Ker}(\bar{\partial}) / \operatorname{Ran}(\bar{\partial})^{c} \cong \operatorname{Ker}\left(\bar{\partial}+\bar{\partial}^{*}\right)$, where $\operatorname{Ran}(\bar{\partial})^{c}$ is the closure of the range of $\bar{\partial}$.

(In other words, the cohomology is represented by harmonic forms.)

Proof. Parts (i) and (ii) will be covered in Sect. 2.3. Part (iii) is a general consequence of our context: $\operatorname{Ran}(\bar{\partial})^{c} \subset \operatorname{Ker}(\bar{\partial}), \operatorname{Ker}\left(\bar{\partial}+\bar{\partial}^{*}\right)=\operatorname{Ker}(\bar{\partial}) \cap \operatorname{Ker}\left(\bar{\partial}^{*}\right)$, and $\operatorname{Ker}\left(\bar{\partial}^{*}\right)=\left(\operatorname{Ran}(\bar{\partial})^{c}\right)^{\perp}$.

The operator $\bar{\partial}$ is quite well-behaved, but this is more difficult to prove:

Proposition 2.2.2. The range of $\bar{\partial}$ is closed. Equivalently, the essential spectrum of $\bar{\partial}+\bar{\partial}^{*}$ is bounded away from zero. (We are, again, assuming that the points $z_{k}$ are inside the unit disk.)

Proposition (2.2.2) implies that the cohomology of $\bar{\partial}$ is finite-dimensional, and that the Euler characteristic doesn't depend on the points. We shall not use it in this paper, and thus do not prove it here; the argument in the next section will go by a different route. Finally, we state the main theorem of this section: 
Vanishing Theorem (2.2.3). Assume that

(a) The representations $V_{k}$ are antidominant at level $h$;

(b) The points $z_{k}$ are inside the unit disk, and sufficiently far apart in the hyperbolic metric (e.g., near the boundary, but not near each other);

Then, there is no higher cohomology: $\operatorname{Ran}(\bar{\partial})$ is dense in $\operatorname{Ker}(\bar{\partial})$ in $\mathscr{C}_{\mathrm{Hilb}}^{q}$, for $q>0$.

Recall that (a) means $\lambda_{k} \cdot \alpha_{\max } \leqq h+1$ for the highest weights $\lambda_{k}$ of the $V_{k}$ 's.

The proof of the vanishing theorem is contained in Sect. 2.4 and Sect. 2.5. From (2.2.2) it follows that, in fact, $\operatorname{Ran}(\bar{\partial})=\operatorname{Ker}(\bar{\partial})$; but we shall not require this result.

2.3. Elementary Hilbert Space Properties. Using the energy gradings on $\mathscr{H}$ and $\mathfrak{g}_{+}$, we can split $\mathscr{C}_{\text {H1lb }}^{*}$ as an orthogonal sum of finite-dimensional negative energy eigenspaces. By (2.1.3) and (2.1.4), we can write

$$
\bar{\partial}_{\bar{z}}=\bar{\partial}_{0}+\sum_{\substack{1 \leqq k \leqq n \\ m>0}} z_{k}^{m} \cdot \bar{\partial}_{k,-m}
$$

where $\bar{\partial}_{k,-m}:=\sum \varepsilon^{a}(-m) \cdot \theta_{k}\left(\xi_{a}\right)$. The operator $\bar{\partial}_{k,-m}$ decreases the energy by $(-m)$, while $\bar{\partial}_{0}$ is simultaneously the energy-preserving component of $\bar{\partial}_{\bar{z}}$, and the $\bar{\partial}$-operator for $\vec{z}=0$. Clearly,

$$
\left|z_{k}\right|^{m} \cdot\left\|\bar{\partial}_{k,-m}\right\|=O\left(\mu^{m}\right) \text {, if } \mu=\max \left\{\left|z_{k}\right|\right\} \text {. }
$$

In particular, it follows that the difference $\delta_{\bar{z}}=\bar{\partial}_{\bar{z}}-\bar{\partial}_{0}$ is a bounded operator as long as the points $z_{k}$ are all inside the unit disk.

Also let $\bar{\partial}_{0}^{*}$ be the formal hermitian adjoint

$$
\bar{\partial}_{0}^{*}:=\sum_{m=1}^{\infty}\left(R\left(\xi_{a}(m)\right)^{*}+\frac{1}{2}\left(\operatorname{ad}_{\xi_{a}(m)}^{t}\right)^{*}\right) \cdot \varepsilon^{a}(-m)^{*},
$$

and $\bar{\partial}_{\vec{z}}^{*}:=\bar{\partial}_{0}^{*}+\delta_{\vec{z}}^{*}$. Note that only a finite number of terms survive when $\bar{\partial}_{0}^{*}$ acts on a finite-energy form.

The operators $\bar{\partial}_{0}$ and $\bar{\partial}_{0}^{*}$ are block-diagonalized by the energy decomposition of $\mathscr{C}_{\mathrm{H} \text { lb }}^{*}$, and as such they are quite well behaved.

\section{Proposition 2.3.4.}

(i) $\bar{\partial}_{0}$ extends naturally to a closed operator with domain

$$
\mathscr{D}:=\left\{\phi=\sum \phi(-m) \in \mathscr{C}_{\text {Hilb }}^{*} \mid \sum\left\|\bar{\partial}_{0} \phi(-m)\right\|^{2}<\infty\right\} .
$$

(ii) Its hermitian adjoint $\bar{\partial}_{0}^{*}$, a closed operator as well, has the domain

$$
\mathscr{D}^{*}:=\left\{\phi=\sum \phi(-m) \in \mathscr{C}_{\mathrm{Hilb}}^{*} \mid \sum\left\|\bar{\partial}_{0}^{*} \phi(-m)\right\|^{2}<\infty\right\} \text {. }
$$

(iii) $\bar{\partial}_{0}+\bar{\partial}_{0}^{*}$ is a self-adjoint operator on the domain $\mathscr{D} \cap \mathscr{D}^{*}$, and is the closure of the operator $\bar{\partial}_{0}+\bar{\partial}_{0}^{*}$, defined a priori on finite-energy forms.

(iv) Let $\bar{\square}_{0}:=\left(\bar{\partial}_{0}+\bar{\partial}_{0}^{*}\right)^{2}$. Then $\bar{\partial}_{0}^{2}=\left(\bar{\partial}_{0}^{*}\right)^{2}=0$ and $\bar{\square}_{0}=\bar{\partial}_{0} \bar{\partial}_{0}^{*}+\bar{\partial}_{0}^{*} \bar{\partial}_{0}$. 
Proof. All the statements are obvious except (iii). The domain of the closure of $\bar{\partial}_{0}+\bar{\partial}_{0}^{*}$ is

$$
\left\{\phi=\sum \phi(-m) \in \mathscr{C}_{\mathrm{H} 1 \mathrm{~b}}^{*} \mid \sum\left\|\left(\bar{\partial}_{0}+\bar{\partial}_{0}^{*}\right) \phi(-m)\right\|^{2}<\infty\right\}
$$

But $\left\|\left(\bar{\partial}_{0}+\bar{\partial}_{0}^{*}\right) \phi(-m)\right\|^{2}=\left\|\bar{\partial}_{0} \phi(-m)\right\|^{2}+\left\|\bar{\partial}_{0}^{*} \phi(-m)\right\|^{2}$, by (iv), and so (2.3.5) is precisely $\mathscr{D} \cap \mathscr{D}^{*}$.

Corollary 2.3.6. If all the points $z_{k}$ are inside the unit disk, then

(i) $\bar{\partial}_{\vec{z}}$ extends naturally to a closed operator with domain $\mathscr{D}$.

(ii) Its hermitian adjoint $\bar{\partial}_{\vec{z}}^{*}$, a closed operator as well, has the domain $\mathscr{D}^{*}$.

(iii) $\bar{\partial}_{\vec{z}}+\bar{\partial}_{\vec{z}}^{*}$ is a self-adjoint operator on the domain $\mathscr{D} \cap \mathscr{D}^{*}$, and is the closure of $\bar{\partial}_{\vec{z}}+\bar{\partial}_{\vec{z}}^{*}$, which is defined a priori only on finite-energy forms.

(iv) $\bar{\partial}_{\vec{z}}^{2}=\left(\bar{\partial}_{\vec{z}}^{*}\right)^{2}=0$ and $\bar{\square}_{\vec{z}}:=\left(\bar{\partial}_{\vec{z}}+\bar{\partial}_{\vec{z}}^{*}\right)^{2}=\bar{\partial}_{\vec{z}} \bar{\partial}_{\vec{z}}^{*}+\bar{\partial}_{\vec{z}}^{*} \bar{\partial}_{\vec{z}}$.

Proof. The differences $\delta_{\vec{z}}=\bar{\partial}_{\vec{z}}-\bar{\partial}_{0}$ and $\delta_{\vec{z}}^{*}=\bar{\partial}_{\vec{z}}^{*}-\bar{\partial}_{0}^{*}$ are bounded, and thus (i), (ii) and (iii) follow from the corresponding statements in Prop. 2.3.4. To give an honest proof of (iv) from the formula $\bar{\partial}_{\vec{z}}^{2}=0$, which holds in the formally completed complex $\hat{\mathscr{C}}^{*}$, one must show that the image $\delta_{\vec{z}}(\phi)$ of a finite energy form $\phi$ lies in the domains $\mathscr{D}$ and $\mathscr{D}^{*}$ of $\bar{\partial}_{0}$ and $\bar{\partial}_{0}^{*}$ (so that the compositions $\bar{\partial}_{0} \circ \delta_{\vec{z}}, \bar{\partial}_{0}^{*} \circ \delta_{\bar{z}}$, etc. make sense). We leave it to the patient reader to verify that the norm of $\bar{\partial}_{0}$ on energy eigenforms is polynomially bounded in the energy. To complete the argument, one then observes that by (2.3.2), the pure energy modes of $\delta_{\vec{z}}(\phi)$ have exponentially decaying norms.

2.4. Nakano's Identity. We now give a Lie algebra version of Nakano's identity. The Laplacian $\bar{\square}_{\vec{z}}$ will be expressed in terms of the degree, of the operator $T_{\vec{V}(\vec{z})}^{A}$ constructed from the curvature of the holomorphic vector bundle $L G_{\mathbb{C}} \times_{G_{\mathbb{C}}^{A}} \vec{V}(\vec{z})$ (2.4.1-2.4.4), and of a non-negative operator $\square$ (2.4.6).

Let $\theta_{E}: \mathfrak{g}_{\mathbb{C}[z]} \rightarrow \operatorname{End}(E)$ be a finite-dimensional holomorphic representation of $\mathfrak{g}_{\mathbb{C}[z]}$, restricting to a skew-adjoint representation of $\mathfrak{g}$. (We shall shortly set $E=$ $\vec{V}(\vec{z})$, thus $\theta_{E}=\theta_{\vec{z}}$ as in 2.1 .4 , although the formula we prove holds for any $E$, at least in a formal sense.) Using the standard real structure on $L \mathfrak{g}_{\mathbb{C}}$ (in which the loops mapping the unit circle into the skew-adjoint algebra $g$ are purely imaginary), we can extend the map $\theta_{E}$ by hermitian conjugation to a linear map $\theta_{E}: \mathfrak{g}_{\mathbb{C}\left[z, z^{-1}\right]} \rightarrow$ $\operatorname{End}(E)$; in other words, we set $\theta_{E}\left(\xi \cdot z^{-p}\right):=\theta_{E}\left(\xi^{*} \cdot z^{p}\right)^{*}$, for $p \geqq 0$ and $\xi \in \mathfrak{g}_{\mathbb{C}}$. The failure of the extended map $\theta_{E}$ to be a representation can be measured by a hermitian symmetric form on $E \otimes \overline{\mathfrak{g}_{+}}$,

$$
T_{E}(e \otimes \sigma, f \otimes \tau):=\left\langle e\left|\left[\theta_{E}(\tau), \theta_{E}(\bar{\sigma})\right]-\theta_{E}([\tau, \bar{\sigma}])\right| f\right\rangle
$$

for $e, f \in E$ and $\sigma, \tau \in \overline{\mathfrak{g}_{+}}$.

Remark. If we identify $\overline{\mathfrak{g}_{+}}=\bigoplus_{m<0} \mathfrak{g}_{\mathbb{C}}(m)$ and $\mathfrak{g}_{+}=\bigoplus_{m>0} \mathfrak{g}_{\mathbb{C}}(m)$ with the $(1,0)$ and $(0,1)$ parts of the complexified tangent space to $X=L G_{\mathbb{C}} / G_{\mathbb{C}}^{\Delta}$ at the identity, then the $(1,1)$-form $\Theta_{E} \in \operatorname{Hom}\left(\overline{\mathfrak{g}_{+}} \otimes \mathfrak{g}_{+} ; \operatorname{End}(E)\right) \cong \Lambda^{1,1}\left(T_{1} X\right) \otimes \operatorname{End}(E)$, given by 


$$
\Theta_{E}:=\sum_{p, q>0}\left\{\left[\theta_{E}\left(\xi_{a}(-p)\right), \theta_{E}\left(\check{\zeta}_{b}(q)\right)\right]-\theta_{E}\left(\left[\xi_{a}, \xi_{b}\right](q-p)\right)\right\} \otimes \xi^{a}(p) \wedge \xi^{b}(-q)
$$

is the curvature of the hermitian holomorphic vector bundle $L G_{\mathbb{C}} \times_{G_{\mathbb{C}}^{A}} E$ over $X$. The relation between $\Theta_{E}$ and $T_{E}$ is given, as in Sect. 2.0, by

$$
T_{E}(e \otimes \sigma, f \otimes \tau)=\left\langle e\left|\Theta_{E}(\tau, \bar{\sigma})\right| f\right\rangle,
$$

for $e, f \in E$ and $\sigma, \tau \in \overline{\mathfrak{g}_{+}}$.

Assume that the form $T_{E}$ is bounded in the Kähler metric (2.1.1) on $\mathfrak{g}_{+}$. This is certainly the case for $E=\vec{V}(\vec{z})$, because of the exponential decay (2.1.4) of the $\theta_{\vec{z}}\left(\check{\zeta}_{a}(m)\right)$ 's. This form corresponds then to a bounded hermitian operator on $E \otimes \overline{\mathfrak{g}_{+}}$. Using the Hilbert space isomorphism $\overline{\mathfrak{g}_{+}} \cong\left(\mathfrak{g}_{+}\right)^{t}$, we shall view it instead as an operator on $\operatorname{Hom}\left(\mathfrak{g}_{+} ; E\right)$, extending to a multilinear operator $T_{E}^{A}$ on $\operatorname{Hom}\left(\Lambda^{*}\left(\mathfrak{g}_{+}\right) ; E\right)$ :

$$
T_{E}^{A}\left(e \otimes \gamma^{1} \wedge \gamma^{2} \wedge \cdots \wedge \gamma^{\prime}\right)=\sum_{k} \gamma^{1} \wedge \cdots \wedge \gamma^{h-1} \wedge T_{E}^{A}\left(e \otimes \gamma^{k}\right) \wedge \gamma^{k+1} \wedge \cdots \wedge \gamma^{\prime \prime}
$$

for $e \in E$ and $\gamma^{\prime}, \ldots, \gamma^{\prime \prime} \in\left(\mathfrak{g}_{+}\right)^{t}$.

Remark 2.4.5. We have $T_{E}^{\Lambda} \geqq 0$ if $T_{E} \geqq 0$. To see this, let $T^{*}\left(\mathfrak{g}_{+}\right)$be the tensor algebra of $\mathfrak{G}_{+}$, and notice that the same formula (2.4.4) can be used to define an extension $T_{E}^{\otimes}$ of $T_{E}$ to $\operatorname{Hom}\left(T^{*}\left(\mathfrak{g}_{+}\right) ; E\right)$. Then, $T_{E}^{\otimes}$ is a sum of non-negative operators, and restricts to $T_{E}^{A}$ on $\operatorname{Hom}\left(\Lambda^{*}\left(\mathfrak{g}_{+}\right) ; E\right)$. Similarly, if $T_{E} \geqq C$ for a constant $C$, then $T_{E}^{A} \geqq C \cdot p$ on $\operatorname{Hom}\left(\Lambda^{p}\left(\mathfrak{g}_{+}\right) ; E\right)$.

When $E=\vec{V}(\vec{z})$, we shall give an explicit formula and an estimate for $T_{E}$ in Sect. 2.5.

Finally, we define the operator $\square: \mathscr{G}_{\mathrm{Hilb}}^{*}(\mathscr{H} \otimes \vec{V}(\vec{z})) \rightarrow \mathscr{G}_{\mathrm{H} \text { lb }}^{*}(\mathscr{H} \otimes \vec{V}(\vec{z}))$ by

$$
\begin{aligned}
& \left.\square:=\sum_{m=1}^{\infty}\left(R\left(\xi_{a}(-m)\right)+0\right)_{\Xi}\left(\check{\zeta}_{a}(-m)\right)+\operatorname{ad}_{\xi_{a}(-m)}^{t}\right) \\
& \left.\times\left(R\left(\check{\zeta}_{a}(-m)\right)+0\right)_{\Xi}\left(\check{\zeta}_{a}(-m)\right)+\operatorname{ad}_{\check{\zeta}_{a}(-m)}^{t}\right)^{*} .
\end{aligned}
$$

As in (2.1.3), we have written $R($.$) for the action of \mathfrak{g}_{\mathbb{C}\left[z, z^{-1}\right]}$ on $\mathscr{H}$. Also, $\theta_{\vec{z}}\left(\xi_{a}(-m)\right)=-\theta_{\vec{z}}\left(\xi_{a}(m)\right)^{*}$, as defined earlier, while $\operatorname{ad}_{\xi_{a}(-m)}^{t}$ stands for the coadjoint action of $\xi_{a}(-m)$, truncated to $\mathfrak{g}_{+}$:

$$
\operatorname{ad}_{\breve{c},(-m)}^{t}(\eta(-k)):=\left(\operatorname{ad}_{\breve{s} a}^{\prime}(\eta)\right)(-m-k)
$$

for $\eta(-k) \in\left(\mathfrak{g}_{+}\right)^{t}$ (which means that $\eta \in\left(\mathfrak{g}_{\mathbb{C}}\right)^{t}$ and $\left.k>0\right)$. One should note that $\operatorname{ad}_{-a(-m)}^{\prime} \neq-\left(\operatorname{ad}_{\dot{c}_{a}(m)}^{t}\right)^{*}$.

At least formally, $\square$ is a non-negative self-adjoint operator. One can check directly that the finite-energy forms in $\mathscr{C}_{\mathrm{H} I \mathrm{lb}}^{*}$ are in its Hilbert space domain ${ }^{15}$.

15 Notice that, aside from terms involving $\theta_{\Xi^{\prime}}($.$) , only finitely many terms survive when the sum$ (1.3.5) is applied to a finite-energy form. One then recalls the exponential decay 1.2.1 of the energy modes of $\theta_{\Sigma}$. The operators $\mathrm{ad}_{\xi_{a}(-m)}^{t}$ are actually bounded on forms of given degree, while the norm of $R\left(\xi_{a}(-m)\right)$, on forms of fixed energy, increases as $\sqrt{m}$. This last statement follows from the identity $R\left(\xi_{a}(-m)\right)^{*} \cdot R\left(\xi_{a}(-m)\right)=h \cdot m+R\left(\xi_{a}(-m)\right) \cdot R\left(\xi_{a}(-m)\right)^{*}$, where the last term is bounded on forms of a given energy. The details of the calculation are best left to the skeptical but patient reader. 
Fortunately, we do not need to construct by hand a non-negative self-adjoint extension of $\square$; the following identity expresses $\square$ in terms of $\square$ and $T_{\vec{V}(\vec{z})}^{A}$.

Proposition 2.4.7. (“Nakano's Identity”). On $\mathscr{C}_{\mathrm{H} l \mathrm{lb}}^{*}(\mathscr{H} \otimes \vec{C}(\vec{z}))$, we have

$$
\bar{\square}=\square+(h+2 c) \cdot \operatorname{deg}+T_{\vec{V}(\vec{z})}^{\Lambda},
$$

where "deg" is the degree operator on forms, and $T_{\vec{V}(\vec{z})}^{\Lambda}$, given by (2.4.4), acts trivially on the factor $\mathscr{H}$.

Proof. Appendix B.1.

2.5. Nakano Positivity. We are ready to prove the vanishing theorem (2.2.3).

Proposition 2.5.1. Let $\beta<1$, and assume the representations $V_{k}$ are antidominant at level $h$. Then, if the points $z_{k} \in \Delta$ are sufficiently far apart in the hyperbolic metric on $\Delta$,

$$
T_{\vec{V}(\vec{z})}+(h+2 c)>\beta .
$$

Proof of the Vanishing Theorem 2.2.3. The previous proposition, coupled with Remark (2.4.5), implies that $T_{\vec{V}(\vec{z})}^{A}+(h+2 c) \cdot \operatorname{deg}>\beta \cdot \operatorname{deg}>0$ on positivedegree forms. Nakano's identity shows that $\bar{\square}>0$.

Proof of Proposition 2.5.1. We give an explicit formula for $T_{\vec{V}(\vec{z})}$. For starters, notice that

$$
T_{\vec{V}(\vec{z})}=T_{V_{1}\left(z_{1}\right)}+\cdots+T_{V_{n}\left(z_{n}\right)}
$$

on $\operatorname{Hom}\left(\mathfrak{g}_{+} ; \vec{V}(\vec{z})\right)=V_{1}\left(z_{1}\right) \otimes \cdots \otimes V_{n}\left(z_{n}\right) \otimes\left(\mathfrak{g}_{+}\right)^{t}$, because in (2.4.1), we have $\theta_{\vec{z}}=\theta_{1}+\cdots+\theta_{n^{\prime}}$ and $\theta_{i}$ commutes with $\theta_{j}$ for $i \neq j$. (In other words the curvature $\Theta_{\vec{V}(\vec{z})}$ of the bundle $\vec{V}(\vec{z})$ over $L G_{\mathbb{C}} / G_{\mathbb{C}}^{4}$ is the sum of the curvatures $\Theta_{V_{k}\left(z_{k}\right)}$ of the factors $V_{k}\left(z_{k}\right)$; cf. 2.4.3.)

The operator $T_{V_{k}\left(z_{k}\right)}$ has a very simple form when $z_{k}=0$. Let $H_{0}$ be the selfadjoint infinitesimal generator of the rotation action on $\left(\mathfrak{g}_{+}\right)^{t}$ :

$$
H_{0}\left(\xi^{a}(-m)\right):=m \cdot \xi^{a}(-m) .
$$

The resolvent $H_{0}^{-1}$ is a compact positive operator, with spectrum $\{1 / m\}_{m>0}$.

Lemma 2.5.3.

$$
T_{V_{k}(0)}=-\sum_{a} \theta_{k}\left(\xi_{a}\right) \otimes \operatorname{ad}_{\xi_{a}(0)}^{t} \cdot H_{0}^{-1}
$$

Proof. In short, the operator $H_{0}^{-1}$ compensates for the presence of the factor " $m$ " in the energy $m$ directions in the metric (2.1.1) on $X$. The formal proof is by direct verification. Using the Kähler metric, we identify $\left(\mathfrak{g}_{+}\right)^{t}$ with $\overline{\mathfrak{g}_{+}}$, and take $e \otimes \xi(-p)$ and $f \otimes \eta(-q)$ in $V_{k}(0) \otimes \overline{\mathfrak{g}_{+}}$. Then,

$$
\begin{aligned}
T_{V_{k}(0)}(e \otimes \xi(-p), f \otimes \eta(-q))= & \langle e|\left[\theta_{k}(\eta(-q)), \theta_{k}\left(\xi^{*}(p)\right)\right] \\
& -\theta_{k}\left(\left[\eta, \xi^{*}\right](p-q)\right)|f\rangle \\
= & -\delta_{p, q} \cdot\left\langle e\left|\theta_{k}\left(\left[\eta, \xi^{*}\right]\right)\right| f\right\rangle,
\end{aligned}
$$


since, at $z_{k}=0, \theta_{k}(\xi(m))=0$ for $m \neq 0$. But we have (with summation over $a$ implied)

$$
\begin{aligned}
\left\langle e\left|\theta_{k}\left(\left[\eta, \xi^{*}\right]\right)\right| f\right\rangle & =-\left\langle e \mid \theta_{k}\left(\xi_{a}\right)(f)\right\rangle\left\langle\xi_{a} \mid\left[\eta, \xi^{*}\right]\right\rangle \\
& =-\left\langle e \mid \theta_{k}\left(\xi_{a}\right)(f)\right\rangle\left\langle\left[\xi_{a}, \xi\right] \mid \eta\right\rangle=\left\langle e \mid \theta_{k}\left(\xi_{a}\right)(f)\right\rangle\left\langle\xi \mid\left[\xi_{a}, \eta\right]\right\rangle
\end{aligned}
$$

Thus,

$$
\begin{aligned}
T_{V_{k}(0)}(e \otimes \xi(-p), f \otimes \eta(-q))= & -\delta_{p, q} \cdot\left\langle e \mid \theta_{k}\left(\xi_{a}\right)(f)\right\rangle\left\langle\xi \mid\left[\xi_{a}, \eta\right]\right\rangle \\
= & -\frac{1}{q} \cdot\left\langle e \mid \theta_{k}\left(\xi_{a}\right)(f)\right\rangle\left\langle\xi(-p) \mid\left[\xi_{a}, \eta\right](-q)\right\rangle \\
= & -\langle e \otimes \xi(-p)| \sum_{a} \theta_{k}\left(\xi_{a}\right) \\
& \otimes \operatorname{ad}_{\xi_{a}(0)} \cdot H_{0}^{-1}|f \otimes \eta(-q)\rangle .
\end{aligned}
$$

Let now $z_{k} \in \Delta$ be arbitrary, and pick a Möbius transformation $\phi \in S L_{2}(\mathbb{R})$ taking 0 to $z_{k}$. In constructing the operator $T_{E}$ in Sect. 2.4, we have only used

- the Lie algebra structure of $L \mathfrak{g}_{\mathbb{C}}$,

- the real structure on $L \mathfrak{g}_{\mathbb{C}}$,

- the Hilbert space metric (2.1.1) on $\mathfrak{g}_{+} \cong \mathfrak{g}_{\mathbb{C}}^{4} / \mathfrak{g}_{\mathbb{C}}$.

These are all preserved by the geometric action of $\phi$. Because $\phi^{*}\left(V_{k}(0)\right)=V_{k}\left(z_{k}\right)$ as $\mathfrak{g}_{\mathbb{C}}^{A}$-representations, it follows that we must have $\phi \circ T_{V_{k}(0)} \circ \phi^{-1}=T_{V_{k}\left(z_{k}\right)}$. Denoting by $H_{z_{k}}$ the infinitestimal $S L_{2}(\mathbb{R})$-rotation $\phi \circ H_{0} \circ \phi^{-1}$ about $z_{k}$, we have proved the following proposition.

Proposition 2.5.4. $T_{\vec{V}(\vec{z})}=-\sum_{k ; a} \theta_{k}\left(\xi_{a}\right) \otimes \operatorname{ad}_{\xi_{a}(0)}^{t} \cdot H_{z_{k}}^{-1}$ on $V_{t}\left(z_{1}\right) \otimes \cdots \otimes V_{n}\left(z_{n}\right) \otimes$ $\left(\mathfrak{g}_{+}\right)^{t}$.

\section{Proposition 2.5.5.}

(i) $\sum_{a} \theta_{k}\left(\xi_{a}\right) \otimes \operatorname{ad}_{\xi_{a}}^{t} \leqq\left(\lambda_{k}+2 \rho\right) \cdot \alpha_{\max }$ on $V_{k} \otimes\left(\mathfrak{g}_{\mathbb{C}}\right)^{t}$;

(ii) For any $\varepsilon>0$, we have $\sum_{k} H_{z_{k}}^{-1}<1+\varepsilon$ if the points $z_{k} \in \Delta$ are sufficiently far apart in the hyperbolic metric.

Proof. Appendix C.

End of proof of Proposition 2.5.1. Choose the points $z_{k}$ such that part (ii) in Prop. 2.5.5 holds with $\varepsilon=(1-\beta) /(h+2 c-1)$. Recall also that $c=\rho \cdot \alpha_{\max }+1$. Then,

$$
\begin{aligned}
T_{\vec{V}(\vec{z})} & =-\sum_{k ; a} \theta_{k}\left(\xi_{a}\right) \otimes \operatorname{ad}_{\xi_{a}(0)}^{t} \cdot H_{z_{k}}^{-1} \geqq-\sum_{k}\left(\lambda_{k}+2 \rho\right) \cdot \alpha_{\max } \cdot H_{z_{k}}^{-1} \\
& =-\sum_{k}\left(\lambda_{k} \cdot \alpha_{\max }+2 c-2\right) \cdot H_{z_{k}}^{-1} \geqq-\sum_{k}(h+2 c-1) \cdot H_{z_{k}}^{-1} \\
& >-(h+2 c-1) \cdot(1+\varepsilon)=-(h+2 c-1)-(1-\beta) \\
& =\beta-(h+2 c) .
\end{aligned}
$$




\section{Lie Algebra Cohomology}

In this chapter, we use the Hilbert space vanishing theorem (2.2.3) to determine, for a simple Lie algebra $\mathfrak{g}$, the relative Lie algebra cohomology

$$
H^{*}\left(\mathfrak{g}_{\mathbb{C}[z]}, \mathfrak{g}_{\mathbb{C}} ; \hat{\mathscr{H}} \otimes V_{1}\left(z_{1}\right) \otimes \cdots \otimes V_{n}\left(z_{n}\right)\right) .
$$

As in Sect. 1.4, $\hat{\mathscr{H}}$ is a formally completed irreducible negative energy representation of $L \mathfrak{g}$ at level $(-h)$ and the $V_{k}$ 's are irreducible representations of $\mathfrak{g}$, on which $\mathfrak{g}_{\mathbb{C}[z]}$, the Lie algebra of $\mathfrak{g}$-valued polynomials, acts via evaluation at the points $z_{k}$.

In Sect. 3.1 we collect some useful properties of the representation $\hat{\mathscr{H}}$; they are summarized in Prop. 3.1.5. Section 3.2 describes an injective resolution of $\hat{\mathscr{H}}$ and $\hat{\mathscr{H}} \otimes \vec{V}(\vec{z})$ in the category $\mathscr{C}\left(\mathfrak{g}_{\mathbb{C}[z]}, G\right)$ (Sect. 1.4). We use it to reduce the calculation of the cohomology (3.0.1) to a finite-dimensional problem; this plays an important, if somewhat technical role in Sect. 3.4, where, in order to conclude that the dimensions $h^{k}$ are independent of the choice of distinct points $z_{k} \in \mathbb{C}$, we exponentiate the Virasoro action on $H^{*}$. The finite-dimensional reduction also shows, without using the Fredholm properties of $\bar{\partial}$, that the Euler characteristic $\chi(\mathscr{H} \otimes \vec{V}(\vec{z}))$ does not depend on the points $z_{k}$, whether they are distinct or not. Section 3.3 contains the most important lemma of this chapter (Prop. 3.3.1), relating the Hilbert space theorem of Chapter 2 to the vanishing of the algebraic cohomology discussed here. Section 3.5 describes the cohomology (3.0.1) in the general case, with no restrictions on the representations $V_{k}$. This is the "Bott" part of the BorelWeil-Bott theorem, and the argument is a Lie algebra translation of Bott's original proof. Finally, in Sect. 3.6, as an application of this theorem, we determine of the fusion rules on $\mathbb{P}^{1}$.

3.1. The Formally Completed Representation $\hat{\mathscr{H}}$. Recall that $G$ is a compact simply connected Lie group with Lie algebra $\mathfrak{g}$, while $G_{\mathbb{C}}$ and $\mathfrak{g}_{\mathbb{C}}$ denote their complexifications. For a $\mathbb{C}$-algebra $A$, we shall write $G_{A}$ or $G(A)$, and $\mathfrak{g}_{A}$ of $\mathfrak{g}(A)$ for the corresponding objects over $A$. We are especially interested in the cases $A=\mathscr{M}_{x}$, the germs of meromorphic functions at $x$ on $\mathbb{P}^{1}, A=\mathcal{O}_{x}$, the subring of regular functions, or, later, $A=\mathbb{C}\left[z,\left(z-z_{1}\right)^{-1}, \ldots,\left(z-z_{n}\right)^{-1}\right]$, the regular functions on a punctured $\mathbb{P}^{1}$. We shall, however, also keep the old notations $L G_{\mathbb{C}}$ and $G_{\mathbb{C}}^{4}$ of Sect. 1 .

Take a disk $\Delta$ around a point $x$ in a Riemann surface ${ }^{16}$. It corresponds to the unit disk in $\mathbb{C}$, for some local coordinate $z$ on the surface, centered at $x$. Given an antidominant regular representation $E$ of $G$, the space of sections of the holomorphic vector bundle

$$
\mathbf{E}(x)=\left(L G_{\mathbb{C}} \times_{G_{\mathbb{C}}} E(x)\right)
$$

over the flag variety $X^{\Delta}=L G_{\mathbb{C}} / G_{\mathbb{C}}^{\Delta}$ forms an irreducible PER of $L G$; but the topology on this space depends in a non-trivial manner on the choice of $\Delta$.

Consider instead the formal completion $\hat{\mathscr{H}}_{E}$, or $\hat{\mathscr{H}}_{E}(x)$, of Sect. 1.4 - the direct product of the (finite-dimensional) energy eigenspaces in $\mathscr{H}_{E}$. It is clearly acted upon by the Lie algebra $\mathfrak{g}\left(\mathscr{M}_{x}\right)$, since the finite principal part of a meromorphic loop will give a bounded downward energy shift. Unlike $\mathscr{H}_{E}$, the completion

16 Which in the end will be $\mathbb{P}^{1}$, but we do not need this restriction for the moment. 
$\hat{\mathscr{H}}_{E}(x)$ depends only on an infinitesimal neighbourhood of the point $x$, and not on the choice of the local coordinate. This follows from the fact the group of germs of holomorphic coordinate changes about $x$ acts on $\hat{\mathscr{H}}_{E}(x)$. Indeed, the Virasoro generators $\left\{L_{k} \mid k \geqq 0\right\}$, corresponding to holomorphic vector fields vanishing at $x$, do not decrease the energy, and so their action on $\hat{\mathscr{H}}_{E}(x)$ can be exponentiated. In coordinate-invariant terms, $\hat{\mathscr{H}}_{E}(x)$ is topologised by the energy co-filtration, formed by the spans of vectors of energy above given cutoffs ${ }^{17}$.

Remark 3.1.1. There is another way to understand this coordinate-independence, and the following alternative description of $\hat{\mathscr{H}}_{E}$ will be helpful in Sect. 3.6. Let $X^{a l y}$ denote the algebraic flag manifold $G\left(\mathscr{M}_{x}\right) / G\left(\mathcal{O}_{x}\right)$. It is naturally contained in $X^{\Delta}$ as the union of the Schubert cells ${ }^{18}[\mathrm{P}-\mathrm{S}]$. There is the natural co-filtration on $\mathscr{H}_{E}$ by the kernels

$$
\mathscr{H}_{E}^{\mathscr{C}}=\left\{\sigma \in \mathscr{H}_{E} \mid \sigma \text { vansihes on } \mathscr{C}\right\}
$$

of the restriction maps to the Schubert cells $\{\mathscr{C}\}$. The reader will not find it surprising to learn, nor hard to prove, that this is compatible with the energy co-filtration. (Both are finite co-dimensional, and the rotation action stabilizes the Schubert cells.) In other words,

$$
\hat{\mathscr{H}}_{E}(x) \cong \lim \left(\mathscr{H}_{E} / \mathscr{H}_{E}^{\mathscr{C}}\right)
$$

with the corresponding topologies. This also shows that the group $G\left(\mathscr{M}_{x}\right)$ acts continuously on $\hat{\mathscr{H}}_{E}(x)$.

Note. As a matter of fact, the restrictions $\mathscr{H}_{E} / \mathscr{H}_{E}^{\mathscr{C}}$ to the Schubert cells $\mathscr{C}$ are isomorphic to the spaces of all sections of $\mathbf{E}(x) \otimes \mathscr{L}^{\otimes h}$ over the corresponding closed varieties, but this is not obvious. It follows essentially from Kumar's results $[\mathrm{Ku}]$, which show that the restriction of holomorphic sections from larger to smaller closed Schubert cells is surjective.

Choose now $n$ points $z_{1}, \ldots, z_{n} \in \mathbb{C}$, and assign to each an irreducible finitedimensional representation $V_{k}$ of $\mathfrak{g}_{\mathbb{C}}$. The tensor product $\hat{\mathscr{H}} \otimes \vec{V}(\vec{z})$ belongs to the category $\mathscr{C}\left(\mathfrak{g}_{\mathbb{C}[z]}, G\right)$ of $\mathfrak{g}_{\mathbb{C}[z]}$-modules which are direct products of $\mathfrak{g}$-isotypical components of finite-dimensional type. (A vector in a $G$-isotypical component of "large" type must only have large energy modes because the energy eigenspaces are finitedimensional.) As explained in Appendix A, the relative Lie algebra cohomology

$$
H^{*}\left(\mathfrak{g}_{\mathbb{C}[z]}, \mathfrak{g}_{\mathbb{C}} ; \hat{\mathscr{H}} \otimes \vec{V}(\vec{z})\right)
$$

as a derived functor on $\mathscr{C}\left(\mathfrak{g}_{\mathbb{C}[z]}, G\right)$ is then calculated by the standard complex

$$
\mathscr{C}^{q}\left(\mathfrak{g}_{\mathbb{C}[z]}, \mathfrak{g}_{\mathbb{C}} ; \hat{\mathscr{H}} \otimes \vec{V}(\vec{z})\right):=\operatorname{Hom}_{\mathfrak{g}}\left(\Lambda^{q}\left(\mathfrak{g}_{\mathbb{C}[z]} / \mathfrak{g}_{\mathbb{C}}\right) ; \hat{\mathscr{H}} \otimes \vec{V}(\vec{z})\right) .
$$

There is a natural finite co-dimensional co-filtration on $\mathscr{C}^{q}$, coming from the degree filtration on $\mathbb{C}[z]$ and the energy co-filtration on $\hat{\mathscr{H}}$. We shall write $\hat{\mathscr{C}}^{q}$ when thinking of $\mathscr{C}^{q}$ together with the inverse limit topology defined by this co-filtration.

\footnotetext{
17 Unlike the energy grading, this co-filtration is independent of the local coordinate.

18 One can see this by realizing $\Delta$ as the unit disk in $\mathbb{P}^{1}$ and using the factorization theorem [P-S, Ch.8]. which allows one to write a loop $\lambda \in G\left(\mathscr{M}_{1}\right)$ as $\lambda^{-} \cdot \chi \cdot \lambda^{+}$, where $\lambda^{+} \in \mathcal{O}_{1}, \lambda^{-}$is holomorphic on $\mathbb{P}^{1}-[0]$ and $\chi: \mathbb{C}^{\times} \rightarrow G_{\mathbb{C}}$ is a group homomorphism. It follows that $\lambda^{-}$is in fact algebraic, and since $\lambda \sim \lambda^{-} \cdot \chi$ in $X^{\text {alg }}, \lambda$ falls in some Schubert cell.
} 
The coboundary operator $\bar{\partial}$, given by formula $(2.1 .3)$ in Ch. 1 , is continuous on $\hat{\mathscr{C}}^{*}$ (it does not increase the energy). Because $\hat{\mathscr{C}}^{*}$ is an inverse limit of a system of finite-dimensional spaces, $\bar{\partial}$ also has closed range.

We summarize our conclusions in a proposition.

\section{Proposition 3.1.5.}

(i) The formally completed space $\hat{\mathscr{H}}_{E}(x)$ is a continuous representation of the Lie algebra $\mathrm{g}\left(\mathscr{M}_{x}\right)$ and of the group $G\left(\mathscr{M}_{x}\right)$;

(ii) It can be realized as the space of holomorphic sections $H^{0}\left(G\left(\mathscr{M}_{x}\right) / G\left(\mathcal{O}_{x}\right)\right.$; $\left.\mathbf{E}(x) \otimes \mathscr{L}^{\otimes h}\right)$, with the Schubert cell co-filtration;

(iii) $\hat{\mathscr{H}}:=\hat{\mathscr{H}}_{E}(\infty)$ and $\hat{\mathscr{H}} \otimes \vec{V}(\vec{z})$ are in the category $\mathscr{C}\left(\mathfrak{g}_{\mathbb{C}[z]}, G\right)$;

(iv) The cohomology groups $H^{*}\left(\mathfrak{g}_{\mathbb{C}[z]}, \mathfrak{g}_{\mathbb{C}} ; \hat{\mathscr{H}} \otimes \vec{V}(\vec{z})\right)$ are computed by the standard complex (3.1.4). The $\bar{\partial}$-operator is continuous on $\hat{\mathscr{C}}^{q}$, with closed range.

We append a technical remark which shall be used in Sect. 3.5:

(v) Let $\mathfrak{s}$ be the subalgebra of $L \mathfrak{g}_{\mathbb{C}}$, isomorphic to $\mathfrak{s l}_{2}(\mathbb{C})$, spanned by $\left\{e_{\alpha_{\max }}(-1)\right.$, $\left.h_{\alpha_{\max }}(0), e_{-\alpha_{\max }}(1)\right\}$. Then, $\hat{\mathscr{H}}$ is $\mathfrak{s}$-integrable, and is the direct product of it $\mathfrak{s}$-isotypical components.

Proof of $(v)$. We must show that vectors in an $\mathfrak{s}$-isotypical component of "large" type must only have energy components of large (negative) energy. Consider the modified energy operator $\tilde{E}:=E+\frac{1}{2} h_{x_{\max }}(0)$. Because $E$ and $h_{x_{\max }}(0)$ can be simultaneously diagonalized, we can see that

(a) $\tilde{E}$ commutes with $\mathfrak{s}$ and acts semisimply on $\hat{\mathscr{H}}$, with integral or half-integral eigenvalues. Now, on an $E$-eigenspace, $h_{\alpha_{\max }}(0)$ is, roughly, bounded by the square root $^{19}$ of $(-E)$. It follows that

(b) the eigenvalues of $\tilde{E}$ are bounded above and have finite multiplicity, and also

(c) an $\tilde{E}$-eigenvector for a large (negative) eigenvalue will only project to $E$ eigencomponents of large (negative) $E$-value.

By the finiteness property (b), vectors of large $\mathfrak{s}$-type, which by (a) we may assume to be $\tilde{E}$-eigenvectors, can only appear at the large (negative) $\tilde{E}$ eigenvalues. By (b), they are combinations of large-eigenvalue $E$-eigenvectors.

3.2. A Generalized Bernstein-Gelfand-Gelfand Resolution of $\mathscr{H} \otimes \vec{V}(\vec{z})$. In this section we describe a "generalized BGG" resolution of $\mathscr{H}$, and of the twisted representation $\mathscr{H} \otimes \vec{V}(\vec{z})$, by injective $\left(\mathfrak{g}_{\mathbb{C}[z]}, G\right)$-modules. Such resolutions for highest weight representations of Kac-Moody algebras are a well-known tool in cohomology calculations $[\mathrm{RC}-\mathrm{W}]$. They reduce the calculation of $H^{*}\left(\mathfrak{g}_{\mathbb{C}[z]}, \mathfrak{g}_{\mathbb{C}} ; \mathscr{H} \otimes \vec{V}(\vec{z})\right)$ to a finite-dimensional problem. In the one-point case, the BGG resolution determines the cohomology completely (Corollary 3.2.7); but in the case of several points, a direct solution of this finite-dimensional problem is not apparent ${ }^{20}$, and the only proof of Theorem 3.1.3 that I know is the rather lengthy Hilbert space argument of Sect. 2 and Sect. 3.3-3.5. It should be noted that a direct proof of vanishing of

19 The Segal-Sugawara formula expresses $L_{0}$, which differs from $(-E)$ by a constant, as a sum of non-negative operators, including $-\frac{1}{2(h+\mathbb{C})} \cdot h_{\alpha_{\max }}(0)^{2}$.

20 Except for the simple case of $\mathfrak{g}=\mathfrak{s u}(2)$. 
higher cohomology in the complex (3.2.7) would give, together with Sect. 3.6, an elementary determination of the fusion rules.

The basic central extension of the Lie algebra $g\left(\mathscr{M}_{x}\right)$ can be used to define, for every $h$, the "universal enveloping algebra at level $h$,"

$$
\begin{aligned}
U_{k}\left(\mathfrak{g} \mathscr{M}_{x}\right):= & \text { tensor algebra of } \mathfrak{g}\left(\mathscr{M}_{x}\right), \text { modulo the relations } \\
& \{\xi \otimes \eta-\eta \otimes \xi=[\xi, \eta]+h \cdot \omega(\xi, \eta)\} .
\end{aligned}
$$

Here, $\omega(\xi, \eta)=\frac{1}{2 \pi} \int_{S} \xi \cdot d \eta$ is the Lie algebra cocycle defining the basic central extension. The extension can be trivialized uniquely over $\mathfrak{g}\left(\mathcal{O}_{\infty}\right)$ and $\mathfrak{g}_{\mathbb{C}[z]}$, leading to well-defined inclusions $U\left(\mathfrak{g}_{\mathbb{C}[z]}\right) \subset U_{h}\left(\mathfrak{g}\left(\mathscr{M}_{\infty}\right)\right)$ and $U\left(\mathfrak{g}\left(\mathcal{O}_{\infty}\right)\right) \subset U_{h}\left(\mathfrak{g}\left(\mathscr{M}_{\infty}\right)\right)$. Since a loop $\lambda \in \mathfrak{g}\left(\mathscr{M}_{\infty}\right)$ can be split as $\lambda=\lambda^{+}+\lambda^{-}$, with $\lambda^{+} \in \mathfrak{g}_{\mathbb{C}[z]}$ and $\lambda^{-} \in$ $\mathfrak{g}\left(\mathcal{O}_{\infty}\right)$, uniquely up to a constant, this implies the following, as an easy application of the Poincaré-Birkhoff-Witt theorem.

Lemma 3.2.1. As a left $U\left(\mathfrak{g}_{\mathbb{C}[z]}\right)$-module and right $U\left(\mathfrak{g}\left(\mathcal{O}_{\infty}\right)\right)$-module,

$$
U_{h}\left(\mathfrak{g}\left(\mathscr{M}_{\infty}\right)\right) \cong U\left(\mathfrak{g}_{\mathbb{C}[z]}\right) \underset{U\left(\mathfrak{g}_{\mathbb{C}}\right)}{\bigotimes} U\left(\mathfrak{g}\left(\mathcal{O}_{\infty}\right)\right)
$$

Let for now $F$ be any representation of $G$ and $x$ a point in $\mathbb{P}^{1}$. The space

$$
\Gamma_{F}(x):=\operatorname{Hom}_{\mathfrak{g}\left(\mathcal{G}_{x}\right)}\left(U_{h}\left(\mathfrak{g}\left(\mathscr{M}_{x}\right)\right) ; F(x)\right),
$$

where the subscript $\mathfrak{g}\left(\mathcal{O}_{x}\right)$ indicates the $\mathfrak{g}\left(\mathcal{O}_{x}\right)$-equivariant linear homomorphisms for the negative right multiplication action on $U_{h}\left(g\left(\mathscr{M}_{x}\right)\right)$ and the obvious action on $F=F(x)$, is naturally a $\left(\mathfrak{g}\left(\mathscr{M}_{x}\right), G\right)$-module. We shall only use the case $x=\infty$ and write $\Gamma_{F}$ for $\Gamma_{F}(\infty) ; \Gamma_{F}$ is, in particular a $\left(\mathfrak{g}_{\mathbb{C}[z]}, G\right)$-module. As a consequence of Lemma 3.2.1, we have

Lemma 3.2.2. $\Gamma_{F}$ is an injective $\left(\mathfrak{g}_{\mathbb{C}[z]}, G\right)$-module.

Proof. We have to show that $M \mapsto \operatorname{Hom}_{\mathfrak{g}_{\mathbb{C}[z]}}\left(M ; \Gamma_{F}\right)$ is an exact functor on the category $\mathscr{C}\left(\mathfrak{g}_{\mathbb{C}[z]}, G\right)$. From Lemma 3.2 .1 , it follows that, as left $U\left(\mathfrak{g}_{\mathbb{C}[z]}\right)$-modules,

$$
\Gamma_{F} \cong \operatorname{Hom}_{\mathfrak{g}_{\mathbb{C}}}\left(U\left(\mathfrak{g}_{\mathbb{C}[z]}\right) ; W\right)
$$

But for a $\left(\mathfrak{g}_{\mathbb{C}[z]}, G\right)$-module $M$, we have

$$
\begin{aligned}
\operatorname{Hom}_{\mathfrak{G}_{\mathbb{C}[z]}}\left(M ; \Gamma_{F}\right) & \cong \operatorname{Hom}_{\mathfrak{G}_{\mathbb{C}[z]}}\left(M ; \operatorname{Hom}_{\mathfrak{g}_{\mathbb{C}}}\left(U\left(\mathfrak{g}_{\mathbb{C}[z]}\right) ; F\right)\right) \\
& \cong \operatorname{Hom}_{\mathfrak{g}_{\mathbb{C}}}\left(M \underset{U\left(\mathfrak{g}_{\mathbb{C}[z]}\right)}{\otimes} U\left(\mathfrak{g}_{\mathbb{C}[z]}\right) ; F\right) \cong \operatorname{Hom}_{\mathfrak{g}_{\mathbb{C}}}(M ; F),
\end{aligned}
$$

and $M \mapsto \operatorname{Hom}_{\mathfrak{g}_{\mathbb{C}}}(M ; F)$ is an exact functor on $\mathscr{C}\left(\mathfrak{g}_{\mathbb{C}[z]}, G\right)$.

Corollary 3.2.4. With the tensor product action, $\Gamma_{F} \otimes V_{1}\left(z_{1}\right) \otimes \cdots \otimes V_{n}\left(z_{n}\right)$ is an injective $\left(\mathfrak{g}_{\mathbb{C}[z]}, G\right)$-module.

Proof. Identical to (3.2.2).

Let $K(F ; h)$ denote the set of $G$-representations which are affine-conjugate to $F$ at level $h$ (relative to the $(h+c)$-action of the affine Weyl group on the weight lattice of $\mathfrak{g}$ ). 
Proposition 3.2.5. (BGG resolution in $\left.\mathscr{C}\left(\mathfrak{g}_{\mathbb{C}|z|}, G\right)\right)$. Let $F \in R(G)$ be regular antidominant. There exists a $\mathscr{C}\left(\mathfrak{g}_{\mathbb{C}[z]}, G\right)$-injective resolution of $\mathscr{H}_{F}$, in which the maps are $\mathrm{g}\left(\mathscr{M}_{\infty}\right)$-module homomorphisms:

$$
\hat{\mathscr{H}}_{F} \rightarrow \Gamma_{F} \rightarrow \bigoplus_{\substack{F^{\prime} \in K(F ; h) \\\left(F^{\prime}\right)=1}} \Gamma_{F} \rightarrow \bigotimes_{\substack{F^{\prime} \in K(F, h) \\\left(F^{\prime}\right)=2}} \Gamma_{F} \rightarrow \bigotimes_{\substack{F^{\prime} \in K(F, h) \\\left(F^{\prime}\right)=3}} \Gamma_{F} \rightarrow \ldots
$$

Proof. See [RC-W]. Sect. 8, for the proof of the dual statement.

By tensoring (3.2.5) with $\vec{V}(\vec{z})=V_{1}\left(z_{1}\right) \otimes \cdots \otimes V_{n}\left(z_{n}\right)$, we obtain

Corollary 3.2.6. The following is an injective resolution of $\hat{\mathscr{H}}_{W} \otimes \vec{V}(\vec{z})$ in $\mathscr{C}\left(\mathfrak{g}_{\mathbb{C}[z]}, G\right)$ :

$$
\hat{\mathscr{H}}_{F} \rightarrow \Gamma_{F} \otimes \vec{V}(\vec{z}) \rightarrow \bigoplus_{\substack{F^{\prime} \in K(F, h) \\\left(\left(F^{\prime}\right)=1\right.}} \Gamma_{F} \otimes \vec{V}(\vec{z}) \rightarrow \bigoplus_{\substack{F^{\prime} \in K(F ; h) \\ \ell\left(F^{\prime}\right)=2}} \Gamma_{F} \otimes \vec{V}(\vec{z}) \rightarrow \ldots
$$

Corollary 3.2.7. (i) The relative Lie algebra cohomology $H^{*}\left(\mathfrak{g}_{\mathbb{C}[z]}, \mathfrak{g}_{\mathbb{C}} ; \hat{\mathscr{H}} \otimes \vec{V}(\vec{z})\right)$ is the cohomology of a finite-dimensional complex $K^{*}$, where

$$
K^{q}=\bigoplus_{\substack{F^{\prime} \in K(F ; h) \\ f\left(F^{\prime}\right)=q}}\left(W^{\prime} \otimes V_{1} \otimes \cdots \otimes V_{n}\right)^{\mathfrak{g}_{\mathbb{C}}}
$$

and the coboundary maps are polynomial functions of the $z_{k}{ }^{\prime}$.

The Euler characteristic is thus independent of the points $z_{k}$, whether they are distinct or not.

(ii) In particular, for $n=1, H^{q}\left(\mathfrak{g}_{\mathbb{C}[z]}, \mathfrak{g}_{\mathbb{C}} ; \hat{\mathscr{H}} \otimes V_{1}\left(z_{1}\right)\right)=0$ for all $q$ unless $V_{1}$ is affine-conjugate to the representation $F^{t}$ dual to $F$, in which case

$$
H^{q}\left(\mathfrak{g}_{\mathbb{C}[z]}, \mathfrak{g}_{\mathbb{C}} ; \hat{\mathscr{H}} \otimes V_{1}\left(z_{1}\right)\right)=\mathbb{C} \text { for } q=\ell\left(V_{1}\right),
$$

and

$$
H^{q}\left(\mathfrak{g}_{\mathbb{C}[z]}, \mathfrak{g}_{\mathbb{C}} ; \hat{\mathscr{H}} \otimes V_{1}\left(z_{1}\right)\right)=0 \text { for all other } q .
$$

Proof. Part (i) follows from the last corollary and the fact that

$$
\begin{aligned}
\left(\Gamma_{F} \otimes V_{1}\left(z_{1}\right) \otimes \cdots \otimes V_{n}\left(z_{n}\right)\right)^{\mathfrak{g}_{\mathbb{C}[z]}} & \cong\left(\operatorname{Hom}_{\mathfrak{g}_{\mathbb{C}}}\left(U\left(\mathfrak{g}_{\mathbb{C}[z]}\right) ; F^{\prime}\right)\right. \\
& \otimes V_{1}\left(z_{1}\right) \otimes \cdots \otimes V_{n}\left(z_{n}\right)^{\mathfrak{g}_{\mathbb{C}[z]}} \\
& \cong\left(F^{\prime} \otimes V_{1} \otimes \cdots \otimes V_{n}\right)^{\mathfrak{g}_{\mathbb{C}}} .
\end{aligned}
$$

One could produce an explicit formula for the coboundary maps in (3.2.5) and use it to prove that the maps in Corollary 3.2.7 are polynomial in the $z_{k}$ 's. In truth, the only relevant property of the $\partial$ 's is that they give a finite energy shift: the corresponding maps in (3.2.7) will then have a polynomial dependence in the $z_{k}$ 's, of total degree no more than this energy shift. However, we know that after suitable normalization, the energy on the $\Gamma_{F}$ is given by the Segal-Sugawara operator $L_{0}$; it will therefore be preserved by the coboundary maps (3.2.5), which are $L \mathfrak{g}$-homomorphisms.

3.3. Hilbert Space Representative. For the benefit of the reader, this paper's basic policy is to relegate unpleasant formulae to the Appendixes. We have made an exception for Proposition 3.3.1, proved below. It says that every formal cohomology 
class has a representative in the Hilbert space domain of the $\bar{\partial}$-operator, and provides the link with the Hilbert space theory in Sect. 2.

We have proved (Theorem 2.2.3) that, if the $V_{k}$ 's are antidominant and if the points $z_{k}$ are far apart, then, in the Hilbert space complex $\mathscr{C}_{\mathrm{H} 1 \mathrm{lb}}^{*}$ of $(2.1 .3)$, the range of $\bar{\partial}$ is dense in its kernel in positive degrees. We shall deduce from here, at first the same assumptions, the vanishing of higher cohomology in the formally completed complex $\hat{\mathscr{C}}^{*}$. Regarding the complex $\hat{\mathscr{C}}^{*}$ together with its inverse limit topology described in Sect. 3.1, recall from 3.1.5 that

(i) On $\hat{\mathscr{C}}^{*}$, the operator $\bar{\partial}$ is continuous, with closed range;

(ii) The inclusion $\mathscr{C}_{\text {Hilb }}^{*} \subset \hat{\mathscr{C}}^{*}$ is continuous.

If a positive degree cohomology class in $\hat{\mathscr{C}}^{*}$ has a representative $\phi$ which happens to lie in the Hilbert space domain of $\bar{\partial}$, then by Theorem $(2.2 .3), \phi$ lies in the $\mathscr{C}_{\text {Hilb }}^{*}$-closure of the Hillbert space range of $\bar{\partial}$. By (ii), $\phi$ then lies in the $\hat{\mathscr{C}}^{*}$-closure of the $\hat{\mathscr{C}}^{*}$-range of $\bar{\partial}$, which according to (i) is the same as the $\hat{\mathscr{C}}^{*}$-range; but then, $\phi$ represents 0 in cohomology. We therefore get a vanishing theorem in $\hat{\mathscr{C}}^{*}$ from the following proposition:

Proposition 3.3.1. (Hilbert Space Representative). As long as the points $z_{k}$ are inside the unit disk, every cohomology class in $\hat{\mathscr{C}}^{*}$ has a representative in the Hilbert space domain of $\bar{\partial}$. In fact, there exists a representative $\psi$ whose energy components $\psi(N)$ satisfy

$$
\|\psi(N)\| \leqq o\left(r^{|N|}\right), \text { and }\|\bar{\partial}(\psi(N))\| \leqq o\left(r^{|N|}\right)
$$

for any $r>\mu=\max \left\{\left|z_{k}\right|\right\}$. (Here, $\psi$ may be a zero-form, and the representations $V_{k}$ are unrestricted.)

Proof. We must make use of a new Hilbert space norm \|\|$_{\text {new }}$, finer than the one defined in Sect. 2.1. Let $\mathfrak{g}_{+}$be the Lie subalgebra of positive modes in $\mathfrak{g}_{\mathbb{C}[z]}$. We obtain the new norm from the old one by replacing the $H_{1 / 2}$ inner product on $\mathfrak{g}_{+}$with the straight $L^{2}$ norm, while the hermitian structures on $\mathscr{H}$ and the $V_{k}$ 's are kept unchanged. Thus, for the functional $\xi^{a}(-n) \in\left(\mathfrak{g}_{+}\right)^{t}$, dual to $\xi_{a}(n)$ in the Fourier mode basis of $\mathfrak{g}_{+}$, we have $\left\|\xi^{a}(-n)\right\|_{\text {new }}^{2}=1$, while $\left\|\xi^{a}(-n)\right\|^{2}=1 / n$. Since \|\|$\leqq\|\|_{\text {new }}$, it suffices to prove (3.3.1) for \|\|$_{\text {new }}$.

Pick a form $\psi \in \operatorname{Ker}(\bar{\partial}) \subset \hat{\mathscr{C}}^{*}$. To avoid spurious "_" signs in subscripts, we change the sign of the energy grading to positive for the remainder of this section. Recall from (2.3.1) and (2.3.2) that $\bar{\partial}=\bar{\partial}_{0}+\cdots+\bar{\partial}_{N}+\ldots$, with $\left|\bar{\partial}_{N}\right|=O\left(\mu^{N}\right)$. This, incidentally, is true both in the old and the new Hilbert space norm. Decomposing $\psi$ as $\psi=\psi_{1}+\cdots+\psi_{N}+\cdots$, we get

$$
\begin{aligned}
& \bar{\partial}_{0} \psi_{0}=0, \\
& \bar{\partial}_{0} \psi_{1}=-\bar{\partial}_{1} \psi_{0}, \\
& \cdots \\
& \bar{\partial}_{0} \psi_{N}=-\bar{\partial}_{1} \psi_{N-1}-\cdots-\bar{\partial}_{N-1} \psi_{1}-\bar{\partial}_{N} \psi_{0}, \\
& \cdots \cdots
\end{aligned}
$$


Thus

$$
\begin{aligned}
& \left\|\bar{\partial}_{0} \psi_{N}\right\|_{\text {new }} \leqq\left\|\bar{\partial}_{1} \psi_{N-1}\right\|_{\text {new }+}+\cdots+\left\|\bar{\partial}_{N-1} \psi_{2}\right\|_{\text {new }}+\left\|\bar{\partial}_{N} \psi_{0}\right\|_{\text {new }} \\
& \quad \leqq C \cdot \mu \cdot\left\|\psi_{N-1}\right\|_{\text {new }+}+\cdots+C \cdot \mu^{N-1} \cdot\left\|\psi_{1}\right\|_{\text {new }}+C \cdot \mu^{N} \cdot\left\|\psi_{0}\right\|_{\text {new }} \cdot
\end{aligned}
$$

Now we replace $\psi$ by a representative of its class which lies in the kernel of $\bar{\partial}_{0 \text {, new }}^{*}$. (This can be done recursively while preserving all the relations (3.3.2): at every $k, \psi_{k}+\bar{\partial}_{0} \chi_{k} \in \operatorname{Ker}\left(\bar{\partial}_{0}^{*}\right)$ for some $\chi_{k}$ of energy $k$, but then replacing $\psi$ by $\psi+\bar{\partial} \chi$ keeps it in $\operatorname{Ker}(\bar{\partial})$, does not change its class, and only affects components in energy greater than $k$.) For such a $\psi$,

$$
\left\|\bar{\partial}_{0} \psi_{N}\right\|_{\text {new }}=\left(\left\|\bar{\partial}_{0} \psi_{N}\right\|_{\text {new }}^{2}+\left\|\bar{\partial}_{0, \text { new }}^{*} \psi_{N}\right\|_{\text {new }}^{2}\right)^{1 / 2}=\left\langle\psi_{N}\left|\bar{\square}_{0, \text { new }}\right| \psi_{N}\right\rangle^{1 / 2}
$$

The most we can say about the "old" $\bar{\partial}_{0}$-Laplacian $\bar{\square}_{0}$ is that its essential spectrum is bounded away from zero (and even that is rather difficult to prove). But the Laplacian $\bar{\square}_{0 \text {,new }}$ is much better behaved: it is, essentially, the energy operator on $\hat{\mathscr{C}}^{*}$, and has discrete, finit-multiplicity spectrum. More precisely, let $E$ be the (positive) energy operator on the factors $\mathscr{H}$ and $\Lambda^{*}$. Let also $c_{0}$ be the eigenvalue of the Casimir operator of $\mathfrak{g}$ on the ground states of $\mathscr{H}$, and denote by $\Delta_{\vec{V}}$ the Casimir operator acting on $\vec{V}=V_{1} \otimes \cdots \otimes V_{n}$. We have (cf. Appendix B.2, Prop. B.2.5)

Lemma 3.3.4. On the Hilbert space complex $\operatorname{Hom}_{\mathfrak{g}_{\mathbb{C}}}\left(\Lambda^{*}\left(\mathfrak{g}_{+}\right) ; \mathscr{H} \otimes \vec{V}\right)$, we have

$$
\bar{\square}_{0, \text { new }}=(h+c) \cdot E+c_{0}-\Delta_{\vec{V}},
$$

and thus

$$
(h+c) \cdot E+A \leqq \bar{\square}_{0, \text { new }} \leqq(h+c) \cdot E+B
$$

with the constants $A$ and $B$ depending only on the representations $V_{k}$.

Combining (3.3.3) and (3.3.4), we obtain that, for large $N$,

$$
\left\|\psi_{N}\right\|_{\text {new }} \leqq \frac{K}{\sqrt{N}} \sum_{p=0}^{N-1} \mu^{N-p} \cdot\left\|\psi_{p}\right\|_{\text {new }}
$$

or, setting $x_{p}=\left\|\psi_{p}\right\|_{\text {new }} \cdot \mu^{-p}$,

$$
x_{N} \leqq \frac{K}{\sqrt{N}} \sum_{p=0}^{N-1} x_{p}
$$

whence

$$
\sum_{p=0}^{N-1} x_{p} \leqq\left(1+\frac{K}{\sqrt{N}}\right) \sum_{p=0}^{N-1} x_{p}
$$

From here, it follows that, for some constant $K^{\prime}$,

$$
\sum_{p=0}^{N} x_{p} \leqq \prod_{m=N_{0}}^{N}\left(1+\frac{K}{\sqrt{m}}\right) \cdot \sum_{p=0}^{N_{0}} x_{p} \leqq K^{\prime} \cdot \exp (2 K \sqrt{N}),
$$


thus

$$
x_{N} \leqq K^{\prime} \cdot \exp (2 K \sqrt{N})
$$

and

$$
\left\|\psi_{N}\right\|_{\text {new }} \leqq K^{\prime} \cdot \exp (2 K \sqrt{N}) \cdot \mu^{N}=o\left(r^{N}\right) \text { for } r>\mu,
$$

as promised.

The second inequality in (3.3.4) follows from the upper bound for $\bar{\square}_{0 \text {, new }}$ in (3.3.1).

To summarize, Chapter 2 and Proposition 3.3.1 together imply

Proposition 3.3.5. If the representations $V_{k}$ are antidominant and the points $z_{k}$ are inside the unit disk and near the boundary, but not near each other, then $H^{q}\left(\mathfrak{g}_{\mathbb{C}[z]}, \mathfrak{g}_{\mathbb{C}} ; \hat{\mathscr{H}} \otimes V(\vec{z})\right)=0$ for $q>0$.

3.4. The Knizhnik-Zamolodchikov Connection. Now that any reference to Hilbert space has been removed, the restriction in (3.3.5) on the points $z_{k}$ makes little sense, and we would like to replace it with the condition that the points should be distinct. The idea is to move the points $z_{k}$ along the flow-lines of a holomorphic vector field. The Lie algebra of polynomial vector fields on $\mathbb{C}$ acts naturally on $\mathfrak{g}_{\mathbb{C}[z]}$ by infinitesimal automorphism, and there exists an interwining action on $\hat{\mathscr{H}}$, given by the Segal-Sugawara operators [P-S]; but these actions cannot be exponentiated ${ }^{21}$. It turns out, however, that the induced action of a vector field $\phi$ on the cohomology groups $H^{*}\left(\mathfrak{g}_{\mathbb{C}[z]}, \mathfrak{g}_{\mathbb{C}} ; \hat{\mathscr{H}} \otimes \vec{V}(\vec{z})\right)$ can be exponentiated to give isomorphisms

$$
\left(\Phi_{t}\right)_{*}: H^{*}\left(\mathfrak{g}_{\mathbb{C}[z]}, \mathfrak{g}_{\mathbb{C}} ; \hat{\mathscr{H}} \otimes \vec{V}(\vec{z})\right) \stackrel{\cong}{\rightrightarrows} H^{*}\left(\mathfrak{g}_{\mathbb{C}[z]}, \mathfrak{g}_{\mathbb{C}} ; \hat{\mathscr{H}} \otimes \vec{V}\left(\Phi_{t}(\vec{z})\right)\right)
$$

where $\Phi_{t}$ denotes the flow along $\phi$ at time $t$, and $\Phi_{t}(\vec{z})$ is the $n$-tuple $\left(\Phi_{t}\left(z_{t}\right), \ldots, \Phi_{t}\right.$ $\left(z_{n}\right)$ ) of images $\Phi_{t}\left(z_{k}\right)$ of the points $z_{k}$. All this holds assuming that $t$ is smaller than any of the exit times of the points $z_{k}$ under $\phi$.

This all follows from the finite-dimensional presentation (3.2.7) of the spaces $H^{*}\left(\mathfrak{g}_{\mathbb{C}[z]}, \mathfrak{g}_{\mathbb{C}} ; \hat{\mathscr{H}} \otimes \vec{V}(\vec{z})\right)$; the coboundary operators in the complex $(3.2 .5)$ are $L \mathfrak{g}$ module homomorphisms, and so they commute with the Virasoro action. The action can be restricted to the finite-dimensional complex (3.2.7), where it must exponentiate to give (3.4.1). To find the inverse, one just exponentiates $(-\phi)$.

Less obvious and more interesting is the fact that, after a slight modification, the isomorphisms (3.4.1) will be unique, up to monodromy, on the configuration space $Z(n)$ of $n$ distinct ordered points in $\mathbb{C}$. Since this is not a necessary part of the proof, we pause for a moment to summarize the present state of the argument before digressing any further on this topic.

\section{Proposition 3.4.2.}

(0) The Lie algebra cohomology $H^{*}\left(\mathfrak{g}_{\mathbb{C}[z]}, \mathfrak{g}_{\mathbb{C}} ; \hat{\mathscr{H}} \otimes \vec{V}(\vec{z})\right)$ is finite-dimensional. Its dimension is independent of the points $z_{k}$, as long as they stay distinct.

(1) The Euler characteristic $\chi\left(\mathfrak{g}_{\mathbb{C}[z]}, \mathfrak{g}_{\mathbb{C}} ; \hat{\mathscr{H}} \otimes \vec{V}(\vec{z})\right)$ is independent of the points $z_{k} \in \mathbb{C}$, distinct or not.

21 We can exponentiate the actions of $L_{0}$ and $L_{1}$, leading to affine coordinate changes on $\mathbb{C}$, but this is not sufficient if more than two points are to be moved. 
(2) In the one-point case, $H^{*}\left(\mathfrak{g}_{\mathbb{C}[z]}, \mathfrak{g}_{\mathbb{C}} ; \hat{\mathscr{H}} \otimes V(0)\right)=0$, except when $\hat{\mathscr{H}}$ is induced from $V^{t}$; in that case, $H^{q} \cong \mathbb{C}$ in degree $q=\ell(V)$, and $H^{q} \cong 0$ in all other degrees.

(3) If the points are distinct and all the factors $V_{k}$ are antidominant (i.e. $\left.\lambda_{k} \cdot \alpha_{\max } \leqq h+1\right)$, all higher cohomology vanishes.

Parts (1), (2) and the first statement in (0) follow from Corollary 3.2.7. The second statement in $(0)$ follows from the preceding remarks. Part (3) is Proposition 3.3.8 plus Part $(0)$.

We will finish the proof of Theorem 0 in the next section. The remainder of this section discusses the Knizhnik-Zamolodchikov connection on the bundle of spaces $H^{*}\left(\mathfrak{g}_{\mathbb{C}[z]}, \mathfrak{g}_{\mathbb{C}} ; \hat{\mathscr{H}} \otimes \vec{V}(\vec{z})\right)$ over $Z(n)$. It is not a necessary part of the proof, and the reader may skip ahead to Sect. 3.5.

Consider the complex of trivial vector bundles over $\mathbb{C}^{\prime \prime}$, coming from Corollary 3.2.7:

$$
K^{q}=\bigoplus_{\substack{F \in K(E, h) \\(F)=q}}\left(F \otimes V_{1} \otimes \cdots \otimes V_{n}\right)^{\mathfrak{g}}
$$

As noted there, the coboundary operators are polynomial functions on $\mathbb{C}^{n}$. The

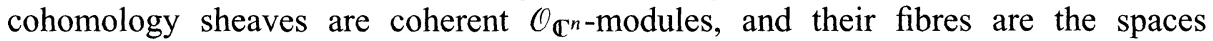
$H^{*}\left(\mathfrak{g}_{\mathbb{C}[z]}, \mathfrak{g}_{\mathbb{C}} ; \hat{\mathscr{H}} \otimes \vec{V}(\vec{z})\right)$. Now, the fibre of $K^{q}$ at a point $\left(z_{1}, \ldots, z_{n}\right)$ is naturally isomorphic to a corresponding sum of terms $\left(\Gamma_{F} \otimes \vec{V}(\vec{z})\right)^{\mathfrak{g}_{\mathbb{C}[z]}}$ in (3.2.6). A polynomial vector field $\phi=\sum_{p=0}^{N} \phi_{p} z^{p} \frac{\partial}{\partial z}$ on $\mathbb{C}$ gives rise to the field $\phi_{Z}=\sum_{k=1}^{p} \phi\left(z_{k}\right) \frac{\partial}{\partial z_{k}}$ on $\mathbb{C}^{n}$. We explicitly lift the action of $\phi_{Z}$ to the bundles $\left(\Gamma_{F} \otimes \vec{V}(\vec{z})\right)^{\mathfrak{9} \mathbb{C}[z]}$, and then prove that, when restricted to $Z(n)$, the liftings come from a flat connection.

Recall the standard Segal-Sugawara operators on the generalized Verma modules $\Gamma_{F}$ of Sect. 3.2:

$$
L_{p}=\frac{1}{2(h+c)} \sum_{-\infty \leqq m \leqq \infty}: \xi_{a}(p-m) \xi_{a}(m):
$$

Their action commutes with the coboundary operators in the complexes (3.2.5) (and (3.2.6)), since the latter are $L \mathfrak{g}_{\mathbb{C}}$-equivariant. Define

$$
\tilde{\phi}=\sum_{k=1}^{n} \phi\left(z_{k}\right) \frac{\partial}{\partial z_{k}}+\sum_{p=0}^{N} \phi_{p} \cdot L_{p-1}+\sum_{k=1}^{n} \phi^{\prime}\left(z_{k}\right) \frac{c_{k}}{h+c} .
$$

Here, the first term gives the trivial action of $\phi_{Z}$ on the trivial bundle $\Gamma_{F} \otimes$ $V_{1} \otimes \cdots \otimes V_{n}$ over $Z(n)$, the $L_{p}$ 's in the second sum act on the factor $\Gamma_{F}$, and the numbers $c_{k}$ are the eigenvalue of the Casimir operators on the $V_{k}$ 's.

Proposition 3.4.8. The lifting $\phi_{Z} \mapsto \tilde{\phi}$ determines a flat holomorphic connection over $Z(n)$ on the terms $\left(F \otimes V_{1} \otimes \cdots \otimes V_{n}\right)^{\mathfrak{g}}$ of the complex $K^{q}$, commuting with the coboundary operators. Specifically, $\tilde{\phi}$ is the lift of $\phi_{Z}$ by means of the $G$ invariant connection $\nabla$ on $V_{1} \otimes \cdots \otimes V_{n}$ given by

$$
\nabla=\sum_{k=1}^{n} \frac{\partial}{\partial z_{k}} \otimes d z_{k}+\frac{1}{2(h+c)} \cdot \sum_{\substack{\neq \neq k \\ a}} \frac{d z_{j}-d z_{k}}{z_{J}-z_{k}} \cdot \theta_{j}\left(\xi_{a}\right) \otimes \theta_{k}\left(\xi_{a}\right) .
$$


Proof. We already know that $\tilde{\phi}$ acts on the complex $\Gamma \otimes \otimes \vec{V}(\vec{z})$, preserving the coboundary operators. If $\tilde{R}(\xi)$ denotes the total action on $\Gamma_{F} \otimes \vec{V}(\vec{z})$ of a loop $\xi \in \mathfrak{g}_{\mathbb{C}[z]}$, one can check directly from $(3.4 .7)$ that $[\tilde{\phi}, \tilde{R}(\xi)]=\tilde{R}(\phi(\xi))$. It follows that $\tilde{\phi}$ also preserves the subspace of $\mathfrak{g}_{\mathbb{C}[z]}$-invariant sections. Since the map $\phi_{Z} \mapsto \tilde{\phi}$ gives a surjection of $\mathfrak{P}$ onto the tangent space to $Z(n)$ at any point, the connection in question is uniquely determined, if it exists. In that case, flatness follows from the fact that $[\tilde{\phi}, \tilde{\psi}]=\tilde{\chi}$, for $\chi=[\phi, \psi]$.

Finding $\tilde{\phi}$ explicitly on $\left(F \otimes V_{1} \otimes \cdots \otimes V_{n}\right)^{\mathfrak{g}}$ requires a moment's thought. To sum it up, because $F$ is the space of highest-energy vectors in $\Gamma_{F}$, the action of $L_{p}$ is obtained, first by dropping all the terms in (3.4.6) which contain negative modes, and then, since we are restricting to $\mathfrak{g}_{\mathbb{C}[z]}$-invariants, by evaluating the remainder on $V_{1}\left(z_{1}\right) \otimes \cdots \otimes V_{n}\left(z_{n}\right)$. The result is

$$
\frac{1}{2(h+c)} \sum_{j, k ; a} z_{j}^{m-p} \cdot z_{k}^{p} \cdot \theta_{j}\left(\xi_{a}\right) \otimes \theta_{k}\left(\xi_{a}\right)
$$

where $\theta_{k}$ designates the action of $\mathfrak{g}$ on $V_{k}$. We can rewrite it as

$$
\frac{1}{2(h+c)} \cdot \sum_{\substack{l \neq k \\ a}} \frac{z_{j}^{p+1}-z_{k}^{p+1}}{z_{j}-z_{k}} \cdot \theta_{j}\left(\xi_{a}\right) \otimes \theta_{k}\left(\xi_{a}\right)-\frac{1}{h+c} \cdot \sum_{k=1}^{n} \frac{\partial}{\partial z_{k}}\left(z_{k}^{p+1}\right) \cdot c_{k} .
$$

Summing over $p$ as in (3.4.7) and cancelling the last term gives

$$
\tilde{\phi}=\sum_{k=1}^{n} \phi\left(z_{k}\right) \frac{\partial}{\partial z_{k}}+\frac{1}{2(h+c)} \cdot \sum_{\substack{i \neq k \\ a}} \frac{\phi\left(z_{\jmath}\right)-\phi\left(z_{k}\right)}{z_{j}-z_{k}} \cdot \theta_{j}\left(\xi_{a}\right) \otimes \theta_{k}\left(\xi_{a}\right),
$$

and, as advertised, $\tilde{\phi}$ is the lifting to $\left(F \otimes V_{1} \otimes \cdots \otimes V_{n}\right)^{\mathfrak{g}}$ of $\phi_{z}$ via the connection 3.4.9 on $V_{1} \otimes \cdots \otimes V_{n}$. The latter is the standard form of the KnizhnikZamolodchikov connection.

3.5. Bott Reflection, and End of Proof of Theorem 0. We now proceed to the general case of Theorem 0 . The argument is borrowed from Bott's original proof [B] of the BWB theorem, and translated into Lie algebra cohomology; the basic step is the Reflection Lemma (3.5.5) below.

We reduce the calculation of $H^{*}\left(\mathfrak{g}_{\mathbb{C}[z]}, \mathfrak{g}_{\mathbb{C}} ; \mathscr{H} \otimes V_{1}\left(z_{1}\right) \otimes \cdots \otimes V_{n}\left(z_{n}\right)\right)$ to the case where all the $V_{k}$ 's are antidominant by working on each factor separately. Because the linear transformations $z \mapsto a z+b$ act on $\hat{\mathscr{H}}$, we may assume that $z_{1}=0$. (We also keep the assumptions of Theorem 0 that the $V_{k}$ are irreducible and the points $z_{k}$ are distinct.) Set $V_{1}=V, \lambda_{1}=\lambda$ and denote $\hat{\mathscr{H}} \otimes V_{2}\left(z_{2}\right) \otimes \cdots \otimes$ $V_{n}\left(z_{n}\right)$ by $\mathscr{H}_{\otimes}$. Recall from Proposition (3.1.5) that the action of the constant loops $\mathfrak{g} \subset L \mathfrak{g}$ on $\mathscr{H}_{\otimes}$ is integrable, and decomposes $\mathscr{H}_{\otimes}$ into the direct product of isotypical components, and that the same holds for the subalgebra $\mathfrak{s} \subset L \mathfrak{g}_{\mathbb{C}}$ spanned by $\left\{e_{\alpha_{\max }}(-1), h_{\alpha_{\max }}(0), e_{-\alpha_{\max }}(1)\right\}$.

Proposition 3.5.1. Assume that $(\lambda+\rho) \cdot \alpha_{\max }>h+c$. Let $\mu+\rho=\mathfrak{s} t+1(\lambda+\rho)$. (Recall from Sect. 0.3 that this equals $\left.(\lambda+\rho)+\left(h+1-\lambda \cdot \alpha_{\max }\right) \cdot \alpha_{\max }\right)$. If $(\mu+\rho)$ is a regular weight of $\mathrm{g}$, let $V^{\prime}$ be the representation of $G$ whose $\rho$ shifted highest weight $\left(\lambda^{\prime}+\rho\right)$ is in the Weyl orbit of $(\mu+\rho)$, and let $L$ be the length of the Weyl group element $w$ taking the former to the latter. Then, 
(a) If $\mu+\rho$ is singular, $H^{q}\left(\mathfrak{g}_{\mathbb{C}[z]}, \mathfrak{g}_{\mathbb{C}} ; \mathscr{H}_{\otimes} \otimes V(0)\right)=0,(\forall q)$;

(b) If $\mu+\rho$ is regular, $H^{q}\left(\mathfrak{g}_{\mathbb{C}[z]}, \mathfrak{g}_{\mathbb{C}} ; \mathscr{H}_{\otimes} \otimes V(0)\right)=H^{q-L-1}\left(\mathfrak{g}_{\mathbb{C}[z]}, \mathfrak{g}_{\mathbb{C}} ; \mathscr{H}_{\otimes} \otimes\right.$ $\left.V^{\prime}(0)\right)$.

(It is part of the statement that the LHS is 0 when $q \leqq L$.)

Remark 3.5.2. We have $\ell(V)=\ell\left(V^{\prime}\right)+L+1$. Since the length of $V^{\prime}$ is strictly less than that of $V$, repeated application of (3.5.1) results either in a singular $(\mu+\rho)$ (if $V$ was singular), or else in a final antidominant $V^{\prime}$. By applying this procedure to all the factors $V_{k}$ and using Proposition (3.4.2), we obtain a proof of Theorem 0 .

Proof of Proposition 3.5.1. The ideal $\mathfrak{g}_{+} \subset \mathfrak{g}_{\mathbb{C}[z]}$ of positive is a complement of $\mathrm{g}_{\mathbb{C}}$, and thus

$$
H^{*}\left(\mathfrak{g}_{\mathbb{C}[z]}, \mathfrak{g}_{\mathbb{C}} ; \mathscr{H}_{\otimes} \otimes V(0)\right) \cong\left[H^{*}\left(\mathfrak{g}_{+} ; \mathscr{H}_{\otimes}\right) \otimes V\right]^{G}
$$

The proposition then follows from the next two lemmas, which are simple applications of the Hochschild-Serre spectral sequence. Let $\mathfrak{N}^{+}=\mathfrak{n}_{+}(0) \tilde{\oplus} \mathfrak{g}_{+}$, where $n_{+}$ is the nilpotent subalgebra spanned by the positive ${ }^{22}$ root vectors in $\mathfrak{g}_{\mathbb{C}}$.

Lemma 3.5.4. We have

$$
\left[H^{q}\left(\mathfrak{N}^{+} ; \mathscr{H}_{\otimes}\right) \otimes \mathbb{C}_{-\lambda}\right]^{T} \cong\left[H^{q}\left(\mathfrak{g}_{+} ; \mathscr{H}_{\otimes}\right) \otimes V\right]^{G}
$$

and

$$
\begin{array}{rlrl}
{\left[H^{q+L}\left(\mathfrak{N}^{+} ; \mathscr{H}_{\otimes}\right) \otimes \mathbb{C}_{-\mu}\right]^{T}} & \cong\left[H^{q}\left(\mathfrak{g}_{+} ; \mathscr{H}_{\otimes}\right) \otimes V^{\prime}\right]^{G} \text { if }(\mu+\rho) \text { is regular } \\
& \cong 0 & \text { if }(\mu+\rho) \text { is singular } .
\end{array}
$$

Lemma 3.5.5 (Bott Reflection). If $\lambda \cdot \alpha_{\max }>h+1$, then

$$
\left[H^{q}\left(\mathfrak{N}^{+} ; \mathscr{H}_{\otimes}\right) \otimes \mathbb{C}_{-\lambda}\right]^{T} \cong\left[H^{q-1}\left(\mathfrak{N}^{+} ; \mathscr{H}_{\otimes}\right) \otimes \mathbb{C}_{-\mu}\right]^{T},
$$

where, as in Proposition 3.5.1,

$$
\mu=s_{\ell+1}(\lambda+\rho)-\rho=\lambda+\left(h+1-\lambda \cdot \alpha_{\max }\right) \cdot \alpha_{\max }
$$

Just as before, it is part of the second statement in (3.5.4) that the LHS cohomology vanishes in degree less than $L$, resp. in degree less than 1 in (3.5.5).

Proof of (3.5.4). The $E_{2}^{p, q}$ term in the Serre-Hochschild spectral sequence for the pair $\left(\mathfrak{N}^{+}, \mathfrak{g}_{+}\right)$is $H^{p}\left(\mathfrak{n}^{+} ; H^{q}\left(\mathfrak{g}_{+} ; \mathscr{H}_{\otimes}\right)\right)$. The complex is the direct product of its $T$-isotypical components; for it is the direct product of its $G$-isotypical parts, and high $T$-weights only appear in large $G$-representations. The cohomology can thus be calculated in every character of $T$ separately. However, by Kostant's version of $\mathrm{BWB}[\mathrm{K}]$, we must have, for any ${ }^{23}$ representation $E$ of $G$,

$$
\left[H^{L}\left(\mathrm{n}^{+}, E\right) \otimes \mathbb{C}_{-\mu}\right]^{T} \cong\left(E \otimes V^{\prime}\right)^{G}, \text { if } \mu \text { is regular, }
$$

\footnotetext{
22 It is the complex conjugate of the nilpotent usually considered (for instance, in BWB) in the given positive root system.

23 Any finite-dimensional representation, to be pedantic, but this certainly extends to our case, where $E$, as always, will be the direct product of isotypical components of finite-dimensional type.
} 
and

$$
\left[H^{p}\left(\mathrm{n}^{+}, E\right) \otimes \mathbb{C}_{-\mu}\right]^{T}=0 \text { for } p \neq L \text {, or when } \mu \text { is singular . }
$$

Thus in the regular case, $E_{2}^{L, q}=\left[H^{q}\left(\mathfrak{g}_{+} ; \mathscr{H}_{\otimes}\right) \otimes V^{\prime}\right]^{G}$, whereas $E_{2}^{p, q}=0$ if $p \neq L$. The spectral sequence must then collapse at $E_{2}$, and thus

$$
\left[H^{q+L}\left(\mathfrak{N}^{+} ; \mathscr{H}_{\otimes}\right) \otimes \mathbb{C}_{-\mu}\right]^{T} \cong E_{2}^{L, q}=\left[H^{q}\left(\mathfrak{g}_{+} ; \mathscr{H}_{\otimes}\right) \otimes V^{\prime}\right]^{G} .
$$

In the singular case, $E_{2}=0$. The first statement is proved in the same manner.

Proof of 3.5.5. Let $\mathfrak{u}$ be the subspace of $\mathfrak{N}^{+}$which complements the onedimensional subalgebra $\mathbb{C} \cdot e_{-\alpha_{\max }}(1)$ (in the natural basis of Fourier modes, root vectors, ...). $\mathfrak{u}$ is an ideal in $\mathfrak{N}^{+}$, and the quotient $\mathfrak{N}^{+} / \mathfrak{u}$ is isomorphic to the maximal nilpotent subalgebra $\mathfrak{m}:=\mathbb{C} \cdot e_{-\alpha_{\max }}(1)$ of $\mathfrak{s}$. The above collapsing argument, applied to the spectral sequence of the pair $\left(\mathfrak{N}^{+}, \mathfrak{u}\right)$, shows that

$$
\begin{gathered}
{\left[H^{q}\left(\mathfrak{N}^{+} ; \mathscr{H}_{\otimes}\right) \otimes \mathbb{C}_{-\lambda}\right]^{T} \cong\left[H^{1}\left(\mathrm{~m} ; H^{q-1}\left(\mathfrak{u} ; \mathscr{H}_{\otimes}\right)\right) \otimes \mathbb{C}_{-\lambda}\right]^{T},} \\
{\left[H^{q-1}\left(\mathfrak{N}^{+} ; \mathscr{H}_{\otimes}\right) \otimes \mathbb{C}_{-\mu}\right]^{T} \cong\left[H^{0}\left(\mathrm{~m} ; H^{q-1}\left(\mathfrak{u} ; \mathscr{H}_{\otimes}\right)\right) \otimes \mathbb{C}_{-\mu}\right]^{T} .}
\end{gathered}
$$

The fact that the cohomology of $m$ is confined to the indicated degrees, and the equality of the right hand-sides, are consequences of the BWB theorem for the Lie algebra $\mathfrak{s} \cong \mathfrak{s l}_{2}(\mathbb{C})$; but some further comment is necessary in order to see this. We note the fact that the restrictions to the subalgebra $\mathbb{C} \cdot h_{\alpha_{\max }}(0)$ of the actions on $\mathscr{H}_{\otimes}$ of $\mathfrak{g}_{\mathbb{C}[z]}$ and $\mathfrak{s}$ will differ by a character, because of the projective nature of the representation. Specifically, consider the twist

$$
H^{q-1}\left(\mathfrak{u} ; \mathscr{H}_{\otimes}\right) \otimes \mathbb{C}_{-h} \cdot \alpha_{\max } / 2
$$

with the obvious actions of $m$ and $T$. It is this twisted action, and not the original one, which extends compatibly to an action of $\mathfrak{s}$.

Let $\lambda^{\prime \prime}$ be a character of $T$, and set $k=\lambda^{\prime \prime} \cdot \alpha_{\max }-1$. We have defined $k$ so that we have

$$
\left(-\lambda^{\prime \prime}+k \cdot \alpha_{\max }\right) \cdot \alpha_{\max }=\lambda^{\prime \prime} \cdot \alpha_{\max }-2 .
$$

For an integrable representation $E$ of $\mathfrak{s}$, carrying a compatible action of $T$, we then have

$$
\left[H^{1}(\mathrm{~m} ; E) \otimes \mathbb{C}_{-\lambda^{\prime \prime}}\right]^{T} \cong\left[H^{0}(\mathrm{~m} ; E) \otimes \mathbb{C}_{\lambda^{\prime \prime}+k} \cdot \alpha_{\max }\right]^{T} .
$$

(We are forced to write this well-known identity in a rather unnatural way so as not to interfere with the action of the subalgebra of $t$ orthogonal to $h_{\alpha_{\max }}$.) Let

$$
\lambda^{\prime \prime}=\lambda-\frac{h}{2} \cdot \alpha_{\max }, \quad \mu^{\prime \prime}=\mu-\frac{h}{2} \cdot \alpha_{\max } .
$$

Taking (3.5.7) as our space $E$, we have

$$
\left[H^{*}(\mathfrak{m} ; E) \otimes \mathbb{C}_{-\lambda^{\prime \prime}}\right]^{T} \cong\left[H^{*}\left(\mathfrak{m} ; H^{q-1}\left(\mathfrak{u} ; \mathscr{H}_{\otimes}\right)\right) \otimes \mathbb{C}_{-\lambda}\right]^{T}
$$

and correspondingly for $\mu$. But note that

$$
\begin{aligned}
-\lambda^{\prime \prime}+k \cdot \alpha_{\max } & =-\lambda+\frac{h}{2} \cdot \alpha_{\max }+\left(\lambda \cdot \alpha_{\max }-h-1\right) \cdot \alpha_{\max } \\
& =-\mu+\frac{h}{2} \cdot \alpha_{\max }=-\mu^{\prime \prime}
\end{aligned}
$$


and the Reflection Lemma follows now from (3.5.9) and (3.5.10). Note also that the condition $\lambda \cdot \alpha_{\max }>h+1$ corresponds to $\lambda^{\prime \prime} \cdot \alpha_{\max }>1$, which ensures that the cohomology (3.5.10) is confined to degree 1. (Correspondingly, the $\mu$-cohomology will live in degree 0. )

3.6. Application: The Fusion Rules. Recall some definitions from Sect. 1.5: $R(G)$ is the representation ring of $G$, while $A_{h}$ is the free abelian group based on the set $B_{h}$ of irreducible positive energy representations of the loop group $L G$ at level $h$. We shall take the representations in $B_{h}$ to consist of finite-energy vectors; their linear duals will then be formally complete representations, as in Sect. 3.1. Also recall Bott's holomorphic induction map (1.5.4) $i_{h}: R(G) \rightarrow A_{h}$. As one might expect, it has an elegant expression in Lie algebra cohomology terms.

Proposition 3.6.1. For a general, not necessarily irreducible representation $E$ of $G$, we have

$$
i_{h}(E)=\bigoplus_{\mathscr{H} \in B_{h}} \mathscr{H} \cdot \chi\left(\mathfrak{g}_{\mathbb{C}[z]}, \mathfrak{g}_{\mathbb{C}} ; \mathscr{H}^{t} \otimes E(0)\right)
$$

Proof. This is a consequence of part (a) of Theorem 0 .

Remark 3.6.2. Given the level $h$, the Kac character formula [P-S, Sect. 14] assigns to every weight $\lambda$ of $\mathfrak{g}$ a formal character $\chi_{K}(\lambda)$ in $R(G)[[u]]$ - a representation of $G \times S^{1}$ with finite-dimensional $S^{1}$-eigenspaces. ( $S^{1}$ represents the energy action.) If $\lambda_{E}$ is the highest weight of $E$, one can show that the Kac character $\chi_{K}\left(\lambda_{E}\right)$ is the character of the representation $i_{h}(E)$ in (3.6.1).

We shall use the following definition of fusion [T-Y]. Pick $n+1$ points $z_{0}, z_{1}, \ldots, z_{n} \in \mathbb{P}^{1}$. Let $K$ denote the ring of rotational functions on $\mathbb{P}^{1}$ whose only poles are at the points $\left\{z_{k}\right\}$. The group $G_{k}$ is naturally contained in the product $G\left(\mathscr{M}_{z_{0}}\right) \times \cdots \times G\left(\mathscr{M}_{z_{n}}\right)$. In keeping with the previous sections, we shall take $z_{0}=\infty$. This is not a restriction, because of the $S L_{2}(\mathbb{C})$-invariance of the construction below.

Given PERs $\mathscr{H}, \mathscr{H}_{1}, \ldots, \mathscr{H}_{n}$ of $L G$, set

$$
\hat{\mathscr{H}}_{0}(\infty):=\mathscr{H}^{t}, \quad \hat{\mathscr{H}}_{k}\left(z_{k}\right):=\hat{\mathscr{H}}_{V_{k}}\left(z_{k}\right) \text { for } k \geqq 1,
$$

where $\mathscr{H}^{t}$ is the formally completed dual of $\mathscr{H}$ and $V_{k}$ is the space of ground states of $\mathscr{H}_{k}$. (Thus $\hat{\mathscr{H}}_{0}$ is based on the dual of the space of ground states of $\mathscr{H}_{\text {.) }}$ The energy co-filtrations on the spaces $\hat{\mathscr{H}}_{k}\left(z_{k}\right)$ and $\mathscr{H}^{t}$ lead to a co-filtration on $\hat{\mathscr{H}}_{0}(\infty) \otimes \cdots \otimes \hat{\mathscr{H}}_{n}\left(z_{n}\right)$. Its formal completion $\hat{\mathscr{H}}_{0}(\infty) \hat{\otimes} \cdots \hat{\otimes}_{\hat{H}_{n}}\left(z_{n}\right)$ thereunder is aprojective representation of $G\left(\mathscr{M}_{z_{0}}\right) \times \cdots \times G\left(\mathscr{M}_{z_{n}}\right)$, which restricts in a unique way to a genuine representation of the subgroup $G_{K}$. ( $G_{K}$ is connected, and the projective cocycle on the associated Lie algebra $\mathfrak{g}_{K}$ is trivial; in addition $\mathfrak{g}_{K}$ admits no non-trivial characters.) The spaces of invariants (cf. 1.5.1)

$$
M\left(\mathscr{H} \mid \mathscr{H}_{1}, \ldots, \mathscr{H}_{n}\right):=\left(\hat{\mathscr{H}}_{0}(\infty) \hat{\otimes} \hat{\mathscr{H}}_{1}\left(z_{1}\right) \hat{\otimes} \cdots \hat{\otimes} \hat{\mathscr{H}}_{n}\left(z_{n}\right)\right)^{G_{K}}
$$

are therefore well-defined, and we are interested in their dimensions, which we encode in the $n$-ary fusion product $\circledast(\ldots, \ldots, \ldots)$, an $n$-linear operation from $A_{h} \times$ $\cdots \times A_{h}$ to $A_{h}$ :

Definition 3.6.4. $\circledast\left(\mathscr{H}_{1}, \ldots, \mathscr{H}_{n}\right):=\bigoplus_{\mathscr{H} \in B_{h}} \mathscr{H} \cdot \operatorname{dim} M\left(\mathscr{H} \mid \mathscr{H}_{1}, \ldots, \mathscr{H}_{n}\right)$. 
For the last definition to make sense, the dimensions of the $M(\mid)$ 's must be finite and independent of the choice of points $z_{k}$. This follows from the following observation of G. Segal's, which relates the fusion coefficients to the Lie algebra invariants discussed earlier.

Lemma 3.6.5. (G. Segal). One has a natural isomorphism

$$
\left(\mathscr{H}^{t} \hat{\otimes} \hat{\mathscr{H}}_{V_{1}}\left(z_{1}\right) \hat{\otimes} \cdots \hat{\otimes} \hat{\mathscr{H}}_{V_{n}}\left(z_{n}\right)\right)^{G(k)} \cong\left(\mathscr{H}^{t} \otimes V_{1}\left(z_{1}\right) \otimes \cdots \otimes V_{n}\left(z_{n}\right)\right)^{G(\mathbb{C}[z])} .
$$

Remark. Recalling that $\hat{\mathscr{H}}_{k}\left(z_{k}\right)$ is a submodule of $\Gamma_{V_{k}}\left(z_{k}\right)=\operatorname{Hom}_{\mathfrak{g}\left(c_{z_{k}}\right)}$ $\left(U_{h}\left(g\left(\mathscr{M}_{z_{k}}\right)\right) ; V_{k}\left(z_{k}\right)\right)$, the natural map from left to right is the restriction to $1 \otimes \cdots \otimes 1 \in U_{h}\left(\mathfrak{g}\left(\mathscr{M}_{z_{1}}\right)\right) \otimes \cdots \otimes U_{h}\left(\mathfrak{g}\left(\mathscr{M}_{z_{n}}\right)\right)$.

Corollary 3.6.6. With $\mathscr{H}^{t}$ denoting the formally completed dual of $\mathscr{H}$, we have

$$
\operatorname{dim} M\left(\mathscr{H} \mid \mathscr{H}_{1}, \ldots, \mathscr{H}_{n}\right)=h^{0}\left(\mathfrak{g}_{\mathbb{C}[z]} \mathfrak{g}_{\mathbb{C}} ; \mathscr{H}^{t} \otimes \vec{V}(\vec{z})\right) .
$$

Remark. Corollary 3.6.6 does contain the statement that $G_{\mathbb{C}[z]}$-invariants and $\mathfrak{g}_{\mathbb{C}[z]^{-}}$ invariants are the same. This follows from the connectedness of $G$ (cf. [F]).

Proof of (3.6.5). The lemma admits a Lie algebra proof, using the complete reducibility of $\mathscr{H}$, but the following geometric argument is more appealing. Let us use the realization of $\hat{\mathscr{H}}_{k}\left(z_{k}\right)$ as the space of holomorphic sections of the vector bundle $\mathbf{V}_{k}=G\left(\mathscr{M}_{z_{k}}\right) \times_{G\left(\mathcal{C}_{z_{k}}\right)} V_{k}\left(z_{k}\right)$, twisted by $\mathscr{L}^{\otimes h}$, as in Prop. 3.1.5, part ii. The left-hand side of (3.6.5) is the space of left $G(K)$-invariant sections over $\prod_{k=1}^{n} G\left(\mathscr{M}_{z_{k}}\right) / G\left(\mathcal{O}_{z_{h}}\right)$ of the vector bundle

$$
\mathscr{H}^{t} \otimes\left(\mathbf{V}_{1} \otimes \mathscr{L}^{\otimes h}\right) \otimes \cdots \otimes\left(\mathbf{V}_{n} \otimes \mathscr{L}^{\otimes h}\right) .
$$

( $\mathscr{H}^{t}$ abusively denotes the trivial vector bundle with fibre $\mathscr{H}^{t}$.) As we shall see momentarily, we have

$$
\prod_{k=1}^{n} G\left(\mathscr{M}_{z_{k}}\right) / G\left(\mathcal{O}_{z_{k}}\right) \cong G(K) / G(\mathbb{C}[z])
$$

as left $G(K)$-spaces, with the obvious action of $G(K)$ on the spaces $G\left(\mathscr{M}_{z_{h}}\right) / G\left(\mathcal{O}_{z_{k}}\right)$. Equation (3.6.8) then identifies the left $G(K)$-invariant sections of the vector bundle (3.6.7) with the $G(\mathbb{C}[z])$-invariants in its fibre at the identity coset. But the latter is precisely

$$
\left(\mathscr{H}^{t} \otimes V_{1}\left(z_{1}\right) \otimes \cdots \otimes V_{n}\left(z_{n}\right)\right)^{G_{\mathbb{C}[z]}} .
$$

To see (3.6.8), note first that the obvious map goes from right to left. Conversely, a point $\left(g_{1}, \ldots, g_{n}\right)$ in the left-hand space can be viewed as a set of holo-

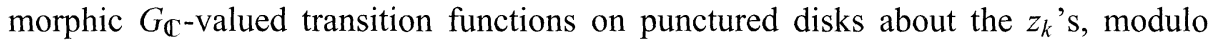
local holomorphic gauge transformations. In other words, it determines a holomorphic (thus algebraic) $G_{\mathbb{C}}$-bundle over $\mathbb{P}^{1}$, together with a meromorphic section $\mathbf{1}$, with poles only at the $z_{k}$ 's. This bundle, when restricted to $\mathbb{C}=\mathbb{P}^{1} \backslash\{0\}$, must admit an algebraic section $\sigma$, unique up to right multiplication by $G_{\mathbb{C}[z]}$. We then have $\sigma=\mathbf{1} \cdot \mu$, with $\mu \in G_{K}$, and $\mu$ gives the desired representative of $\left(g_{1}, \ldots, g_{n}\right)$ on the right-hand side.

Theorem 1 ("The Fusion Rules"). Holomorphic induction is a ring homomorphism. More precisely, 
(a) the fusion operation $\circledast(\ldots, \ldots, \ldots)$ comes from a commutative and associative multiplication $\circledast$,

(b) $i_{h}:(R(G), \otimes) \rightarrow\left(A_{h}, \circledast\right)$ is a surjective ring homomorphism. For $V_{k} \in$ $R(G), k=1, \ldots, n$, we thus have

$$
\circledast\left(i_{h}\left(V_{1}\right), \ldots, i_{h}\left(V_{n}\right)\right)=i_{h}\left(V_{1} \otimes \cdots \otimes V_{n}\right) .
$$

Remark. It is a consequence of our statement that the kernel of the map $i_{h}$ is an ideal in $R(G)$. This could also be proved directly from the Weyl-Kac character formula.

$$
\begin{array}{rlr}
\text { Proof. } \circledast\left(i_{h}\left(V_{1}\right), \ldots i_{h}\left(V_{n}\right)\right) & =\bigoplus_{\mathscr{H} \in B_{h}} \mathscr{H} \cdot \operatorname{dim} M\left(\mathscr{H} \mid \mathscr{H}_{1}, \ldots, \mathscr{H}_{n}\right) \text { (by definition) } \\
& =\bigoplus_{\mathscr{H} \in B_{h}} \mathscr{H} \cdot h^{0}\left(\mathscr{H}^{t} \otimes \vec{V}(\vec{z})\right) & \text { (Segal's Lemma) } \\
& =\bigoplus_{\mathscr{H} \in B_{h}} \mathscr{H} \cdot \chi\left(\mathscr{H}^{t} \otimes \vec{V}(\vec{z})\right) & \text { (Vanishing theorem) } \\
& =\bigoplus_{\mathscr{H} \in B_{h}} \mathscr{H} \cdot \chi\left(\mathscr{H}^{t} \otimes \vec{V}(\overrightarrow{0})\right) & \text { (Rigidity of } \chi) \\
& =i_{h}\left(V_{1} \otimes \cdots \otimes V_{n}\right) . & \text { (Proposition 3.6.1) }
\end{array}
$$

\section{Appendix A. Lie Algebra Cohomology and the Hochschild-Serre Spectral Sequence}

In this section we recall a number of basic facts about Lie algebra cohomology used in this paper.

1. Lie Algebra Cohomology. Let $\mathfrak{g}$ be a finite or infinite-dimensional Lie algebra over a ground field $k$, which we take to be $\mathbb{R}$ or $\mathbb{C}$. The category $\mathscr{C}(\mathfrak{g})$ of all representations of $\mathfrak{g}$ over $k$ is an abelian category with enough injectives. (It is the same as the category of $U(\mathfrak{g})$-modules.) The fixed point functor, taking a representation $V$ of $\mathfrak{g}$ to the space $V^{\mathfrak{g}}$ of $\mathfrak{g}$-invariant vectors, is left exact, and its right derived functors, applied to $V$, are the Lie algebra cohomology groups $H^{*}(\mathfrak{g} ; V)$.

The trivial $\mathfrak{g}$-module $k$ has a canonical free resolution in $U(\mathfrak{g})$ :

$$
0 \leftarrow k \leftarrow U(\mathfrak{g}) \leftarrow U(\mathfrak{g}) \otimes \Lambda^{1}(\mathfrak{g}) \leftarrow U(\mathfrak{g}) \otimes \Lambda^{2}(\mathfrak{g}) \leftarrow U(\mathfrak{g}) \otimes \Lambda^{3}(\mathfrak{g}) \leftarrow \cdots .
$$

If $\mathfrak{g}$ is the Lie algebra of a Lie group $G$, the dual resolution

$$
\begin{aligned}
0 \rightarrow k \rightarrow \operatorname{Hom}(U(\mathfrak{g}) ; k) & \rightarrow \operatorname{Hom}\left(U(\mathfrak{g}) \otimes \Lambda^{1}(\mathfrak{g}) ; k\right) \\
& \rightarrow \operatorname{Hom}\left(U(\mathfrak{g}) \otimes \Lambda^{2}(\mathfrak{g}) ; k\right) \rightarrow \cdots
\end{aligned}
$$

is the De Rham complex of forms with formal power series coefficients at the identity of $G$. The resolution A.1.1 leads to a $\mathscr{C}(\mathfrak{g})$-injective resolution for any $V \in \mathscr{C}(\mathfrak{g})$,

$$
\begin{aligned}
0 \rightarrow V \rightarrow \operatorname{Hom}_{k}(U(\mathfrak{g}) ; V) & \rightarrow \operatorname{Hom}_{k}\left(U(\mathfrak{g}) \otimes \Lambda^{1}(\mathfrak{g}) ; V\right) \\
& \rightarrow \operatorname{Hom}_{k}\left(U(\mathfrak{g}) \otimes \Lambda^{2}(\mathfrak{g}) ; V\right) \rightarrow \cdots .
\end{aligned}
$$


(The $\mathfrak{g}$-module structure on $\operatorname{Hom}_{k}\left(U(\mathfrak{g}) \otimes \Lambda^{*}(\mathfrak{g}) ; V\right)$ comes from left multiplication on $U(\mathfrak{g})$ and from the natural action on $V$.)

Applying the functor of $\mathfrak{g}$-invariants to (A.1.2) gives a resolution of cohomology known as the standard complex of Lie algebra cohomology:

$$
\begin{aligned}
0 \longrightarrow V \stackrel{\partial}{\longrightarrow} \operatorname{Hom}_{k}\left(\Lambda^{1}(\mathfrak{g}) ; V\right) & \stackrel{\partial}{\longrightarrow} \operatorname{Hom}_{k}\left(\Lambda^{2}(\mathfrak{g}) ; V\right) \\
& \stackrel{\partial}{\longrightarrow} \operatorname{Hom}_{k}\left(\Lambda^{3}(\mathfrak{g}) ; V\right) \stackrel{\partial}{\longrightarrow} \cdots .
\end{aligned}
$$

In a basis $\left\{\gamma_{k}\right\}$ of $\mathfrak{g}$, the coboundary operator $\partial$ is explicitly given by

$$
\partial=\sum_{k}\left(R\left(\gamma_{k}\right) \otimes \varepsilon\left(\gamma^{k}\right)+\frac{1}{2} \varepsilon\left(\gamma^{k}\right) \cdot \mathrm{ad}_{\gamma_{k}}^{t}\right),
$$

where $\left\{\gamma^{k}\right\}$ is the dual basis of $\mathfrak{g}^{t}, \varepsilon$ denotes exterior multiplication on $\Lambda^{*}(\mathfrak{g})$ and $R$ is the action of $\mathfrak{g}$ on $V$.

There is a natural action of $\mathfrak{g}$ on the spaces $\operatorname{Hom}\left(\Lambda^{*}(\mathfrak{g}) ; V\right)$, obtained from the ad-action on $g$ and the natural action on $V$. This action commutes with the $\partial$-operator. However, the induced action on the cohomology spaces is trivial: the action of $\gamma \in \mathfrak{g}$ can also be expressed as the (super)commutator of $\partial$ with the interior multiplication $\imath(\gamma)$.

2. Relative Lie Algebra Cohomology. Let now $\mathfrak{h}$ be a subalgebra of $\mathfrak{g}$. Suppose that $\mathfrak{h}$ is the Lie algebra of a connected Lie group $H$, which we assume to be reductive. We also assume that $\mathfrak{g}$ splits into finite-dimensional integrable $\mathfrak{h}$-modules under the restricted ad-action. The category $\mathscr{C}(\mathfrak{g})$ contains the full subcategory $\mathscr{C}(\mathfrak{g}, H)$ of modules which split as a direct product ${ }^{24}$ of their $\mathfrak{h}$-isotypical components, all of which are of finite-dimensional $H$-type. $\mathscr{C}(\mathfrak{g}, H)$ is also an abelian category with enough injectives. The functor of $\mathfrak{g}$-invariants is again left exact, and its right derived functors are the relative Lie algebra cohomology groups $H^{*}(\mathfrak{g}, \mathfrak{h} ; V)$ of the pair $(\mathfrak{g}, \mathfrak{h})$. Every $V \in \mathscr{C}(\mathfrak{g}, H)$ has a canonical injective resolution

$$
\begin{aligned}
0 \rightarrow V \rightarrow \operatorname{Hom}_{\mathfrak{h}}(U(\mathfrak{g}) ; V) & \rightarrow \operatorname{Hom}_{\mathfrak{h}}\left(U(\mathfrak{g}) \otimes \Lambda^{1}(\mathfrak{g} / \mathfrak{h}) ; V\right) \\
& \rightarrow \operatorname{Hom}_{\mathfrak{h}}\left(U(\mathfrak{g}) \otimes \Lambda^{2}(\mathfrak{g} / \mathfrak{h}) ; V\right) \rightarrow \cdots,
\end{aligned}
$$

which is a subcomplex of (A.1.2). "Hom right multiplication on $U(\mathfrak{g})$, by "ad" on $\mathfrak{g} / \mathfrak{h}$ ) and by restriction of the $\mathfrak{g}$-action on $V$.

Again, applying the left exact functor of $g$-invariants leads to a standard complex

$$
\begin{aligned}
0 \rightarrow V \stackrel{\partial}{\longrightarrow} \operatorname{Hom}_{\mathfrak{h}}\left(\Lambda^{1}(\mathfrak{g} / \mathfrak{h}) ; V\right) \stackrel{\partial}{\longrightarrow} \operatorname{Hom}_{\mathfrak{l}}\left(\Lambda^{2}(\mathfrak{g} / \mathfrak{h}) ; V\right) \\
\stackrel{\partial}{\longrightarrow} \operatorname{Hom}_{\mathfrak{h}}\left(\Lambda^{3}(\mathfrak{g} / \mathfrak{h}) ; V\right) \stackrel{\partial}{\longrightarrow} \cdots .
\end{aligned}
$$

It is a subcomplex of (A.1.3), and its cohomology is the relative Lie algebra cohomology $H^{*}(\mathfrak{g}, \mathfrak{h} ; V)$.

3. The Case when $\mathfrak{h}$ is Complemented. Suppose that $\mathfrak{h} \subset \mathfrak{g}$ has a complement $\mathfrak{p}$ which is an ideal in $\mathfrak{g} ; \mathfrak{g}$ then splits as a semidirect sum $\mathfrak{g}=\mathfrak{h} \tilde{\oplus} \mathfrak{p}$ of $\mathfrak{p}$ by $\mathfrak{h}$. For a $\mathscr{C}(\mathfrak{g}, H)$-module $V$, there is a natural isomorphism

$$
H^{*}(\mathfrak{g}, \mathfrak{h} ; V) \cong\left(H^{*}(\mathfrak{p} ; V)\right)^{H}
$$

\footnotetext{
24 It is more common to require a direct sum, rather than product, decomposition, but this suits our examples better. The important property of $\mathscr{C}(\mathfrak{g}, H)$ is that any exact sequence in it is split as a sequence of $\mathfrak{h}$-modules.
} 
of the space $H^{*}(\mathfrak{g}, \mathfrak{h} ; V)$ with the space of $H$-invariant vectors in $H^{*}(\mathfrak{p} ; V)$. This is because the standard relative complex (A.2.2) for the pair $(\mathfrak{g}, \mathfrak{h})$ is the $H$-invariant part of the standard complex (A.1.3) for $\mathfrak{p}$, and the splitting into $H$-isotypical factors ensures that the cohomology can be calculated in every isotypical component separately.

4. Hochschild-Serre Spectral Sequence. Let now $\mathfrak{p}$ be an ideal in $\mathfrak{g}$. Given a representation $V$ of $\mathfrak{g}$, there exists a spectral sequence converging to $H^{*}(\mathfrak{g} ; V)$, whose $E_{2}$ term is $H^{*}\left(\mathfrak{g} / \mathfrak{p} ; H^{*}(\mathfrak{p} ; V)\right)$. (g acts on $V$, and acts compatibly on $\mathfrak{p}$ by infinitesimal automorphisms; thus it acts on $H^{*}(\mathfrak{p} ; V)$, and the subalgebra $\mathfrak{p}$ acts trivially.) The existence of such a spectral sequence springs from the fact that the functor of $\mathfrak{g}$-invariants can be expressed as the composition of $\mathfrak{p}$-invariants and $\mathfrak{g} / \mathfrak{p}$-invariants. We can be slightly more specific. The $\mathfrak{g}$-invariant projection $\mathfrak{g} \rightarrow \mathfrak{g} / \mathfrak{p}$ leads to a dual inclusion $\mathfrak{g}^{t} \supset(\mathfrak{g} / \mathfrak{p})^{t}$, which can be thought of as a $\mathfrak{g}$-invariant twostep filtration of $\mathfrak{g}^{t}$. This naturally leads to a filtration of $\operatorname{Hom}\left(\Lambda^{*}(\mathfrak{g}) ; V\right)$, whose associated graded module is

$$
\operatorname{Hom}\left(\Lambda^{*}(\mathfrak{g} / \mathfrak{p}) \otimes \Lambda^{*}(\mathfrak{p}) ; V\right) \cong \operatorname{Hom}\left(\Lambda^{*}(\mathfrak{g} / \mathfrak{p}) ; \operatorname{Hom}\left(\Lambda^{*}(\mathfrak{p}) ; V\right)\right) .
$$

This filtration has finite length if $\mathfrak{p}$ has finite codimension in $\mathfrak{g}$.

From this filtration of the standard cohomology complex, one proceeds in the usual manner to set up a spectral converging to $H^{*}(\mathrm{~g} ; V)$, the Hochschild-Serre spectral sequence. A direct verification shows that the first differential $\delta_{1}$ of the coboundary operator $\partial_{\mathfrak{g}}$ can be identified with the coboundary operator $\partial_{\mathfrak{p}}$ of $\mathfrak{p}$ acting on $\operatorname{Hom}\left(\Lambda^{*}(\mathfrak{p}) ; V\right)$ (the $\mathfrak{p}$-action on $\Lambda^{*}(\mathfrak{g} / \mathfrak{p})$ is trivial), while the second differential $\delta_{2}$ agrees with $\partial_{\mathfrak{g} / \mathfrak{p}}$, acting on $\operatorname{Hom}\left(\Lambda^{*}(\mathfrak{g} / \mathfrak{p}) ; H^{*}\left(\Lambda^{*}(\mathfrak{p}) ; V\right)\right)$. Thus $E_{2}^{p, q}=H^{p}\left(\mathfrak{g} / \mathfrak{p} ; H^{q}(\mathfrak{p} ; V)\right)$, and in a given cohomology degree $d$, the sequence collapses after at most $d$ steps. Its stable limit is $\operatorname{Gr}\left(H^{*}(\mathfrak{g} ; V)\right)$, the graded vector space associated to the filtration induced on the cohomology groups $H^{*}(\mathfrak{g} ; V)$.

\section{Appendix B. Two Useful Identities}

B.1. Nakano's Identity. There are to my knowledge two proofs of Nakano's identity (2.4.7),

$$
\square=\bar{\square}-(h+2 c) \cdot q-T_{\vec{V}(\vec{z})}^{A} \text { on } \mathscr{C}^{0, q} .
$$

They are both based on classical finite-dimensional arguments, and use the Kähler differential calculus on $X=L G / G$. The extraneous ingredient, in one proof, is the "anomalous" behaviour (B.1.11) of the differential $d$ in the semi-infinite cohomology of $L g_{\mathbb{C}}$. This proof is easily packaged as a Lie algebra argument and I shall present it below. The other proof is a direct differential-geometric calculation of the Laplacians on $X$. I do not have a good Lie algebra cohomology translation for it, but I should mention that the calculations involve the Ricci curvature of the Kähler metric on $X$. The latter was determined by Freed [Fr] to be $2 c \cdot \omega$. This expresses the fact that the canonical bundle of $X$ is $\mathscr{L}^{\otimes(-2 c)}$, and is the geometric counterpart of the extra cocycle $2 c \cdot \omega$ appearing in the square of the semi-infinite differential (B.1.10).

Proof of Nakano's Identity. The proof is broken down in the simple steps (i)-(ix); most noteworthy are the Kähler identities (B.1.6). Let

$$
\mathscr{C}^{-p, q}(\hat{\mathscr{H}} \otimes \vec{V}(\vec{z})):=\operatorname{Hom}_{\mathfrak{g}}\left[\Lambda^{q}\left(\mathfrak{g}_{+}\right) ; \hat{\mathscr{H}} \otimes \vec{V}(\vec{z}) \otimes \Lambda^{p}\left(\overline{\mathfrak{g}_{+}}\right)\right],
$$


where, as before, $\mathfrak{g}_{+}=\bigoplus_{k>0} \mathfrak{g}_{\mathbb{C}}(k)$. The spaces $\operatorname{Hom}\left[\Lambda^{q}\left(\mathfrak{g}_{+}\right) ; \Lambda^{p}\left(\overline{\mathfrak{g}_{+}}\right)\right]$should be viewed as exterior powers of the dual of $L \mathfrak{g}_{\mathbb{C}} / \mathfrak{g}_{\mathbb{C}}$ near the top exterior power $\Lambda^{\text {top }}\left(\left(\mathfrak{g}_{+}\right)^{t}\right)$.

As pre-Hilbert spaces. $\left(\mathfrak{g}_{+}\right)^{t}$ and $\overline{\mathfrak{g}_{+}}$are isomorphic, by means of the Kähler metric (2.1.1). Denote this isomorphism, and its multilinear extension to the exterior algebras $\Lambda^{*}\left(\left(\mathfrak{g}_{+}\right)^{t}\right)$ and $\Lambda^{*}\left(\overline{\mathfrak{g}_{+}}\right)$, by “*” ("Hodge star"); note that $*^{2}=1$. Also let $\mathscr{C}_{\mathrm{Hilb}}^{-p, q}$ be the space of Hilbert-Schmidt operators in B.1.1 (cf. 2.1.2). All the operators considered in this section are at least densely defined on $\mathscr{C}_{\mathrm{Hilb}}^{-p, q}$.

The vector space isomorphisms

$$
\text { (a) } \overline{\mathfrak{g}_{+}} \cong L \mathfrak{g}_{\mathbb{C}} / \mathfrak{g}_{\mathbb{C}[z]}, \quad \text { (b) } \quad \mathfrak{g}_{+} \cong L \mathfrak{g}_{\mathbb{C}} / \mathfrak{g}_{\mathbb{C}\left[z^{-1}\right]}
$$

give an action of $\mathfrak{g}_{+}$on $\left(\overline{\mathfrak{g}_{+}}\right)$and one of $\left(\overline{\mathfrak{g}_{+}}\right)$on $\mathfrak{g}_{+}$. We already have the ad-actions of these Lie algebras on themselves; we shall use the notation

$$
\begin{aligned}
& \operatorname{ad}_{*} \text {, to denote the actions of } \mathfrak{g}_{+} \text {and }\left(\overline{\mathfrak{g}_{+}}\right) \text {on } \operatorname{Hom}\left(\ldots ; \Lambda^{*}\left(\overline{\mathfrak{g}_{+}}\right)\right) \\
& \operatorname{ad}_{*}^{t} \text {, to denote the actions of } \mathfrak{g}_{+} \text {and }\left(\overline{\mathfrak{g}_{+}}\right) \text {on } \operatorname{Hom}\left(\Lambda^{*}\left(\overline{\mathfrak{g}_{+}}\right) ; \ldots\right)
\end{aligned}
$$

Also, define

$$
\varepsilon^{a}(n):=\text { interior }^{25} \text { multiplication by } \xi^{a}(n) \in\left(\overline{\mathfrak{g}_{+}}\right)^{t} \text { on } \mathscr{C}^{*, *}
$$

and recall that $\theta_{\vec{z}}$ represents the actions of $\mathfrak{g}_{+}$and $\overline{\mathfrak{g}_{+}}$on $\vec{V}(\vec{z})$. These actions allow us to regard the spaces $\mathscr{C}_{\text {Hilb }}^{-p, q}$ as either

(a) the (Hilbert space completed) $\mathfrak{g}$-invariant part of the standard complex of Lie algebra cohomology of $\mathfrak{g}_{+}$, with coefficients in $\hat{\mathscr{H}} \otimes \vec{V}(\vec{z}) \otimes \Lambda^{p}\left(\overline{\mathfrak{g}_{+}}\right)$, with the coboundary operator given by

$$
\bar{\partial}_{\vec{z}}=\sum_{m=1}^{\infty} \varepsilon^{a}(-m) \cdot\left(R\left(\xi_{a}(m)+\operatorname{ad}_{\xi_{a}(m)}+\frac{1}{2} \operatorname{ad}_{\xi_{a}(m)}^{t}\right)+\sum_{m=1}^{\infty} \varepsilon^{a}(-m) \cdot \theta_{\vec{z}}\left(\xi_{a}(m)\right),\right.
$$

(b) the (Hilbert space completed) $\mathfrak{g}$-invariant part of the standard complex of Lie algebra homology of $\overline{\mathfrak{g}_{+}}$, with coefficients in $\operatorname{Hom}\left[\Lambda^{q}\left(\mathfrak{g}_{+}\right) ; \hat{\mathscr{H}} \otimes \vec{V}(\vec{z})\right]$; the boundary operator is given by

$$
D_{\vec{z}}=\sum_{m=1}^{\infty}\left(R\left(\xi_{a}(-m)+\operatorname{ad}_{\xi_{a}(-m)}^{t}+\frac{1}{2} \operatorname{ad}_{\xi_{a}(-m)}\right) \cdot \varepsilon^{a}(m)+\sum_{m=1}^{\infty} \theta_{\vec{z}}\left(\xi_{a}(-m)\right) \cdot \varepsilon^{a}(m) .\right.
$$

Remark B.1.4. We have $\mathfrak{g}_{+} \cong \mathfrak{g}_{\mathbb{C}[z]} / \mathfrak{g}_{\mathbb{C}}$, hence a natural inclusion

$$
\begin{aligned}
\mathscr{C}^{*, *} & =\operatorname{Hom}_{\mathfrak{g}}\left(\Lambda^{q}\left(\mathfrak{g}_{+}\right) ; \hat{\mathscr{H}} \otimes \vec{V}(\vec{z}) \otimes \Lambda^{p}\left(\overline{\mathfrak{g}_{+}}\right)\right) \\
& \subset \operatorname{Hom}\left(\Lambda^{q}\left(\mathfrak{g}_{\mathbb{C}[z]}\right) ; \hat{\mathscr{H}} \otimes \Lambda^{p}\left(\overline{\mathfrak{g}_{+}}\right) \otimes \vec{V}(\vec{z})\right) .
\end{aligned}
$$

25 It is exterior multiplication if we think of elements of (B.1.1) as semi-infinite forms. 
In (a), $\mathscr{C}^{-p, *}$ may also be conceived as the complex of relative Lie algebra cohomology of the pair $\left(\mathfrak{g}_{\mathbb{C}[z]}, \mathfrak{g}_{\mathbb{C}}\right)$; the coboundary operator $\bar{\partial}_{\vec{z}}$ of (B.1.3.a) is then obtained as the restriction of an operator $\bar{\partial}_{\vec{z}}^{\#}$ on $\operatorname{Hom}\left(\Lambda^{*}\left(\mathfrak{g}_{\mathbb{C}[z]}\right) ; \ldots\right) . \vec{\partial}_{\vec{z}}^{\#}$ is given by the same formula (B.1.3.a), except that the summation is extended to $m=0$, and $\mathrm{ad}_{*}^{t}$ is the co-adjoint action of $\mathfrak{g}_{\mathbb{C}[z]}$ on $\left(\mathfrak{g}_{\mathbb{C}[z]}\right)^{t}$. The operator $D_{\vec{z}}$, on the other hand, extends naturally to $\operatorname{Hom}\left(\Lambda^{*}\left(\mathfrak{g}_{\mathbb{C}[z]}\right) ; \ldots\right)$ - with the summation still restricted to $m>0$-provided $\mathrm{ad}_{*}^{t}$, in (B.1.2), is modified to denote the action of $\overline{\mathfrak{g}_{+}}$on $\mathfrak{g}_{\mathbb{C}[z]} \cong L \mathfrak{g}_{\mathbb{C}} / \overline{\mathfrak{g}_{+}}$. We shall use these extended operators below.

As in Sect. 2.3, write $\bar{\partial}_{\vec{z}}=\bar{\partial}_{0}+\bar{\delta}_{\vec{z}}, \bar{\partial}_{\vec{z}}^{\#}=\bar{\partial}_{\vec{z}}^{\#}+\bar{\delta}_{\vec{z}}^{\#}$ and $D_{\vec{z}}=D_{0}+\delta_{\vec{z}}$, where the $\theta$-part of the differentials is contained in the $\delta^{\prime}$ s. Observe that $\bar{\partial}_{0}, \bar{\partial}_{0}^{\#}$ and $D_{0}$ preserve the total energy grading on $\mathscr{C}^{*, *}$, while the energy modes of $\bar{\delta}_{\vec{z}}$ and $\delta_{\vec{z}}$ have exponential decay. This can be used to prove that the compositions and commutators below are densely defined, and justifies the formal identities we shall derive.

We make note of the following easily verified facts:

(i) $\left(\mathscr{C}_{\mathrm{H} \text { lb }}^{0, *} ; \bar{\partial}_{\vec{z}}\right)=\left(\mathscr{C}_{\mathrm{Hilb}}^{*} ; \bar{\partial}_{\vec{z}}\right)$, the complex discussed in Chap. 2.

(ii) $D_{\vec{z}}^{*}=* \bar{\partial}_{\vec{z}} *$, and $\bar{\partial}_{\vec{z}}^{*}=* D_{\vec{z}} *$.

(iii) Let $\square:=\left[D_{\vec{z}}, D_{\vec{z}}^{*}\right]=D_{\vec{z}} D_{\vec{z}}^{*}+D_{\vec{z}}^{*} D_{\vec{z}}$. Then, $\square$, restricted to $\mathscr{C}^{0, *}$, is the operator $\square$ defined in (2.4.6).

Consider now the standard Kähler operators on $\mathscr{C}$

$$
\begin{aligned}
& L:=\text { multiplication by the Kähler form } \mathrm{i} \cdot \omega=\mathrm{i} \cdot \sum_{n>0 ; a} n \cdot \varepsilon^{a}(n) \wedge \varepsilon^{a}(-n) \\
& \Lambda:=L^{*} ; \\
& H:=(p-q) \text { on } \mathscr{C}^{-p, q} \text {. }
\end{aligned}
$$

They satisfy the $\mathfrak{s l}_{2}$ commutation relations

(iv) $[\Lambda, L]=H, \quad[H, \Lambda]=2 \Lambda, \quad[H, L]=-2 L$,

and thus generate an action of $\mathfrak{s l}_{2}$ on $\mathscr{C}^{*, *}$. Notice that $\Lambda$ annihilates $\overline{\mathfrak{g}_{+}}$, and $\mathscr{C}^{1,1}=\overline{\mathfrak{g}_{+}} \oplus\left(\mathfrak{g}_{+}\right)^{t}$ is isotypical to the standard 2-dimensional representation of $\mathfrak{s l}_{2}$. The action on $\mathscr{C}^{*, *}$ (which is the exterior algebra of $\mathscr{C}^{1,1}$ ) is, therefore, integrable. The actions of these operators on a vector $\bar{\phi} \oplus \psi \in \overline{\mathfrak{g}_{+}} \oplus\left(\mathfrak{g}_{+}\right)^{t}$, written in column form with $\bar{\phi}$ as the top entry, are explicitly given by the matrices

$$
L=\left(\begin{array}{cc}
0 & 0 \\
-\mathrm{i} \cdot * & 0
\end{array}\right), \quad \Lambda=\left(\begin{array}{cc}
0 & \mathrm{i} \cdot * \\
0 & 0
\end{array}\right), \quad H=\left(\begin{array}{cc}
1 & 0 \\
0 & -1
\end{array}\right)
$$

We can conclude from here that

(v) $\exp \left(\frac{\pi}{2}(\Lambda-L)\right)=i^{|p+q|} *$.

Because the Kähler form is closed, we also have

(vi) $\left[L, D_{\text {vecz }}\right]=\left[L, \bar{\partial}_{\vec{z}}\right]=0$,

whereas, by considering the degrees,

$\left(\mathrm{vi}^{\prime}\right)\left[H, D_{\vec{z}}\right]=D_{\vec{z}},\left[H, \bar{\partial}_{\vec{z}}\right]=\bar{\partial}_{\vec{z}}$.

\section{Corollary B.1.6. ("The Kähler Identities").}

$$
D_{\vec{z}}^{*}=\mathrm{i} \cdot\left[\Lambda, \bar{\partial}_{\vec{z}}\right] \text {, and } \bar{\partial}_{\vec{z}}^{*}=(-\mathrm{i}) \cdot\left[\Lambda, D_{\vec{z}}\right] \text {. }
$$

Proof. The commutation action of $L, \Lambda$ and $H$ on $D_{\vec{z}}$ (resp. $\bar{\partial}_{\vec{z}}$ ) generates a space of operators on $\mathscr{C}^{*, *}$. Because of (vi) and $\left(\mathrm{vi}^{\prime}\right)$, this must be isomorphic to the 2 dimensional representation of $\mathfrak{s l}_{2}$ : the latter is the only integrable $\mathfrak{s I}_{2}$-representation 
generated by an $L$-invariant vector of $H$-weight 1 . But, in the 2-dimensional representation, the $L$-invariant vectors $v$ satisfy

$$
\exp \left(\frac{\pi}{2}(\Lambda-L)\right)(v)=\Lambda(v)
$$

Using (v) and (ii), and observing that $D_{\vec{z}}$ decreases $p$ by 1 , while $\bar{\partial}_{\vec{z}}$ increases $q$ by 1 , we obtain the Kähler identities.

Alternatively, but more painfully, one can verify the Kähler identities directly from the formulas for $D_{\vec{z}}$ and $\bar{\partial}_{\vec{z}}$.

We can finally prove Nakano's identity. Namely,

$$
\begin{aligned}
\square & =\left[D_{\vec{z}}, D_{\vec{z}}^{*}\right]=\mathrm{i} \cdot\left[D_{\vec{z}},\left[\Lambda, \bar{\partial}_{\vec{z}}\right]\right]=\mathrm{i} \cdot\left[\left[D_{\vec{z}}, \Lambda\right], \bar{\partial}_{\vec{z}}\right]+\mathrm{i} \cdot\left[\Lambda,\left[D_{\vec{z}}, \bar{\partial}_{\vec{z}}\right]\right] \\
& =\left[\bar{\partial}_{\vec{z}}^{*}, \bar{\partial}_{\vec{z}}\right]+\mathrm{i} \cdot\left[\Lambda,\left[D_{\vec{z}}, \bar{\partial}_{\vec{z}}\right]\right]=\bar{\square}+\mathrm{i} \cdot\left[\Lambda,\left[D_{\vec{z}}, \bar{\partial}_{\vec{z}}\right]\right],
\end{aligned}
$$

and Nakano's identity follows from the following two relations:

(vii) $\left[D_{\vec{z}}, \bar{\partial}_{\vec{z}}\right]=(h+2 c) \cdot \omega+\Theta_{\vec{V}(\vec{z})}$, and

(viii) $\left[\mathrm{i} \cdot \Lambda, \Theta_{\vec{V}(\vec{z})}\right]=-T_{\vec{V}(\vec{z})}^{\Lambda}$ on $\mathscr{C}^{0, q}$,

where, as in (2.4.2),

$$
\begin{aligned}
\Theta_{\vec{V}(\vec{z})}:= & \sum_{p, q>0}\left\{\left[\theta_{\vec{z}}\left(\xi_{a}(-p)\right), \theta_{\vec{z}}\left(\xi_{b}(q)\right)\right]\right. \\
& \left.-\theta_{\vec{z}}\left(\left[\xi_{a}, \xi_{b}\right](q-p)\right)\right\} \otimes \varepsilon^{a}(p) \wedge \varepsilon^{b}(-q)
\end{aligned}
$$

is the hermitian curvature of the holomorphic vector bundle $\vec{V}(\vec{z})$ over the flag variety $X=L G / G$. (viii) is a direct consequence of the relations $\left[\Lambda, \varepsilon^{a}(n)\right]=$ $-\mathrm{i} \cdot \varepsilon^{a}(-n)^{*},\left[\Lambda, \varepsilon^{a}(-n)\right]=-\mathrm{i} \cdot \varepsilon^{a}(n)^{*}$, and the definition of $T_{\vec{V}}^{\Lambda}$. We shall justify (vii) in a moment, but note that its right-hand side is the curvature of $\mathscr{L}^{\otimes(h+2 c)} \otimes \vec{V}(\vec{z})$. Geometrically speaking, it follows that we are studying sections of $\mathscr{L}^{\otimes(h+2 c)} \otimes \vec{V}(\vec{z}) \otimes \mathbf{K}_{X}$. Algebraically, we know we are working at high $h$. The shift $\mathscr{L}^{\otimes(-2 c)}$ in the level represents the canonical bundle of $X$.

Relation (B.1.7) now becomes, on $\mathscr{C}^{0, q}$,

$$
\square=\bar{\square}+\mathrm{i} \cdot(h+2 c) \cdot[\Lambda,(-\mathrm{i}) \cdot L]+\mathrm{i} \cdot\left[\Lambda, \Theta_{\vec{V}}\right]=\bar{\square}-(h+2 c) \cdot q-T_{\vec{V}}^{\Lambda},
$$

which is Nakano's Identity (2.4.7).

Finally, let us prove (vii). We go from $\mathscr{C}^{*, *}$ to the larger space (B.1.5), on which $\bar{\partial}_{\vec{z}}$ extends (cf. Remark B.1.4) to the differential

$$
\vec{\partial}_{\vec{z}}^{\#}:=\sum_{m \geqq 0 ; a}\left(R\left(\xi_{a}(m)\right)+\operatorname{ad}_{\xi_{a}(m)}^{t}+\frac{1}{2} \operatorname{ad}_{\xi_{a}(m)}+\theta_{\bar{z}}\left(\xi_{a}(m)\right)\right) \cdot \varepsilon^{a}(-m)
$$

for the Lie algebra cohomology of $\mathfrak{g}_{\mathbb{C}[z]}$ with coefficients in $\operatorname{Hom}\left(\Lambda^{q}\left(\mathfrak{g}_{+}\right) ; \hat{\mathscr{H}} \otimes\right.$ $\vec{V}(\vec{z}))$. 
We first observe that $\Theta_{\vec{V}(\vec{z})}$ comes from the differences $\delta_{\vec{z}}=D_{\vec{z}}-D_{0}$ and $\bar{\delta}_{\vec{z}}^{\#}=$ $\bar{\partial}_{\vec{z}}^{\#}-\bar{\partial}_{\vec{z}}^{\#}$ :

$$
\begin{aligned}
{\left[\delta_{\vec{z}}, \bar{\delta}_{\vec{z}}^{\#}\right] } & =\left[\sum_{p>0 ; a} \theta_{\vec{z}}\left(\xi_{a}(-p)\right) \cdot \varepsilon^{a}(p), \sum_{q \geqq 0 ; b} \theta_{\vec{z}}\left(\xi_{b}(q)\right) \cdot \varepsilon^{b}(-q)\right] \\
& =\sum_{\substack{p>0, q \geqq 0 \\
a, b}}\left[\theta_{\vec{z}}\left(\xi_{a}(-p)\right), \theta_{\vec{z}}\left(\xi_{b}(q)\right)\right] \otimes \varepsilon^{a}(p) \wedge \varepsilon^{b}(-q), \\
{\left[\delta_{\vec{z}}, \bar{\partial}_{0}^{\#}\right] } & =\sum_{\substack{p>0, q \geqq 0 \\
a, b}} \theta_{\vec{z}}\left(\xi_{a}(-p)\right) \cdot\left[\varepsilon^{a}(p), \operatorname{ad}_{\xi_{b}(q)}\right] \wedge \varepsilon^{b}(-q) \\
& \left.=\sum_{p>q \geqq 0 ; a, b} \theta_{\vec{z}}\left(\left[\xi_{b}, \xi_{a}\right]\right)(q-p)\right) \cdot \varepsilon^{a}(p) \wedge \varepsilon^{b}(-q), \\
{\left[D_{0}, \bar{\delta}_{\vec{z}}^{\#}\right] } & =\sum_{p>0, q \geqq 0} \theta_{\vec{z}}\left(\xi_{b}(q)\right) \cdot \varepsilon^{a}(p) \wedge\left[\operatorname{ad}_{\xi_{a}(-p)}, \varepsilon^{b}(-q)\right] \\
& \left.=\sum_{q \geqq p>0 ; a, b} \theta_{\vec{z}}\left(\left[\xi_{b}, \xi_{a}\right]\right)(q-p)\right) \cdot \varepsilon^{a}(p) \wedge \varepsilon^{b}(-q),
\end{aligned}
$$

and adding up the three r.h.s. terms gives $\Theta_{\vec{V} \vec{z}}$.

Last, we must show that on $\mathscr{C}^{*, *}$

$$
\left[D_{0}, \bar{\partial}_{0}^{\#}\right]=(h+2 c) \cdot \omega \text {. }
$$

Note that $D_{0}$ and $\vec{\partial}_{0}^{\#}$ no longer involve an action on the factor $\vec{V}$; they are the Lie algebra differentials with coefficients in $\operatorname{Hom}\left(\Lambda^{q}\left(\mathfrak{g}_{\mathbb{C}[z]}\right) ; \hat{\mathscr{H}}\right)$, resp. in $\hat{\mathscr{H}} \otimes \Lambda^{p}\left(\overline{\mathfrak{g}_{+}}\right)$. Thus in particular, $D_{0}^{2}=\left(\bar{\partial}_{0}^{\#}\right)^{2}=0$.

Let ad denote the action of $L \mathfrak{g}_{\mathbb{C}}$ on the space of semi-infinite exterior forms $\operatorname{Hom}\left(\Lambda^{q}\left(\mathfrak{g}_{\mathbb{C}[z]}\right) ; \Lambda^{p}\left(\overline{\mathfrak{g}_{+}}\right)\right)$. It is a projective ${ }^{25}$ action of level $(-2 c)$. The space $\operatorname{Hom}\left(\Lambda^{q}\left(\mathfrak{g}_{\mathbb{C}[z]}\right) ; \hat{\mathscr{H}} \otimes \Lambda^{p}\left(\overline{\mathfrak{g}_{+}}\right)\right) \otimes \vec{V}$, equipped with the operator

$$
d:=\sum_{a ; n}\left(R\left(\xi_{a}(n)\right)+\frac{1}{2} \cdot \widetilde{\operatorname{ad}}_{\xi_{a}(n)}\right) \varepsilon^{a}(-n),
$$

can be regarded as (a completion of) the complex of semi-infinite cohomology of $L \mathfrak{g}_{\mathbb{C}}$ with coefficients in $\hat{\mathscr{H}}$ (cf. $[\mathrm{Fe}],[\mathrm{F}-\mathrm{G}-\mathrm{Z}]$ ) (tensored with the irrelevant factor $\vec{V})$. The operators $D_{0}$ and $\bar{\partial}_{0}^{\#}$ add up to $d$; in addition, we note that $\left[\bar{\partial}_{0}, D_{0}\right]=d^{2}$. Consequently, (B.1.9) is equivalent to

$$
d^{2}=(h+2 c) \cdot \omega
$$

but this identity is well-known ([Fe, F-G-Z]): the term $h \cdot \omega$ stems from the projective cocycle of $\hat{\mathscr{H}}$, while the contribution $2 c \cdot \omega$ comes from the corresponding cocycle of the ad action on semi-infinite forms.

\footnotetext{
25 The phase is pinned down by requiring that it should restrict to non-projective actions of $\mathfrak{g}_{\mathbb{C}[z]}$ and $\mathfrak{g}_{\mathbb{C}\left[z^{-1}\right]}$.
} 
B.2. The "new" Laplacian $\bar{\square}_{0, \text { new }}$. We now bring the argument of Chapter 2 to a close by proving Lemma 3.3.4. This we accomplish by a direct calculation of the Laplacian $\bar{\square}_{0 \text {, new }}$. This is not very illuminating; a closely related, but more tasteful calculation was done by Taubes [Ta], who determined the spectrum of a Dirac operator on the line bundle $\mathscr{L}^{\otimes h}$ over $L G$, restricted to a domain of the form $\mathscr{H} \otimes$ (spinors on $L \mathfrak{g}$ ) (ours is the Dirac operator $\bar{\partial}_{0}+\bar{\partial}_{0}^{*}$ on $L G / G$ ); this was the source of moral strength behind the following pages of formulae.

In the remainder of this section, only the "new" hermitian metric \|\|$_{\text {new }}$ of Sect. 3.3 will be considered: we thus work in the Hilbert space completion of the $\mathfrak{g}$-invariant vectors in $\mathscr{H} \otimes \vec{V} \otimes \Lambda^{*}\left(\mathfrak{g}_{+}^{t}\right)$, where, as before, $\mathfrak{g}_{+}=\bigoplus_{n=1}^{\infty} \mathfrak{g}(n)$, but now $\left\{\xi^{a}(-n)\right\}_{n>0}$ is an orthonormal basis of $\mathfrak{g}_{+} \cdot\left(\left\{\xi_{a}\right\}\right.$ is an orthonormal basis of $\mathfrak{g}$ for the basic inner product.) The unitary structures of $\mathscr{H}$ and $\vec{V}$ are unchanged.

\section{Notation:}

$$
\begin{aligned}
& R_{a, m}=\text { the action of } \xi_{a}(m) \text { on } \mathscr{H} ; \\
& \varepsilon^{a}(-n)=\text { exterior multiplication by } \xi^{a}(-n) \text { on } \Lambda^{*}\left(\mathfrak{g}_{+}^{t}\right) ; \\
& A_{a, m}=\text { the co-adjoint action of } \xi_{a}(m) \text { on }\left(L \mathfrak{g}_{\mathbb{C}}\right)^{t}, \text { truncated to }\left(\mathfrak{g}_{+}\right)^{t} \text {; that is, } \\
& A_{a, m}\left(\xi^{b}(-n)\right)=\left\{\begin{array}{l}
{\left[\operatorname{ad}_{\zeta_{a}}^{t}\left(\xi^{b}\right)\right](m-n), \text { if } m-n<0 ;} \\
0, \text { otherwise. }
\end{array}\right.
\end{aligned}
$$

We denote the multilinear extension of $A_{a, m}$ to $\Lambda^{*}\left(\left(\mathfrak{g}_{+}\right)^{t}\right)$ by the same symbol $A_{a, m}$,

$$
L_{a, m}:=R_{a, m}+A_{a, m} .
$$

Notice that $R_{a, m}{ }^{*}=-R_{a,-m}$ and $A_{a, m}{ }^{*}=-A_{a,-m}$. For two indices $a$ and $b$ of the basis of $\mathfrak{g}$, we also let

$$
R_{[a, b], n}:=\text { the action of }\left[\xi_{a}, \xi_{b}\right](n) \text { on } \mathscr{H} .
$$

In the same vein we shall use the notations $\varepsilon^{[a, b]}(-n)$ and $A_{[a, b], n}$. We thus have

$$
\left[A_{a, m}, \varepsilon^{b}(-n)\right]=\left\{\begin{array}{l}
\varepsilon^{[a, b]}(m-n), \text { if } m-n<0 \\
0, \text { otherwise }
\end{array}\right.
$$

Let also

$$
\begin{aligned}
\Delta_{W} & :=\text { the Casimir operator }-\frac{1}{2} \sum_{b} \xi_{b}^{2} \text { acting on a representation } W \text { of } \mathfrak{g}, \\
c_{0} & :=\text { the eigenvalue of the Casimir on the space of zero-energy states of } \mathscr{H}, \\
E & :=\text { the sign-adjusted (i.e. positive) energy operator on } \mathscr{H} \otimes \Lambda^{*}\left(\left(\mathfrak{g}_{+}\right)^{t}\right) .
\end{aligned}
$$


We shall also write $\bar{\partial}, \bar{\partial}^{*}$ and $\bar{\square}$ for the operators $\bar{\partial}_{0}, \bar{\partial}_{0 \text {, new }}^{*}$ and $\bar{\square}_{0 \text {, new }}=\left[\bar{\partial}, \bar{\partial}^{*}\right]$. Then,

$$
\begin{aligned}
\bar{\partial} & =\sum_{n=1}^{\infty} \varepsilon^{b}(-n) \cdot\left(R_{b, n}+\frac{1}{2} A_{b, n}\right), \\
\bar{\partial}^{*} & =-\sum_{n=1}^{\infty}\left(R_{b,-n}+\frac{1}{2} A_{b,-n}\right) \cdot \varepsilon^{b}(-n)^{*} .
\end{aligned}
$$

(Summation over repeated Lie algebra indices is implied here and in all that follows.)

As can be seen from (B.2.1), $\bar{\partial}$ and $\bar{\partial}^{*}$ act trivially on the factor $\vec{V} \cong V_{1} \otimes \cdots \otimes$ $V_{n}$. The only role of $\vec{V}$ is to select the $G$-isotypical components of $\mathscr{H} \otimes \Lambda^{*}\left(\left(\mathfrak{g}_{+}\right)^{t}\right)$ which "contribute" to the domain $\mathscr{C}^{q}=\left[\mathscr{H} \otimes \vec{V}(0) \otimes \Lambda^{q}\left(\mathrm{~g}_{+}^{t}\right)\right]^{g}$.

\section{Proposition (B.2.2).}

$$
\bar{\square}=(h+c) \cdot E+c_{0}+\frac{1}{2} \cdot \sum_{b}\left(R_{b, 0}+A_{b, 0}\right)^{2}=(h+c) \cdot E+c_{0}-\Delta_{\vec{V}} .
$$

The first identity holds on all of $\mathscr{H} \otimes \vec{V} \otimes \Lambda^{*}\left(\left(\mathfrak{g}_{+}\right)^{t}\right)$; the second only holds on $\mathfrak{g}$-invariant forms.

Proof. We first check (B.2.2) on zero-forms. For $\psi \in \mathscr{H} \otimes \vec{V}$, we have

$$
\begin{aligned}
\bar{\square}(\psi) & =\left[\bar{\partial}, \bar{\partial}^{*}\right](\psi)=\bar{\partial}^{*} \bar{\partial} \psi \\
& =\sum_{p=1}^{\infty} \xi_{a}(-p) \xi_{a}(p) \psi \\
& =(h+c) \cdot E(\psi)+c_{0} \cdot \psi-\Delta_{\mathscr{H}}(\psi) .
\end{aligned}
$$

However, on zero-forms, $\Delta_{\mathscr{H}}=-\frac{1}{2} \cdot \sum_{b}\left(R_{b, 0}+A_{b, 0}\right)^{2}$, verifying (B.2.2). To settle the general case, we need to show that the operator $\bar{\square}-(h+c) \cdot E-c_{0}-$ $\frac{1}{2} \cdot \sum_{b} L_{b, 0}^{2}$ commutes with all exterior multiplications $\varepsilon^{a}(-m)$. Noting that $\left[E, \varepsilon^{a}(-m)\right]=m \cdot \varepsilon^{a}(-m)$ and that

$$
\begin{aligned}
{\left[\frac{1}{2} \cdot \sum_{b} L_{b, 0}^{2}, \varepsilon^{a}(-m)\right] } & =\frac{1}{2} \cdot \sum_{b}\left(\varepsilon^{[b, a]}(-m) \cdot L_{b, 0}+L_{b, 0} \varepsilon^{[b, a]}(-m)\right) \\
& =\frac{1}{2} \cdot \sum_{b} \varepsilon^{[b,[b, a]]}(-m)+\varepsilon^{[b, a]}(-m) \cdot L_{b, 0} \\
& =-c \cdot \varepsilon^{a}(-m)+\varepsilon^{b}(-m) \cdot L_{[a, b], 0},
\end{aligned}
$$

it suffices, in order to prove the proposition, to verify the following identity:

$\left[\bar{\square}, \varepsilon^{a}(-m)\right]=(h+c) \cdot m \cdot \varepsilon^{a}(-m)-c \cdot \varepsilon^{a}(-m)+\sum_{b} \varepsilon^{b}(-m) \cdot L_{[a, b], 0}$.

Proof of Identity (B.2.4). We have

$$
\left[\left[\bar{\partial}, \bar{\partial}^{*}\right], \varepsilon^{a}(-m)\right]=\left[\bar{\partial},\left[\bar{\partial}^{*}, \varepsilon^{a}(-m)\right]\right]+\left[\bar{\partial}^{*},\left[\bar{\partial}, \varepsilon^{a}(-m)\right]\right] .
$$


Now

$$
\left[\bar{\partial}, \varepsilon^{a}(-m)\right]=\frac{1}{2} \sum_{n=1}^{m-1} \varepsilon^{b}(-n) \wedge \varepsilon^{[b, a]}(n-m) .
$$

Noting the "classical" relations $\left[\bar{\partial}, \varepsilon^{a}(-m)^{*}\right]=L_{a, m},\left[\bar{\partial}^{*}, \varepsilon^{a}(-m)\right]=L_{a, m}^{*}=L_{a,-m}$, we get:

$$
\begin{aligned}
& {\left[\bar{\partial}^{*},\left[\bar{\partial}, \varepsilon^{a}(-m)\right]\right]=\frac{1}{2} \sum_{n=1}^{m-1}\left[\bar{\partial}^{*}, \varepsilon^{b}(-n) \wedge \varepsilon^{[b, a]}(n-m)\right]} \\
& =-\frac{1}{2} \sum_{n=1}^{m-1} L_{b,-n} \cdot \varepsilon^{[b, a]}(n-m)+\frac{1}{2} \sum_{n=1}^{m-1} \varepsilon^{b}(-n) \cdot L_{[b, a], n-m} \\
& =-\frac{1}{2} \sum_{n=1}^{m-1} \varepsilon^{[b,[b, a]]}(-m)-\frac{1}{2} \sum_{n=1}^{m-1} \varepsilon^{[b, a]}(n-m) \cdot L_{b,-n} \\
& +\frac{1}{2} \sum_{n=1}^{m-1} \varepsilon^{b}(-n) \cdot L_{[b, a], n-m} \\
& =c \cdot(m-1) \cdot \varepsilon^{a}(-m)-\sum_{n=1}^{m-1} \varepsilon^{b}(-n) \cdot L_{[a, b], n-m} \\
& {\left[\bar{\partial},\left[\bar{\partial}^{*}, \varepsilon^{a}(-m)\right]\right]=\left[\bar{\partial}, L_{a, m}^{*}\right]=\left[L_{a,-m}, \bar{\partial}\right]=\left[R_{a,-m}, \bar{\partial}\right]+\left[A_{a,-m}, \bar{\partial}\right]} \\
& =\sum_{n=1}^{\infty} \varepsilon^{b}(-n) \cdot\left[R_{a,-m}, R_{b, n}\right]+\sum_{n=1}^{\infty} \varepsilon^{[a, b]}(-n-m) \cdot R_{b, n} \\
& +\frac{1}{2} \sum_{n=1}^{\infty} \varepsilon^{[a, b]}(-n-m) \cdot A_{b, n}+\frac{1}{2} \sum_{n=1}^{\infty} \varepsilon^{b}(-n) \cdot\left[A_{a,-m}, A_{b, n}\right] \\
& =h \cdot m \cdot \varepsilon^{a}(-m)+\sum_{n=1}^{\infty} \varepsilon^{b}(-n) \cdot R_{[a, b], n-m}-\sum_{n=1}^{\infty} \varepsilon^{b}(-n-m) \cdot R_{[a, b], n} \\
& +\frac{1}{2} \sum_{n=1}^{\infty} \varepsilon^{b}(-n) \cdot A_{[a, b], n-m}-\frac{1}{2} \sum_{n=1}^{\infty} \varepsilon^{b}(-n-m) \cdot A_{[a, b], n} \\
& +\frac{1}{2} \sum_{n=1}^{\infty} \varepsilon^{b}(-n) \cdot\left(\left[A_{a,-m}, A_{b, n}\right]-A_{[a, b], n-m}\right) \\
& =h \cdot m \cdot \varepsilon^{a}(-m)+\sum_{n=1}^{m} \varepsilon^{b}(-n) \cdot\left(R_{[a, b], n-m}+\frac{1}{2} A_{[a, b], n-m}\right) \\
& +\frac{1}{2} \sum_{n=1}^{\infty} \varepsilon^{b}(-n) \cdot\left(\left[A_{a,-m}, A_{b, n}\right]-A_{[a, b], n-m}\right) \text {. }
\end{aligned}
$$

By direct inspection,

$$
\left(\left[A_{a,-m}, A_{b, a}\right]-A_{[a, b], n-m}\right)\left(\varepsilon^{d}(-p)\right)=\left\{\begin{array}{l}
0, \text { if } p>n \text { or } p \leqq n-m \\
-\varepsilon^{[a,[b, d]]}(n-m-p), \text { otherwise }
\end{array}\right.
$$


whence

$$
\begin{aligned}
{\left[A_{a,-m}, A_{b, n}\right]-A_{[a, b], n-m}=-\sum_{\substack{p=n-m+1 \\
p \geqq 0}}^{n} \varepsilon^{[a,[b, d]]}(n-m-p) \cdot \varepsilon^{d}(-p)^{*} } \\
\frac{1}{2} \sum_{n=1}^{\infty} \varepsilon^{b}(-n) \cdot\left(\left[A_{a,-m}, A_{b, n}\right]-A_{[a, b], n-m}\right) \\
=-\frac{1}{2} \cdot \sum_{n=1}^{\infty} \sum_{p=n-m+1}^{n} \varepsilon^{b}(-n) \wedge \varepsilon^{[a,[b, d]]}(n-m-p) \cdot \varepsilon^{d}(-p)^{*} \\
=\frac{1}{2} \cdot \sum_{p=1}^{\infty} \sum_{n=p}^{p+m-1} \varepsilon^{b}(-n) \wedge \varepsilon^{[a,[b, d]]}(n-m-p) \cdot \varepsilon^{d}(-p)^{*} \\
=\frac{1}{2} \cdot \sum_{p=1}^{\infty} \sum_{n=p}^{p+m-1} \varepsilon^{[b, d]}(-n) \wedge \varepsilon^{[a, b]}(n-m-p) \cdot \varepsilon^{d}(-p)^{*} \\
=\frac{1}{2} \cdot \sum_{p=1}^{\infty} \sum_{n=p}^{p+m-1} \varepsilon^{[a, b]}(n-m-p) \wedge \varepsilon^{[b, d]}(-n) \cdot \varepsilon^{d}(-p)^{*} \\
=-\frac{1}{2} \cdot \sum_{p=1}^{\infty} \sum_{n=0}^{m-1} \varepsilon^{[a, b]}(n-m) \wedge \varepsilon^{[b, d]}(-n-p) \cdot \varepsilon^{d}(-p)^{*} \\
=-\frac{1}{2} \cdot \sum_{n=0}^{m-1} \varepsilon^{[a, b]}(n-m) \cdot A_{b,-n}=-\frac{1}{2} \cdot \sum_{n=1}^{m} \varepsilon^{[a, b]}(-n) \cdot A_{b, n-m} \\
=\frac{1}{2} \cdot \sum_{n=1}^{m} \varepsilon^{b}(-n) \cdot A_{[a, b], n-m} \cdot
\end{aligned}
$$

We therefore have

$$
\begin{aligned}
{\left[\bar{\partial},\left[\bar{\partial}^{*}, \varepsilon^{a}(-m)\right]\right]=} & h \cdot m \cdot \varepsilon^{a}(-m)+\sum_{n=1}^{m} \varepsilon^{b}(-n) \\
& \cdot\left(R_{[a, b], n-m}+\frac{1}{2} \cdot A_{[a, b], n-m}+\frac{1}{2} \cdot A_{[a, b], n-m}\right) \\
= & h \cdot m \cdot \varepsilon^{a}(-m)+\sum_{n=1}^{m} \varepsilon^{b}(-n) \cdot L_{[a, b], n-m},
\end{aligned}
$$

and from (B.2.5) and (B.2.6) we obtain

$$
\left[\bar{\square}, \varepsilon^{a}(-m)\right]=(h+c) \cdot m \cdot \varepsilon^{a}(-m)-c \cdot \varepsilon^{a}(-m)+\varepsilon^{b}(-m) \cdot L_{[a, b], 0},
$$

which concludes the proof of the identity B.2.4 and therefore of Proposition B.2.2.

\section{Appendix C. Two Useful Inequalities}

This appendix is devoted to the proof of Proposition 2.5.5 of Chap. 2:

\section{Proposition (Chap. 2, 2.5.5).}

(i) $\sum_{a} \theta_{k}\left(\xi_{a}\right) \otimes \mathrm{ad}_{\xi_{a}}^{t} \leqq\left(\lambda_{k}+2 \rho\right) \cdot \alpha_{\max }$ on $V_{k} \otimes\left(\mathfrak{g}_{\mathbb{C}}\right)^{t}$;

(ii) For every $\varepsilon>0$, we have $\sum_{k} H_{z_{k}}^{-1}<1+\varepsilon$ if the points $z_{k} \in \Delta$ are sufficiently far apart in the hyperbolic metric. 
Proof of (i). We show, more generally, that for two representations $E$ and $F$ of $\mathrm{g}$, with highest weights $\lambda_{E}$ and $\lambda_{F}$, we have

$$
\sum_{a} \xi_{a} \otimes \xi_{a} \leqq-w_{\max }\left(\lambda_{F}\right) \cdot\left(\lambda_{E}+2 \rho\right),
$$

where $w_{\max }$ is the longest element of the Weyl group. Note that $-w_{\max }\left(\lambda_{F}\right)$ is the highest weight of $F^{t}$. With $E=V_{k}$ and $F=\left(\mathfrak{g}_{\mathbb{C}}\right)^{t}$, we obtain, as desired,

$$
\sum_{a} \xi_{a} \otimes \xi_{a} \leqq \alpha_{\max } \cdot\left(\lambda_{k}+2 \rho\right) .
$$

Note. Relation C.1 is not the best possible estimate. Rahter, the best bound is

$$
-w_{\max }\left(\lambda_{F}\right) \cdot \lambda_{E}+\left(\lambda_{E}+\lambda_{F}\right) \cdot \rho-\sup _{w \in W}\left(\left(\lambda_{E}+w_{\max }\left(\lambda_{F}\right)\right) \cdot w(\rho)\right)
$$

from which we get C.1 by setting $w=1$.

Denote by $\Delta_{V}$ the Casimir operator of $\mathfrak{g}$ acting on a representation $V$. Then,

$$
\begin{aligned}
\sum_{a} \xi_{a} \otimes \xi_{a} & =-\Delta_{E \otimes F}+\Delta_{E} \otimes 1+1 \otimes \Delta_{F} \\
& =-\Delta_{E \otimes F}+\frac{1}{2}\left(\lambda_{E}+\rho\right)^{2}+\frac{1}{2}\left(\lambda_{F}+\rho\right)^{2}-\rho^{2} .
\end{aligned}
$$

Substituting this in C.1 reduces the latter to

$$
\Delta_{E \otimes F} \leqq \frac{1}{2} \cdot\left(\lambda_{E}+w_{\max }\left(\lambda_{F}\right)+\rho\right)^{2}-\frac{1}{2} \rho^{2} .
$$

In other words, for the highest weight $\lambda$ of any sub-representation of $E \otimes F$, we need to have

$$
\left(\lambda_{E}+w_{\max }\left(\lambda_{F}\right)+\rho\right)^{2} \leqq(\lambda+\rho)^{2},
$$

Let $C$ be the positive Weyl chamber in $\mathrm{t}$, and denote by $K$ the cone dual to $C$. It is spanned by the positive roots in $\mathrm{t}$. For an element $\alpha \in C$, let $C W(\alpha)$ denote the convex hull of the Weyl transforms of $\alpha$. It is the intersection of the Weyl transforms of the shifted cones $\alpha-K$.

Now the representation $V_{\lambda}$ of highest weight $\lambda$ occurs in $E \otimes F$ iff $E$ occurs in $V_{\lambda} \otimes F^{t}$. But, since all the highest weights in the tensor product $V_{\lambda} \otimes F^{t}$ fall within the shifted convex hull $-w_{\max }\left(\lambda_{F}\right)+C W(\lambda)$, the latter can only happen if

$$
\lambda_{E}+w_{\max }\left(\lambda_{F}\right) \in C W(\lambda) \text {. }
$$

But then, as we shall explain, it follows that

$$
\left(\lambda_{E}+w_{\max }\left(\lambda_{F}\right)\right)^{2} \leqq \lambda^{2}, \text { and }\left(\lambda_{E}+w_{\max }\left(\lambda_{F}\right)\right) \cdot \rho \leqq \lambda \cdot \rho,
$$

whence we deduce (C.2). The first inequality is obvious, the second follows from the fact that $\rho$ is dominant and $\left(\lambda-\lambda_{E}-w_{\max }\left(\lambda_{F}\right)\right) \in K$.

Proof of (ii). Given a positive integer $N$, denote by $S_{k}$ the span of eigenvectors corresponding to the eigenvalues $1,1 / 2, \ldots, 1 / N$ of $H_{z_{k}}^{-1}$. Denoting by $S_{0}$ the orthocomplement of the sum of the spaces $S_{k}$, we have a decomposition

$$
\mathfrak{g}_{+}=S_{0} \oplus S_{1} \oplus \cdots \oplus S_{n} .
$$


(Vectors in different $S_{k}$ 's are linearly independent, since their analytic continuations outside the unit disk have poles at the distinct points $\left(\overline{z_{k}}\right)^{-1}$.) Somewhat abusively, $\mathfrak{g}_{+}$stands for its own Hilbert space completion. I claim that, in the limit when the points move far apart, the decomposition C. 4 becomes nearly orthogonal. More precisely:

Claim. Given any $\delta>0$, the inner product of any two unit vectors in different $S_{k}$ 's is less than $\delta$, if the points are far apart in the hyperbolic metric.

Granting this for a moment, pick $v=v_{0} \oplus \cdots \oplus v_{n} \in \mathfrak{g}_{+}$. We have

$$
\left\|\sum_{k=1}^{n} H_{z_{k}}^{-1}(v)\right\|=\left\|\sum_{k=1 ; j=0}^{n} H_{z_{k}}^{-1}\left(v_{j}\right)\right\| \leqq\left\|\sum_{k=1}^{n} H_{z_{k}}^{-1}\left(v_{k}\right)\right\|+\left\|\sum_{\substack{k=1 ; j=0 \\ j \neq k}}^{n} H_{z_{k}}^{-1}\left(v_{j}\right)\right\| \text {. }
$$

But we also have

$$
\left\|\sum_{k=1}^{n} H_{z_{k}}^{-1}\left(v_{k}\right)\right\|<\left(1+\delta^{\prime}\right) \cdot\left(\sum_{k}\left\|v_{k}\right\|^{2}\right)^{1 / 2}=\left(1+\delta^{\prime}\right) \cdot\|v\|
$$

for some $\delta^{\prime}$ depending on $\delta$, because $H_{z_{k}}^{-1}\left(v_{k}\right) \in S_{k},\left\|H_{z_{k}}^{-1}\left(v_{k}\right)\right\| \leqq\left\|v_{k}\right\|$, and the mixed inner products are negligible, by our claim. In addition,

$$
\left\|\sum_{\substack{k=1, j=0 \\ j \neq k}}^{n} H_{z_{k}}^{-1}\left(v_{j}\right)\right\|<\left(\frac{n}{N}+\delta \cdot n\right) \sum_{j}\left\|v_{j}\right\| \leqq\left(\frac{n}{N}+\delta \cdot n\right) \sqrt{n} \cdot\|v\|,
$$

because

$$
\left\|H_{z_{k}}^{-1}\left(v_{j}\right)\right\|<\delta \cdot\left\|v_{J}\right\|+\frac{1}{N} \cdot\left\|v_{j}\right\|
$$

as can be seen by decomposing $v_{j}$ into its $S_{k}$-component and complementary component. This proves (ii), if $N$ is chosen large enough and $\delta^{\prime}$ small enough.

Proof of the Claim. Because the $S_{k}$ 's are finite-dimensional, and because of the $S L_{2}(\mathbb{R})$-invariance, we only need to show that two given eigenvectors for $H_{0}^{-1}$ and $H_{z_{k}}^{-1}$ become orthogonal when $z_{k}$ approaches the boundary. In geometric terms, this means that the function $z^{p} / \sqrt{p}$ is nearly orthogonal, in the inner product 2.1 .1 , to the transform $\phi_{q}$ of $z^{q} / \sqrt{q}$ under an element $\phi$ of $S L_{2}(\mathbb{R})$ taking 0 to $z_{k}$. But $\phi_{q}$, restricted to the unit circle, is a function with values in the unit circle in $\mathbb{C}$, nearly equal to 1 everywhere except in a small neighbourhood of $z_{k}$, where it quickly winds $q$ times around 0 . Its $L^{2}$ inner product with a positive Fourier mode is, therefore, vanishingly small in the limit when $z_{k}$ approaches the boundary. It follows that

$$
2 \pi \mathrm{i} \cdot\left\langle\phi_{q} \mid z^{p} / \sqrt{p}\right\rangle=\oint \overline{\phi_{q}} \cdot \partial\left(z^{p} / \sqrt{p}\right)=\sqrt{p} \cdot \oint \overline{\phi_{q}} \cdot z^{p-1} d z \rightarrow 0 .
$$

\section{References}

[B] Bott, R.: Homogeneous vector Bundles. Ann. of Math. 66, 203-248 (1957)

[B2] Bott, R.: Letter to G. Segal, June 1993

[F] Faltings, G.: A Proof of the Verlinde Formula. Preprint, 1992

[F2] Faltings, G.: A proof of the Verlinde Formula. J. Alg. Geom. (1994) 
[Fe] Feigin, B.L.: The semi-infinite homology of Kac-Moody and Virasoro algebras. Russ. Math. Surv. 39 n. 2, 155-156 (1984)

[F-G-Z] Frenkel, B.I., Garland, H., Zuckerman, G.J.: Semi-infinite Cohomology and String Theory. Yale preprint, 1986

[Fr] Freed, D.: The Geometry of Loop Groups. J. Diff. Geom. 28, 223-276 (1988)

[F-F] Feigin, B.L., Fuchs, D.B.: Cohomology of some nilpotent subalgebras of the Virasoro and Kac-Moody Lie algebras. J.G.P 5, n. 2, 209-235 (1988)

[K] Kostant, B.: Lie Algebra Cohomology and the Generalized Borel-Weil Theorem. Ann. of Math. 74, 329-387 (1961)

[Ku] Kumar, S.: Demazure Character Formula in Arbitrary Kac-Moody Setting. Invent. Math. 89, 395-423 (1987)

[P-S] Pressley, A., Segal, G.: Loop Groups. New York: Oxford University Press, 1988

[RC-W] Rocha-Caridi, A., Wallach, N.: Projective Modules over Graded Lie Algebras. Math. Z. 180, 151-177 (1982)

[S] Segal, G.: The Definition of Conformal Field Theory. Unpublished manuscript

[S-S] Shiffmann, B., Sommese, A.J.: Vanishing Theorems on Complex Manifolds. Boston: Birkhäuser (1985)

[Ta] Taubes, C.: The Dirac Operator on a Loop Group. Unpublished notes

[T-K] Tsuchiya, A., Kanie, Y.: Vertex Operators in Conformal Field theory on $\mathbb{P}^{1}$ and Monodromy Representations of the Braid Group. In: Advanced Studies in Pure Mathematics 16, 297-372 (1988)

[T-Y] Tsuchiya, A., Ueno, K., Yamada, Y.: Conformal Field Theory on Universal Family of Stable Curves with Gauge Symmetries. In: Advanced Studies in Pure Mathematics 19, 459-566 (1989)

[V] Verlinde, E.: Fusion Rules and Modular Transformations in 2D Conformal Field Theory. Nucl. Phys. B300, 360--376 (1988)

Communicated by A. Jaffe 
